$13 / 2 / 8$
$6 / L A-7238-P R$

Progress Report

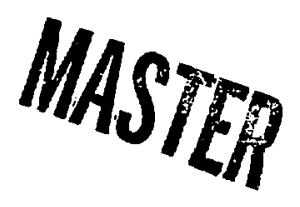

\title{
Medium-Energy Physics Program
}

\section{August 1-October 31, 1977}


The four most recent reports in this series, unclassified, are LA-6678-PR, LA-6819-PR, LA-6938-PR, and LA-7081-PR.

Primed in the United States of Ameritu. Available from National Technical Information Service

U.S. Department of Commetco

5285 Port Ruyil Resid

Microlkhe $\$ 3.00$

$\begin{array}{llllllllll}001-025 & 4.100 & 126-150 & 7.25 & 251-275 & 10.75 & 376-400 & 13.00 & 501-525 & 15.25 \\ 026-050 & 4.50 & 151-175 & 8.00 & 276.300 & 11.00 & 40 .-25 & 13.25 & 526-550 & 15.50 \\ 051-075 & 5.25 & 176-200 & 9.00 & 301-325 & 11.75 & 426-450 & 14.00 & 551-575 & 16.25 \\ 076-100 & 6.00 & 201.225 & 9.25 & 326-350 & 12.00 & 451-475 & 14.50 & 576-600 & 16.50 \\ 101-125 & 6.50 & 226-250 & 9.50 & 351-375 & 12.50 & 476-500 & 15.00 & 601-4 p & --1\end{array}$

1. Add 52,50 for each additional 100-page incremient from 601 pages up.

This report was prepared an an account of work sponwred by the United Stutes Govetmment. Neither the United Ststes nor the United States Department of Eneray, nor any of their employeer, nor any of their contractors, subeontractork or their employees, makes any warranty, expres or implied, or compleiene or utefulnes of any intomation, acouratus. product or procese diselosed or reyrements thet its use would not infringe privately owned richle. 


\section{CONTENTS}

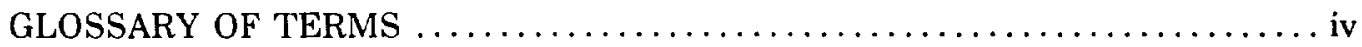

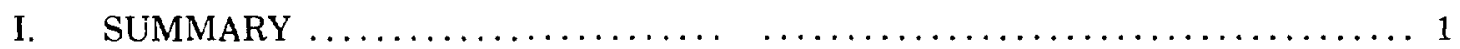

II. FACILITY OPERATION $\ldots \ldots \ldots \ldots \ldots \ldots \ldots \ldots \ldots \ldots \ldots \ldots \ldots \ldots \ldots \ldots \ldots \ldots \ldots \ldots$

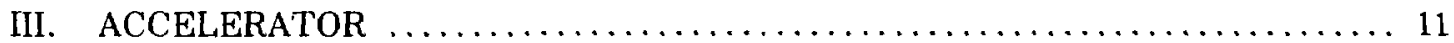

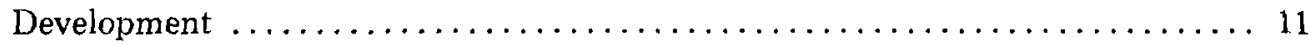

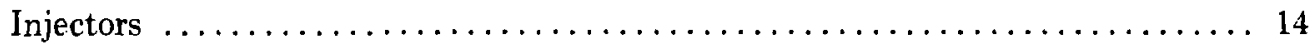

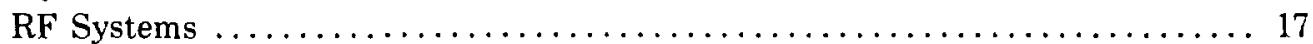

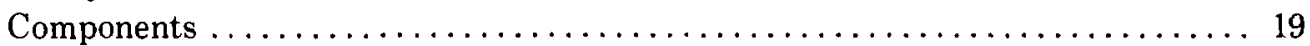

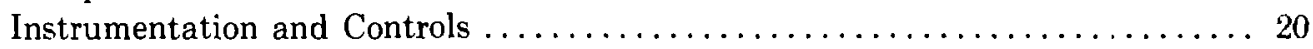

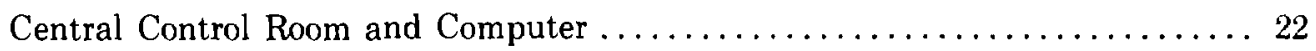

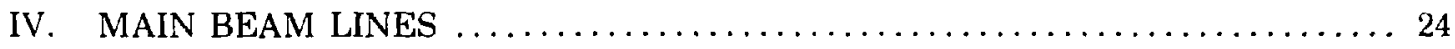

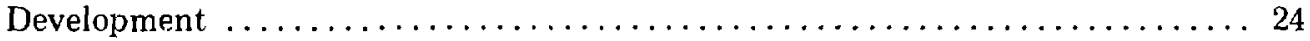

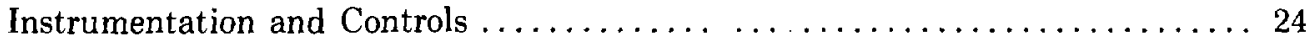

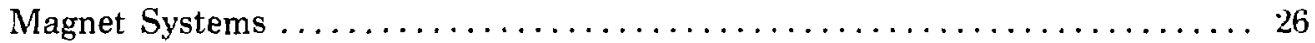

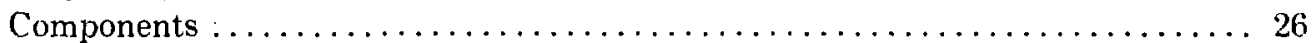

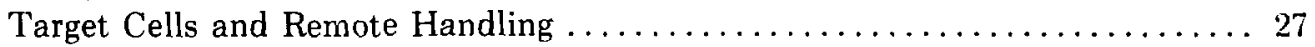

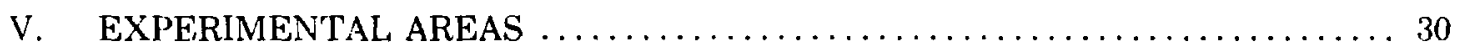

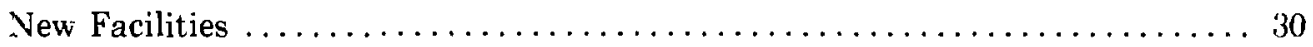

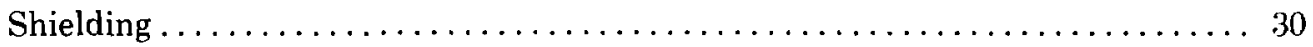

Secondary Beam Line Development $\ldots \ldots \ldots \ldots \ldots \ldots \ldots \ldots \ldots \ldots \ldots \ldots \ldots \ldots \ldots$

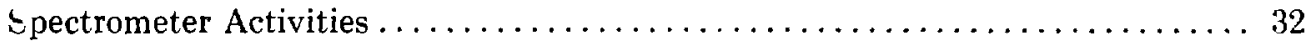

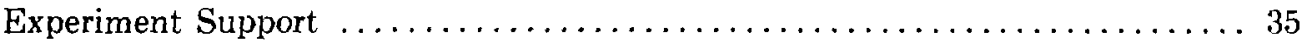

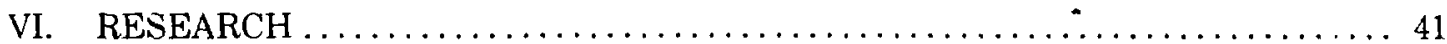

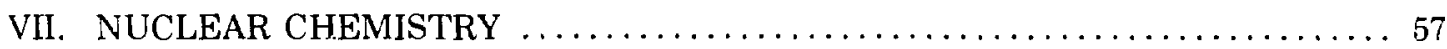

VIII. PRACTICAL APPLICATIONS OF LAMPF $\ldots \ldots \ldots \ldots \ldots \ldots \ldots \ldots \ldots \ldots \ldots$

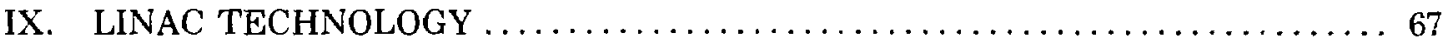

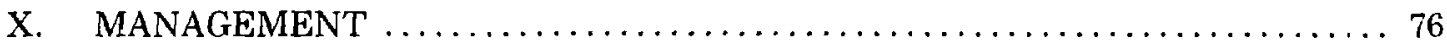

APPENDIX:

RESEARCH GUESTS AT LAMPF $\ldots \ldots \ldots \ldots \ldots \ldots \ldots \ldots \ldots \ldots \ldots \ldots$ 


\section{GLOSSARY OF TERMS USED IN THIS REPORT}

\begin{tabular}{|c|c|}
\hline $\mathrm{ADC}$ & - Analog Digital Converter \\
\hline ADS & - Analog Data System \\
\hline AMO & - Area Manager's Office \\
\hline $\mathrm{AP}$ & - Activation-Protect \\
\hline $\mathrm{APF}$ & - Alternating Phase-Focusing \\
\hline ASCII & $\begin{array}{l}\text { - American Standard Code for Infor- } \\
\text { mation Interchange }\end{array}$ \\
\hline bpi & - Bytes Per Inch \\
\hline BPM & - Beam-Position Monitor \\
\hline CCF & - LASL Central Computer Facility \\
\hline CCR & - Computer Control Room \\
\hline CCTV & - Closed-Circuit Television \\
\hline CIU & - Console-Interface Unit \\
\hline CPU & - Central Processing Unit \\
\hline CRT & - Cathode Ray Tube \\
\hline $\mathrm{cw}^{*}$ & - Continuous Wave \\
\hline C-W & - Cockcroft-Walton \\
\hline DACT & $\begin{array}{l}\text { - Data-Acquisition and Control Ter- } \\
\text { minal }\end{array}$ \\
\hline DPM & - Digital Panel Meter \\
\hline DVM & - Digital Volt Meter \\
\hline EFB & - Effective Field Boundary \\
\hline ETL & - Equipment Test Laboratory \\
\hline FET & - Field-Effect Transistor \\
\hline th & - Filament Hours \\
\hline FSI & - Final-State Interaction \\
\hline FWSS & - Fast-Wire-Scanner System \\
\hline hfs & - Hyperfine Structure \\
\hline IC & - Integrated Circuit \\
\hline $\mathrm{I} \& \mathrm{C}$ & - Instrumentation and Control \\
\hline ICR & - Injector Control Room \\
\hline IDS & - Information Display System \\
\hline IFA & - Interface Ainplifier \\
\hline $\mathrm{I} / \mathrm{O}$ & - Input/Output \\
\hline IPA & - Intermediate Power Amplifier \\
\hline ISIC & - Insertable-Strip Ion Chambers \\
\hline IVT: & - Induction Volt Regulator \\
\hline LAM & - Look-at-Me Interrupt \\
\hline LAMPF & $\begin{array}{l}\text { - Clinton P. Anderson Meson Physics } \\
\text { Facility }\end{array}$ \\
\hline LCF & - Localized Current Fields \\
\hline LED & - Light-Emittıng Diode \\
\hline LEEP & $\begin{array}{l}\text { - LAMPF Electronics and Equipment } \\
\text { Pool }\end{array}$ \\
\hline LET & - Linear-Energy Transfer \\
\hline LM & - Loss Monitor \\
\hline LTSS & - Livermore Time-Sharing System \\
\hline LUGI & - LAMPF Users Group, Inc. \\
\hline MBD & - Micropróbrammed Branch Driver \\
\hline
\end{tabular}

$\begin{array}{ll}\text { MG Set } & \text { - Motor-Generator-Set } \\ \text { m.i. } & \text { - Mineral Insulated } \\ \text { MPU } & \text { - Microprocessing Unit } \\ \text { MT } & \text { - Master Timer } \\ \text { MTBF } & \text { - Mean Time Between Failures } \\ \text { MWPC } & \text { - Multiwire Proportional Chamber } \\ \text { NCL } & \text { - Nuclear Chemistry Laboratory } \\ \text { NIM } & \text { - Nuclear Instrumentation Module } \\ \text { NMR } & \text { - Nuclear Magnetic Resonance } \\ \text { OER } & \text { - Oxygen Enhancement Ratio } \\ \text { pc } & \text { - Printed Circuit } \\ \text { PHA } & \text { - Pulse-Height Analyzer } \\ \text { PIGMI } & \text { - Pion Generator for Medical } \\ & \quad \text { Irradiations } \\ \text { PIP } & \text { - Proton Irradiation Port } \\ \text { PMT } & \text { - Photomultiplier Tube } \\ \text { RAM } & \text { - Random Access Memory } \\ \text { RBE } & \text { - Relative Biological Effectiveness } \\ \text { RICE } & \text { - Remote Information and Control } \\ & \quad \text { Equipment (remote data } \\ & \text { terminal) } \\ \text { RIU } & \text { - RICE Interface Unit } \\ \text { ROM } & \text { - Read-Only Memory } \\ \text { SCC } & \text { - Serial Crate Controller } \\ \text { SCR } & \text { - Silicon Control Rectifier } \\ \text { SSD } & \text { - Serial System Driver } \\ \text { SY } & \text { - Switchyard } \\ \text { TDC } & \text { - Time-to-Digital Converter } \\ \text { TDI } & \text { - Temperature Difference Integrator } \\ \text { TOF } & \text { - Time of Flight } \\ \text { TR } & \text { - Transition Region } \\ \text { VSWR } & \text { - Voltage Standing-Wave Ratio } \\ & \end{array}$

\section{Experimental Area}

Primary beam lines in experimental area:

Line A - Main Beam Line for Pion and Muon Channels

Line B - Neutron Beams and Nuclear Chemistry Facility

Line C - High-Resolution Proton Spectrometer

Line D - Weapons Neutron Research Facility

Experimental beams:

Beam Area A:

BSA - Beam Stop A

EPICS - Energetic Pion Channel and Spectrometers

LEP - Low-Energy Pion Channel

$P^{3}$ - High-Energy Pion Channel 
SMC - Stopped Muon Channel

TA-1 - Target A-1

TA-2 - Target A-2

TTA - Thin Target Area

Beam Area $B$ (Room BR):

$A B$ - Neutrons

AB - Nuclear Chemistry

EPB - External Proton Beam
Beam Area C:

$\mathrm{CCH}$ - Area $\mathrm{C}$ Control and Counting House

HRS - High-Resolution Proton Spectrometer

Beam Area A-East:

Biomedical Pion Channel

TA-5 - Target A-5

ISORAD - Isotope Production and Radiation Damage

Neutrino Area 


\title{
QUARTERLY REPORT ON THE MEDIUM-ENERGY PHYSICS PROGRAM
}

\author{
FOR THE PERIOD ENDING OCTOBER 31,1977
}

\section{SUMMARY}

\section{Facility Operation}

Beam was delivered to 27 experiments during the 21-day August run included in this quarter. The $\mathrm{H}^{+}$ current was increased from $225 \mu \mathrm{A}_{\mathrm{A}}$ to $300 \mu \hat{A}_{\mathrm{A}} \mathrm{d} u r$ ing the final seven shifts of proauction. Polarized $\mathrm{H}^{-}$beam $\left(\mathrm{P}^{-}\right)$was delivered for 15 days, unpolarized $\mathrm{H}^{-}$for the balance. Beam availability was $80 \%$ for $\mathrm{H}^{+}, 63 \%$ for $\mathrm{H}^{-}$, and $70 \%$ for $\mathrm{P}^{-}$. The accelerator was off and the facility power consumption minimized by all achievable means during the September budgetary shutdown.

\section{Accelerator}

The accelerator was back in operation for development for 24 days of October, part of the time in a new rf-low-duty mode, saving more than $1000 \mathrm{MWh}$ for this month. The $\mathrm{H}^{+}$beam emittance was reduced, and an $8 \mathrm{~mA}_{\mathrm{P}}, 625 \mu \mathrm{s}, 3.75 \mathrm{~Hz}$ beam was accelerated to $800 \mathrm{MeV}$ with good transnission.

Analysis of $\Delta t$ tuneup records has led to an appreciation of nonlinear effects of choice of peak beam current; $1-\mathrm{mA}_{\mathrm{P}}$ beams have produced the best tunes. A successful servo control of horizontal beam oscillations was demonstrated. Emittance growth and damping effects in the linac were measured.

A simulation code from CERN for the injector and drift-tube linac is being adapted to LAMPF. PARMILA and SIMALAC, the LAMPF design codes for the drift-tube and side-coupled linacs, are being coordinated and extended, for example, by inclusion of magnet multipole aberrations. A new ion source simulation code SNOW is in use.

The polarized $\mathrm{H}^{-}$source produced beams up to $3 \mathrm{nA}_{\mathrm{A}}$ for experimenters' use with an automatic spin-reversal cycle implemented. A laboratory, with $100-\mathrm{kV}$ test stand, is being refurbished and instrumented for ion source studies.

Modifications to the rf system were completed which provide a faster klystron turn-off to save power and permit selective blanking of transient error signals. Tank resonance system modifications and calibrations were completed through the linac. The phase subsystem modification and refurbishment was also completed on installed units. The $\mathrm{rf}$ sources were upgraded.

Realignment or measurement was done on several components from the $\mathrm{H}^{+}$prebuncher through Tank 14. Two new steering magnets were installed in the TR main line.

Multigain amplifiers were installed in six linac wire scanners. Eight new ceramic harp boards were installed, with fabrication techniques for siliconcarbide wires under study. A measurement was made of bias effects on the EM-2 emittance jaws.

Among other $\Delta t$ modifications, simulation hardware was installed.

The new master timer system was installed, providing low-duty rf gates and independent control of $\mathrm{H}^{+}, \mathrm{H}^{-}$and $\mathrm{P}^{-}$beam gates, and three calibrate gates.

A new disk system with PDP-11/34 interface control to the SEL-840 was implemented.

\section{Main Beam Lines}

A technique for emittance measurement on $\mathrm{H}^{+}$ beams in the switchyard was developed; the measurement uses six or seven SY wire scanners plus magnet calibration data. With this procedure, emittance parameters can be calculated from data taken with a standarcl production tune and with beam run at full duty. A new tune at A-5 was employed to increase the spot size in order to decrease possible thermal damage. 
A redesigned harp with commercial ceramic feedthroughs was installed. The guard ring system was connected to the Fast-Protect system. All haloremoving strippers in Line $\mathrm{X}$ were replaced with a new independent-jaw model.

Additional thermal protective instrumentation and interlocks were added to several areas. One such change is run-permit interlocking on the magnet cooling-water pumps. The AMO now has computer terminal access to status on many of these experimental area sytems.

Run-permit system changes included rebuilding of the interlock chassis for the $\mathrm{H}^{-}$beam areas and addition of time-out on restricted-access status if the door is left open on caves in Area A

Among the upgrading in progress on int mesnet systems is addition of a fault memory a ir a: play panel to power supplies. Assembly of the reconfigured indirectly-cooled target-cel, iriplet is proceeding. The WNR fast-kicker magnet is installed in Line A. Area A vacuum pumping speed has been increased.

New target wheels were installed at A-1, a target wheel chain-drive mechanism was installed at A-2, and a motor-driven actuator at A-5. A pyrolytic graphite target brazed to stainless-steel watercooling tubes is being built for A-5.

Extensive remote handling repairs were done at A-5 and A-6.

\section{Experimental Areas}

Construction design for "Package I" of the Area AEast cover and crane structure is complete. A fivemagnet spin-precession apparatus has been designed for EPB to permit compatible polarized beam operation of Line $\mathrm{C}$ and $\mathrm{EPB}$ with any proton spin orientation.

Shielding was added to EPICS and HRS. A heavily shielded cave and beam stop are being added at the end of EPB for the parity-violation experiment.

A method for accurate differential time-of-flight was developed and used on $\mathrm{P}^{3}$ to establish a $1-3 \%$ correction to the nominal channel momentum. A stopping beam of $8 \times 10^{8} \pi^{-}$per $\mathrm{g} / \mathrm{cm}^{2}$ of calcium at $300 \mu \mathrm{A}$ has been developed in SMC-East for $\pi$ capture $x$-ray experiments.
EPICS and HRS were used as scheduled for experimenters. In the two-month shutdown, a large scattering chamber was installed at EPICS, and the scattering chamber sliding seal was repaired at HRS. A new power supply was installed for the EPICS beam separator, and most of the EPICS and HRS delay-line chambers were replaced. Program development concentrated on improving data rate.

The beam optics design was completed for a lowresolution spectrometer, which is to become a second arm to HRS.

Eleven experiments received engineering support in this quarter. Two experiments scheduled for LEP, pions on hydrogen and deuterium (Exp. 96) and $\pi$-charge exchange (Exp. 181), in particular, required fabrication of major hardware items, including wire chamber assemblies. New direct-cooled coils were installed on the Ames $\mathrm{C}$ magnet, permitting reliable operation up to saturation. The inventory of vacuum hardware is being replenished and extended to shorten turn-around time for experimenters' fabrication.

Two cryogenic targets were operated in the August cycle and two target flasks were destructively tested. A small cryogenics laboratory is being prepared for full target testing. New controllers are being developed. The Saclay polarized target assembly with magnet was received and is being reworked.

A PDP-11/70 for general experimental data analysis arrived, as did a PDP-11/34 for the test channel. Program $Q$, the general-purpose dataacquisition code, was integrated into the newest RSX operating system. Upgrading of the MBD (computer-to-CAMAC) interface units began. Some 30 modules and other equipment from supplementary funds were purchased for the experimenters' equipment pool. A microprocessor-based A : iliary Crate Controller is under study, which would provide some programmable control or processing capability to a CAMAC crate. A report was issued from the summer study on data analysis at LAMPF.

\section{Research}

Nearly 600 data tapes have been taken on the reaction $\mu \rightarrow \mathrm{e} \gamma$ since last July. The experimental data so far look as if the stated goal of the experiment may be achieved if data-taking proceeds for 
another month, namely, an upper limit of a jew parts in $10^{10}$.

The data have now been analyzed for the elastic scattering of $800-\mathrm{MeV}$ protons on targets of $\mathrm{H},{ }^{12.13} \mathrm{C}$. ${ }^{38.60,62.64} \mathrm{Ni}$, and ${ }^{208} \mathrm{~Pb}$. Some inelastic data, including the $\left(4.44,2^{+}\right),\left(7.67,0^{+}\right),\left(9.64,3^{-}\right)$, and $\left(14.1,4^{+}\right)$ levels in ${ }^{12} \mathrm{C}$, the $\left(3.09,{ }^{1 / 2^{-}}\right),\left(3.68,{ }^{3 / 2} 2^{-}\right),\left(6.68,{ }^{5} 2^{-}\right)$, and $\left(7.55,{ }^{2} \cdot{ }^{-1}\right)$ levels in ${ }^{13} \mathrm{C}$, and more than 10 levels in ${ }^{53} \mathrm{Ni}$, have also been analyzed. The resolution obtained for the latter data was $<200 \mathrm{keV}$ full-width at tenth-maximum (FWTM), so that most of the levels were fully resolved. The elastic scaitering shows considerable sensitivity to specific details of the neutron and proton densities. The inelastic data show evidence for the spin-flip quadrupole mode of excitation of the nucleus.

Experiments have been ione on the influence of the nuclear environment on the cross sections for $\left(\pi^{ \pm}, \pi^{ \pm} n\right)$ and $\left(\pi^{ \pm}, \pi^{ \pm} p\right)$ reactions in the vicinity of the $\Delta(3,3)$ resonance. Results have been obtained for the reactions ${ }^{197} \mathrm{Au}\left(\pi^{ \pm}, \pi^{ \pm} n\right)^{196} \mathrm{Au}$ and ${ }^{25} \mathrm{Mg}\left(\pi^{ \pm}, \pi^{ \pm} \mathrm{p}\right){ }^{24} \mathrm{Na}$ over the energy range 100 . $300 \mathrm{MeV}$. The nuclear reaction cross sections show mass dependence. Although the ${ }^{25} \mathrm{Mg}\left(\pi^{ \pm}, \pi^{ \pm} p\right){ }^{24} \mathrm{Na}$ data are essentially identical to the analogous ${ }^{12} \mathrm{C}\left(\pi^{-}, \pi^{-} n\right){ }^{11} \mathrm{C}$ data, less dependence on energy is seen in the ${ }^{25} \mathrm{Mg}\left(\pi^{-}, \pi^{-} \mathrm{p}\right)^{24} \mathrm{Na}$ data compared with the ${ }^{12} \mathrm{C}\left(\pi^{+}, \pi^{*} n\right)^{11} \mathrm{C}$ data. In the case of ${ }^{197} \mathrm{Au}$, the cross sections are considerably larger and show even less energy dependence in the resonance region.

The ratios of the cross sections for the $\pi^{-}$- and $\pi^{-}-$ induced reactions in ${ }^{25} \mathrm{Mg}$ and ${ }^{197} \mathrm{Au}$ are compared with the analogous reactions in ${ }^{12} \mathrm{C}$ and for the free pion-nucleon interactions. A satisfactory theoretical interpretation of these data has not yet been given.

Additional studies are under way for the reactions on ${ }^{58} \mathrm{Ni}$ and ${ }^{142} \mathrm{Ce}$, in which both the $\left(\pi^{ \pm}, \pi^{ \pm} n\right)$ and $\left(\pi^{ \pm}, \pi^{ \pm} p\right)$ reactions are examined in the same nucleus.

An experiment on quasi-elastic scattering of 800 $\mathrm{MeV}$ protons was carried out at HRS. The experiment was a survey of the $\left(p, p^{\prime}\right)$ reaction (single arm) at $800 \mathrm{MeV}$ over a large range in target nucleus excitation, a large range in $\mathrm{A}\left({ }^{6} \mathrm{Li},{ }^{7} \mathrm{Li},{ }^{12} \mathrm{C},{ }^{27} \mathrm{Al},{ }^{40} \mathrm{Ca}\right.$, ${ }^{51} \mathrm{~V},{ }^{90} \mathrm{Zr}$, and ${ }^{208} \mathrm{~Pb}$ ), and a large range in scattering angle $\left(5^{\circ}-30^{\circ}\right)$. Targets of $\mathrm{CH}_{2}$ and $\mathrm{CD}_{2}$ were also run at each angle to provide normalization data.

The main areas of interest are: 1) quasi-elastic p$p$ scattering which is the dominant cross section, 2) quasi-elastic scattering of clusters (deuterons and alphas), and 3) discrete states of high excitation including giant resonances. A preliminary analysis of the data shows prominent bumps in the ${ }^{6} \mathrm{Li}$ and ${ }^{9} \mathrm{Be}$ energy spectra near the energy corresponding to free $(p, \alpha)$ scattering in the angular reşion from 25 to 30 degrees.

\section{Nuclear Chemisiry}

The floating-point processor for the Nuclear Chemistry Laboratory PDP-11/34 has been received and installed. Some preliminary programming was completed and an extensive checkout performed on the main interface to the Dual-Parameter Data-Acquisition System.

A gravity-operated, carrier-sensing electrical switch has been designed, fabricated, and is ready for lesting at the end of the "snake" at the Rabbit System Energetic Neutron Station at the A-6 beam stop. In the system Control Console, two timers for timing target exposures and six 3-step express transactions were wired in. Redesign of the Proton Station on Line $B$ has been completed and new components, including bellows, for the modification are being assembled. Four new heavy-duty terminals have been fabricated.

The major research activities involved target irradiations with $800-\mathrm{MeV}$ protons for three nuclear chemistry experiments, and numerous irradiations with beam stop neutrons for a fourth experiment.

In the area of theoretical support, the Third VEGAS Review meeting was held on August 26. 1977. A number of VEGAS/ISOBAR calculations relevant to several LAMPF pion experiments have been carried out. Consistently larger discrepancies are noted between calculated and measured results for $\pi^{-}$interaction cross sections than for $\pi^{*}$ cross sections. However, for another experiment, the backward peaking of $\pi^{0}$ production is reproduced by the calculation. The possibility that deformation of the ${ }^{12} \mathrm{C}$ nucleus may account for the $20 \%$ lower calculated total pion reaction cross section than observed at resonance is being investigated.

\section{Practical Applications}

At this stage the pion biomedical program has considerable momentum. Some 30 patients with 68 
tumors have each received 15-30 fractionated treatments. A computer-controlled treatment couch for dyrumic beam scanning has been installed. A catalog of therapeutically useful beam tunes is available with considerable documentation in terms of phase space, three-dimensional dosimetry, and linear-energy-transfer spectra. Dose and biological effectiveness of neutrons outside the treatment voiume were experimentally determined. and in vivo patient dosimetry is being developed. The treatment-planning code has continued to increase in sophistication and now uses computerized tomography (CT) x-ray data, acquired for each patient, to calculate bolus and dose distribution for the tumor volume.

In other practical applications experiments, experimental and theoretical work has continued on muonic $x$-ray analysis studies with emphasis on chemical effects and their applications. Improved experimental apparatus is being developed for the muon spin-rotation experimental program. The first phase of the proton radiography experiment was completed. The three-dimensional density distribution of a special phantom was reconstructed using both protons and a commercial CT scanner, with the result that for similar density distribution, the proton C'T scan required about four times less dose than the $x$-ray scan.

\section{Linac Technology}

The PIGMI $7-\mathrm{MeV}$ prototype accelerat or design is sufficiently advanced to permit some subsystem assemblies to proceed. The control system architecture is designed and component hardware is on hand and partially installed. The NOVA 3 central computer is operating from a half-finished control console. The equipment interface chassis, under development, will consist of assemblies of iive modules: binary command, binary data, analog command (digital to analog), analog data (analog to digital), and stepping motor command-andreadout.

The PIGMI permanent-magnet quadrupole (PMQ) design study has produced a fairly complete design and identified a suitable permanent-magnet material.

A layout of the PIGMI prototype installation at ETL has been prepared and the $250-\mathrm{kV}$ high-voltage set for the injector has been installed. The injector vicuum manifold is being fabricated and the ion source refurbished. A new ion beam design code confirms the PIGMI design.

An alternating-phase-focusing test cavity is ready for field studies. All components for PIGLET, the 45-MeV drift -t ube field-test ravity, are on hand and assembly has begun.

Some results are presented from studies of a model of the Gyrocon, a new microwave amplifier device. The calculations predict dc-to-rf conversion efficiencies $>80 \%$ are possible.

Another amplifier concept surfaced in contract studies performed outside as part of the Electronuclear Fuel-Producing Accelerator (ENFPA) evaluation. A gridded tube design with efficiency similar to the Gyrocon was proposed by one manufacturer. Since both capital cost and energy return are dominated by rf power generation cost and efficiency, the ENFPA study concentrated on the rf system. The studies have given formulas for capital cost per watt for rf power generation by tubes and klystrons as a function power and frequency.

\section{Management}

As a result of the planned shutdown in September, management decisions, and excellent cooperation by both LASL and outside user personneì, there was only a small overrun of operating costs for FY 1977. However, operating costs projected for FY 1978 indicate difficulties in meeting our commitments because of budgetary limitations.

During the two-month shutdown this quarter, major repair work in high-radiation areas was accomplished with no overexposures of personnel.

Close control of the movement of radioactive materials at LAMPF continued as a major effert. The crystal-detector portal monitors placed at 11 critical points throughout the site have picked up radioactivity on personneI. The Radiological Safe $y$ Comınittee met to review the control program, and recommended that additional units be procured for other locations.

The disposal of cooling water from the target cells and beam stops has long been recognized as a problem. which must be solved because of the accumulation of tritium. Through a series of meetings 
among LAMPF, Health Division, and Engineering Department personnel, a practical solution was found, and installation work started this quarter.

In parallel with the major staff efforts to complete repairs and modifications to the accelerator and experimental areas during the shutdown, significant progress was made in clearing up numerous minor electrical deficiencies and OSHA-type industrial safety items. Srfety inspections of all areas at LAMPF were conducted.

The annual election of officers for the Board of Directors of the LAMPF Users Group, Inc.. (LUGI) will be held at the Eleventh LAMPF Users Meeting on November 13-15, 1977. The positions to be filled are those being vacated by Barry $M$. Preedom of the University of South Carolina and Paul W. Todd of Pennsylvania State University, who are completing two-year terms of office. The Board will meet at LASL on November 15, and a meeting of the LLGI Technical Advisory Panel (TAP) will be held on November 16.

There are more than 1000 scientists, represent ing 311 institutions, who have joined the LAMPF Users Group. of whom 805 are from institutions other than LASL. Membership by type of institute represent ed is:

$\begin{array}{lr}\text { LASL } & 197 \\ \text { National or Government labs } & 121 \\ \text { U. S. Universities } & 391 \\ \text { Industry } & 57 \\ \text { Foreign } & 156 \\ \text { Hospitals } & 80\end{array}$

This Progress Report is in a new format, with activities taken from the group reports and assembled under major area headings, which should produce a better reference document.

\section{Publications}

J. J. Reidy, R. L. Hutson, and K. Springer, "The Use of Mesons for Tissue Chemical Analysis, "IEEE Trans. Nucl. Sri. NS-22, 1780-83 (1975).

R. L. Hutson. J. J. Reidy, K. Springer, H. Daniel, and H. Knowles, "Tissue Chemical Analysis with Muonic X Rays," Radiology 120, $193-98$ (1976).
A. (i. Law. "Linac Beam Core Modeling from WireScanner Data," Los Alamos Scientific Laboratory report LA-6894-MS (August 1977).

W. R. Thomas, "Charged Pion Production from Neutron-Proton Collisions at $790 \mathrm{MeV}$." Los Alamos Scientific Laboratory report LA-6962-T (September $197 \pi$.

M. D. Cooper, M. B. Johnson, and G. B. West. "Coulomb-Nuclear Interference with Pions," Nucl. Phys. A292, 350 (197\%).

\section{Papers Prepared for Publication; Papers Sub- mitted at Conferences}

J. J. Reidy, R. L. Hutson, H. Daniel, and K. Springer, "Use of Muonic $\mathrm{X}$ Rays for Nondestructive Analysis of Bulk Samples for Low- $Z$ Constituents." to be published in Anal. Chem.

L. Ray, G. W. Hoffmann. G. S. Blanpied, W. R. Coker, and R. P. Liljestrand, "Analysis of $0.8-\mathrm{GeV}$ Polarized Proton Elastic Scattering from ${ }^{208} \mathrm{~Pb},{ }^{91} \mathrm{Zr}$. and ${ }^{58} \mathrm{Ni}, "$ submitted to Phys. Rev. Lett.

L. Ray, G. W. Hoffmann, G. S. Blanpied, W. R. Coker. and R. P. Liljestrand, "Analyzing Power in Proton-Nucleus Elastic Scattering at $0.8 \mathrm{GeV}$." to be published in Phys. Rev. Lett.

S. Iversen, A. Obst, K. K. Seth, H. A. Thiessen. C. L. Morris, N. Tanaka, E. Smith. J. F. Amann, R. Boudrie. G. Burleson, M. Devereux, W. Swenson, P. Varghese, K. Boyer, W. J. Braithwaite, W. Cottingeme, and C. Fred Moore, "Neutron' Deformation Parameter by Comparative Study of $\pi^{*}$ and $\pi$ Inelastic," to be published in Phys. Rer. Lett.

G. W. Hoffmann. "Analysis of Elastic Scattering of $0.8-\mathrm{GeV}$ Polarized Protors from ${ }^{116 \mathrm{Sn}}$ and ${ }^{124 \mathrm{Sn}}$." submitted for publication in Phys. Lett. B. 
P.A.M. Gram, J. C. Pratt, M. A. Yates-Williams, H. C. Bryant, J. Donahue, H. Sharifian, and H. Tootonchi, "A Colliding Beams Experiment at LAMPF: Photodetachment Resonances in the $\mathrm{H}^{\text {- }}$ Ion," to be presented at M.I.T., Laboratory of Nuclear Science, Cambridge, Massachusetts, November 15, 1977.

D. E. Nagle, "Upper Limit for a Small ParityViolating Asymmetry in P.P Scattering at $15 \mathrm{MeV}$," presented at Ben Lee Memorial Int. Conf., Batavia, Illinois, October 1977.

R. E. Mischke, "Parity Violation in P-P Scattering," presented at the Seventh Hawaii Topical Conf. in Particle Physics, Univ. of Hawaii, Honolulu, Hawaii, August 1977.

D. J. Malbrough, C. W. Darden, R. D. Edge, T. Marks, B. M. Preedom, F. E. Berirand, T. P. Cleary, E. E. Gross, C. A. Ludemann, K. Gotow, R. L. Burman, M. A. Moinester, and R. P. Redwine, "Elastic Scattering of Positive Pions on ${ }^{16} \mathrm{O}$ at 40.0 and $49.7 \mathrm{MeV}$," to be published in Phys. Rev. C.

B. M. Preedom, C. W. Darden, R. D. Edge, T. Marks, M. J. Saitmarsh, K. Gabathuler, E. E. Gross, C. A. Ludemann, P. Y. Bertin, M. Blecher, K. Gotow, J. Alster, R. L. Burman, J.-P. Perroud, and R. P. Redwine, "The $\pi^{+}+d \rightarrow p+p$ Reaction at 40,50 , and $60 \mathrm{MeV}$," submitted to Phys. Rev. C.

M. P. Baker, R. L. Burman, M. D. Cooper, R. H. Heffner, D. M. Lee, R. P. Redwine, J. E. Spencer, M. A. Yates-Williams, D. J. Malbrough, T. Marks, B. M. Preedom, R. J. Holt, and B. Zeidman, "Mass Dependence of Pion Double-Charge Exchange," to be published in the proceedings of the meetings of the Am. Phys. Soc., Washington, LC, April 24-27, 1978.

H. A. Thiessen, J. F. Amann, C. L. Morris, N. T'anaka, L. W. Swenson, P. Varghese, G. Burleson, M. Devereux, R. Boudrie, S. Iverson, A. W. Obst, K. K. Seth, K. Boyer, W. J. Braithwaite, W. B. Cottingame, and C. Fred Moore, "Pion Scattering on ${ }^{12} \mathrm{C}$ at $162 \mathrm{MeV}, "$ ibid.

K. K. Seth, "Differential Study of $\pi^{+}$and $\pi^{-}$inelastic Scattering from ${ }^{18} \mathrm{O}, "$ ibid.
G. W. Hoftn. ann, "Exploration of Neutron Densities in Nuclei with 0.8-GeV Polarized Protons," invited paper, ibid.

M. E. Schillaci, "Negative Muon Capture in Binary Systems," to be presented at the 1977 Fall Meetings of the Amer. Phys. Soc., Miami Beach, Florida, November 21-23, 1977.

R. A. Jameson, R. S. Mills, and R. L. Cady, "Performance of Pulsed $805-\mathrm{MHz}, 1.25-\mathrm{MW}$ Klystrons into Mismatched Loads," submitted for publication in IEEE Trans. Electron Devices.

W. P. Lysenko, "Equilibrium Phase-Space Distributions and Space-Charge Limits in Linacs," to be presented at the Heavy Ion Fusion Workshop, Brookhaven National Lab., October 17-21, 1977.

K. M. Hanson and D. P. Boyd, "The Characteristics of CT Reconstruction Noise and Their Effect on Detectability," presented at the 1977 Nucl. Sci. Symp., San Francisco, California, October 19-21, 1977.

C. L. Morris, H. A. Thiessen, and G. W. Hoffmann, "Position-Sensitive Gas Proportional Chambers," to be published in Proc. of 1977 Nucl. Sci. Symp., San Francisco, California, October 1977; submitted to IEEE Trans. Nucl. Sci.

K. M. Hanson, J. N. Bradbury, T. M. Cannon, R. L. Hutson, D. B. Laubacher, R. Macek, M. A. Paciotti, and C. A. Taylor, "The Application of Protons to Computed Tomography," to be published in IEEE Trans. Nucl. Sci. and Proc. of the Nucl. Sci. Symp., San Francisco, California, October 1977.

M. Zaider, J. F. Dicello, and R. D. Hiebert, "A Pulsed System for Obtaining Microdosimetric Data with High-Intensity Beams," ibid.

M. Zaider and J. F. Dicello, "Investigation of the Microdosimetric Characteristics of Broad, Therapeutic Beams of Negative Pions at LAMPF," to be submitted to the 6th Symp. on Microdosimetry, Brussels, Belgium, May 1978. 
M. Zaider, J. F. Dicello, H. Amols, and S. Liıckstead, "An Analytical and Experimental Evaluation of the Contribution of $\delta$ Rays to the Absorbed Dose from 74-MeV Negative Pions," $i b i d$.

M. Leon, "The Energy Spectrum of Stopping Negative Mesons and the Concentration Dependence of Capture Fractions," to be submitted to Phys. Rev.

M. Leon and R. Seki, "Dynamic Electron Screening in Nuclear and Mesoric Atom E.1 Transitions," to be submitted to Nucl. Phys. Mesomolecular Processes in Matter, Dubna, USSR, June 7-10, 1977, sponsored by the Joint Inst. for Nucl. Res.

M. A. Paciotti, J. N. Bradbury, R. L. Hutson, E. A. Knapp, O. M. Rivera, and D. Laubacher, "Tuning of the Biomedica: Channel at LAMPF," presented at the 1977 Particle Accelerator Conf., Chicago, Illinois, March 16-18, 1977.

J. A. Helland and R. S. Kittell, "A Computer System for Radiation 'Therapy with Negative Pions," presented at the 6th Int. Conf. on the Use of Computers in Radiation Therapy, Goettingen, Germany, September 18-23, 1977.

J. D. Bowman and R. H. Heffner, "A Novel Zero Time Detector for Heavy Ion Spectroscopy," submitted to Nucl. Instrum. Methods.

K. M. Hanson, "Detectability in the Presence of Computed Tomographic Reconstruction Noise," to be published in the Proc. of the SPIE/SPSE Conf. on Application of Optical Instrumentation in Medicine VI, Boston, Massachusetts, September 25-27, 1977.

C. J. Sternhagen, J. D. Doss, P. W. Day, W. S. Edwards, R. C. Doberneck, F. S. Herzon, T. D. Powell, and G. F. O'Brien, "Preliminary Results Using Localized Current Field Hyperthermia in Oral Cavity Carcinomas and Skin Metastases," submitted to Cancer.
J. F. Dicello, M. Zaider, H. I. Amols, and S. Luckstead, "Recent Data for Pion Dosimetry, submitted to the 19th Annual Meeting of the Amer. Assoc. of Physicists in Medicine, Cincinnati, Ohio, July 31 August 4, 1977.

J. F. Dicello, "Dosimetry of Negative Pi Mesons," presented at the XIV Int. Congress on Radiology, Rio de Janeiro, Brazil, October 23-29, 1977.

\section{LAMPF Experimental Program Reports and Publications}

(Exp. 2) T. R. Fisher, J. A. Becker, B. A. Watson. H. Marshak, G. R. Burleson, M. D. Cooper, D. C. Hagerman, I. Halpern,. M. J. Jakobson, R. H. Jeppeson, K. F. Johnson, L. D. Knutson, R. E. Marrs, H. O. Meyer. R. P. Redwine, "Pion Cross Section Measurements on Aligned ${ }^{165} \mathrm{Ho}$ in the $(3,3)$ Resonance Region," Phys. Rev. C16, 2367 (1977).

(Exp. 90) E. A. Wadlinger, "A Generalized Chisquare Kinematic Fitting Procedure Applicable to Multiwire Proportional Chambers," Los Alamos Scientific Laboratory report LA-6573-MS (November 1976).

(Exp. 111) R. F. Petry, D. G. Shirk, J. C. Hill, and K. H. Wang, "Decay of Neutron-Rich ${ }^{45} \mathrm{Ar}$ and ${ }^{46} \mathrm{Ar}$," submitted to Phys. R.v. C.

(Exp. 129) W. R. Thomas, "Charged Pion Production from Neutron-Proton Collisions at $790 \mathrm{MeV}$," Thesis, to be submitted to Phys. Rev.

(Exp. 130) G. R. Smith, R. L. Boudrie, J. J. Kraushaar, R. J. Peterson, R. A. Ristinen, C. L. Morris, J. E. Bolger, W. J. Braithwaite. C. F. Moore, and L. E. Smith, "Energy Dependence of $\pi^{*}$ Induced Two- and Four-Nucleon Removal," Phys. Lett. 72B, 176 (1977). 
(Exp. 234) F. Milder, M. Blecher, K. Gotow, D. Jenkins, P. Roberson, R. L. Burman, M. A. Moinester, R. P. Redwine, F. E. Bertrand, T. P. Cleary, E. E. Gross, C. A. Ludemann, C. W. Darden, R. E. Edge, D. Malbrough, T. Marks, and B. M. Preedom, "Inelastic Scattering of $\pi^{+}$Mesons from Nuclei at $50 \mathrm{MeV}$," Phys. Lett. 72B, 159 (1977).
(Exp. 266) W. B. Gauster, R. H. Heffner, C. Y. Hunng, R. L. Hutson, M. Leon, D. M. Parkin, M. E. Schillaci, W. Triftshäuser, and W. R. Wampler, "Measurement of the Depolarization Rate of Positive Muons in Copper and Aluminum, "submitted to Solid State Communications. 


\section{FACILITY OPERATION}

\section{General}

The accelerator was in operation during all of August and the last two weeks of October, with 21 days devoted to research and 24 days for facilitydevelopment studies. The machine was shut down for the remaining 47 days for budgetary reasons and to permit major repairs and modifications.

The $\mathrm{H}^{+}$beam intensity was increased from $225 \mu \mathrm{A}$ to $300 \mu \mathrm{A}$ for the last seven research shifts. Machirie operation was reliable and stable at the higher level. Beam spill increased proportionately more than the current, as expected, but no significant increase in activation of accelerator components was noted. Use of negative-ion research beams was divided between the unpolarized $\left(\mathrm{H}^{-}\right)$and polarized $\left(\mathrm{P}^{-}\right)$, with 6 days of $\mathrm{H}^{-}$and 15 days of $\mathrm{P}^{-}$ beam. Machine availability during research shifts averaged $80 \%$ for the $\mathrm{H}^{+}, 63 \%$ for the $\mathrm{H}^{-}$, and $70 \%$ for the $\mathrm{P}^{-}$.

\section{Experimental Program}

Research-quality proton beams to Area A totalled $91000 \mu \mathrm{A}$-h during the quarter, as measured at the A-1 target station. An additional $57 \mu \mathrm{A}$-h of $\mathrm{H}^{-}$was delivered to Area $\mathrm{B}$. The cumulative total of proton beams delivered to the experimenial areas since dual-beam operation began in April 1976 is $731000 \mu \mathrm{A}-\mathrm{h}$. The $\mathrm{H}^{+}$beam intensity has increased by a factor of 30 during that time. Twenty-seven experiments received beam during the quarter, as shown in Table II-I.

\section{Operating Experience and Machine Downtime}

Machine operation continued to be reliable and stable at $225 \mu \mathrm{A}$. The relatively low availability $(63 \%)$ for the $\mathrm{H}^{-}$beam was due to overlap of the brief $\mathrm{H}^{-}$operating schedule with a major portion of the accelerator problems.

TABLE II-I

\section{LIST OF EXPERIMENTS THAT RECEIVED BEAM DURING THE PERIOD AUGUST 1 - OCTOBER 31, 1977}

\begin{tabular}{|c|c|c|c|c|c|c|c|}
\hline $\begin{array}{c}\text { Experiment } \\
\text { No. } \\
\end{array}$ & Channel & $\begin{array}{c}\text { Shifts } \\
(8 \mathrm{~h})\end{array}$ & $\boldsymbol{\mu A - h}$ & $\begin{array}{c}\text { Experiment } \\
\text { No. } \\
\end{array}$ & Channel & $\begin{array}{l}\text { Shifts } \\
(8 \mathrm{~h})\end{array}$ & $\boldsymbol{\mu A}-\mathbf{h}$ \\
\hline 14 & EPICS & 57.0 & 91000 & 267 & ISORAD & 48.5 & 54500 \\
\hline 27 & EPB & 33.0 & 1 & 270 & Biomed & 3.0 & 2540 \\
\hline 31 & Neutrino & 57.0 & 64000 & 271 & Biomed & 8.0 & 9450 \\
\hline 45 & ISORAD & 48.5 & 54500 & 273 & Biomed & 3.0 & 4100 \\
\hline 49 & HRS & 10.5 & 1 & 274 & Biomed & 17.5 & 20600 \\
\hline 82 & $\mathrm{P}^{*}$ & 28.5 & 40900 & 275 & Biomed & 21.5 & 26400 \\
\hline 86 & TTA & 57.0 & 91000 & 286 & $\mathrm{P}^{3}$ & 25.0 & 37700 \\
\hline 106 & AB-Nucchem & 1.0 & 9 & 299 & LEP & 8.5 & 13500 \\
\hline 111 & ISORAD & 4.5 & 5200 & 302 & PIP & 8.5 & 13400 \\
\hline 119 & $\mathrm{P}^{\mathbf{s}}$ & 2.0 & 2620 & 311 & HRS & 36.5 & $\ldots$ \\
\hline 144 & LEP & 48.5 & 78000 & 320 & $\mathrm{P}^{\mathrm{s}}$ & 2.5 & 2190 \\
\hline 206 & SMC & 5.5 & 6650 & 328 & SMC & 51.5 & 76500 \\
\hline 236 & Biomed & 1.0 & 1140 & 346 & HRS & 0.5 & -... \\
\hline 243 & AB-Nucchem & 3.0 & 22 & & & & \\
\hline
\end{tabular}


A summary of accelerator downtime during the 63 research shifts is given in Table II-II. The polarized ion injector, still in the development stage, had $63 \mathrm{~h}$ of downtime.

TABLE II-II

\section{QUARTERLY SUMMARY OF MACHINE DOWNTIME}

\begin{tabular}{l} 
Category \\
\hline 201-MHz amplifiers and transmission lines \\
805-MHz amplifier systems \\
Vacuum \\
Magnets and magnet power supplies \\
Interlocks \\
Injectors \\
Water \\
Computer control and data acquisition \\
Reference source \\
Beam stops, plugs, targets, strippers, scrapers \\
Beam diagnostics \\
Timing distribution systems \\
Miscellaneous (utilities, etc.)
\end{tabular}

TOTAL

\section{Downtime (h) \\ \% of Total}

\begin{tabular}{rr}
29.2 & 35 \\
14.7 & 17 \\
1.3 & 2 \\
7.2 & 8 \\
6.4 & 7 \\
3.2 & 4 \\
5.1 & 6 \\
0.7 & 1 \\
0.0 & 0 \\
7.2 & 8 \\
3.0 & 4 \\
2.2 & 3 \\
4.2 & 5 \\
\hline
\end{tabular}




\section{ACCEI.ERATOR}

\section{Development}

Accelerator development efforts focused on operation at higher beam intensities. Results in several areas were especially gratifying:

- The $\mathrm{H}^{+}$beam emittance was reduced by a third through optimization of ion source parameters. Improved matching techniques, in combination with the reduced emittance, have decreased the transverse phase space of the beam in the transition region (TR) to a degree that the TR no longer constitutes a limiting aperture.

- A low duty mode of accelerator operation was successfully employed for machine development experiments. The if amplifiers that drive the accelerator cavities were pulsed at the same repetition rate $(3.75 \mathrm{~Hz})$ as the beam, rather than the normal rf repetition rate of $120 \mathrm{~Hz}$, resulting in large savings in electrical power with no obvious invalidstion of experimental results.

-A demonstration run of an 8-mA, $625-\mu$ s pulse length, $800-\mathrm{MeV}$ beam at $3.75 \mathrm{~Hz}$ was successfully completed. 'The test was conducted in the low-duty rf mode. Beam transmission was greater than $99.5 \%$, and no low-momentum components were detected in the beam switchyard. There was no detectable beam spill in the TR. The measured emittance at $800 \mathrm{MeV}$ was $0.075 \pi \mathrm{cm}$-mrad in the horizontal plane and 0.052 in the vertical.

\section{Beam Dynamics Code Development}

Most of the CERN programs for calculating beam dynamics in the injector and the drift-tube linac have been successfully run at LAMPF. Additional effort will be required to adapt them for our needs.

The interactive dynamics program, TRACE, used on the control computer, has been modified to include the effects of a buncher on the transverse properties of the beam. This feature should allow better matching of the beam to the input of the linac.

The side-coupled linac simulation code SIMALAC is being revised and extended. The particle coordinates from the PARMILA code, which simulates the $201-\mathrm{MHz}$ linac and transport areas, may now be included in SIMALAC; the output graphics of SIMALAC were rewritten to use the Tektronix PLOT-10 software.

The second stage of code development provides for added capabilities. The effect of the earth's magnetic field on the transverse behavior of particle trajectories may be included. A new quadrupole subroutine has been installed that calculates dipole and higher multipole contributions to the magnetic field, and thus may simulate steering (dipole plus sextupole components) and quadrupole aberrations (various higher multipole components). Steering may be in either the horizontal or vertical plane at any quadrupole doublet element, and the design planes for any quadrupole doublet may be offset transversely or tilted to simulate misalignments. Trajectories and misalignments of the tanks and quadrupoles may be taken with respect to a smooth curve along the linac or with respect to the LAMPF (cartesian) coordinate system. Preliminary checkout of the coding for these features is complete, and an effort to compare calculated trajectories with measured behavior of the beam centroid in the 805 . $\mathrm{MHz}$ linac has been started.

Copies of the rf structures code SUPERFISH were provided to LLL and FNAL.

\section{Longitudinal Dynamics}

The results of $د t$ tuneups of the $805-\mathrm{MHz}$ linac during the last nine months have been used to conspare the effects at various peak currents. The settings produced by the tuneups using a $1-\mathrm{mA}_{\mathrm{P}}$ beam in June, July, and August were remarkably similar, in the sense that the module-to-module phases were within a few degrees throughout most of the linac. By examining the summaries of the $\Delta t$ results produced during these tunes, it is possible to estimate that the output energies were between 795 and $797 \mathrm{MeV}$. An attempt was made in June to tune using a 4-mA $A_{P}$ beam, and unsatisfactory results were obtained. A $\Delta t$ check on this tune using a 1 . $\mathrm{mA}_{\mathrm{P}}$ beam indicated that the output beam energy was $\sim 783 \mathrm{MeV}$. The tunes in March and April seem to have produced beam energies between 795 and $797 \mathrm{MeV}$, while 4-mAp tunes in January and February seem to have produced beam energies of $810 \mathrm{MeV}$ and higher. 
These results indicate that it is possible to produce good tunes that satisfactorily accelerate beams of higher intensities using a $1-\mathrm{mA}_{\mathrm{p}}$ beam, while tunes produced using beams of $4 \mathrm{~mA}_{\mathrm{p}}$ have not been satisfactory. The reason for this situation is unknown.

The $s t$ programs were modified to permit the $\Delta t$ procedure to be used in tuning the $201-\mathrm{MHz}$ linac tanks. The signals from the pickup loops in the 201 . $\mathrm{MHz}$ portion were satisfactory, and energy vs phase curves produced for Tanks 2,3 , and 4 all resembled the curves produced from computer simulations. However, when the actual $\Delta t$ measurements were made at Tank 3 , the phase and energy displacements (calculated from the measurements) were inconsistent with the energy vs phase curves. When Tank 3 was adjusted to give zero (calculated) energy and phase displacements, the resultant beam at module 5 had large displacements. Further investigations are planned.

\section{Horizontal Oscillation Control}

Successful control of the horizontal oscillation of the beam due to drift-tube vibration in the first two tanks was achieved. Using an algebraically decoupled controller, beam position was dynamically controlled during each beam pulse at position monitors located before and after the third tank. Correction steering modulation was applied to two sets of electrodes located in the $750-\mathrm{keV}$ transport line. The third tank was tuned to have a transfer function of an odd number of transverse oscillation quarter wavelengths by adjusting the quadrupoles. Considerable work remains to make the system operational.

\section{Transverse Dynamics in the 805-MHz Sector}

Two studies are under way which attempt to model certain aspects of transverse beam behavior. The first study is to determine the extent to which halos are created or broadened by mechanisms within the $805-\mathrm{MHz}$ sector of the linac. The initial approach to this problem is to examine the broadening of a Kapchinskii-Vladimirskii distribution from causes such as misalignments, quadrupole field aberrations, and sextupole components of steering fields.

The second study is to understand where particles lost from the acceleration bucket, longitudinally, will move transversely and finally hit the wall. The SIMALAC code was used to calculate particle trajectories. In the calculations done to date, the model used measured misalignments with respect to a smonth curve along the linac, and neglected the effects of quadrupole aberrations, steering (sextupole effects), and the earth's magnetic field; these will be added later. The calculations fall in three categories: low-energy losses, behavior at the edge of the bucket, and loss distributions along the linac. The low-energy study examined particles entering the linac below the design energy of $100 \mathrm{MeV}$. When the energy was at or below the bottom of the longitudinal acceptance, the particles lost tended to hit the wall around module 13. The edge of the longitudinal acceptance was found to be quite sharp, and the behavior right at the edge was very complex in the transverse planes. Because of the sharp edge, it is reasonable to assume that particles lost longitudinally get little net acceleration or deceleration, and therefore one may obtain representative transverse trajectories by using the model with the rf turned off past the energy of interest. Distributions of loss along the linac were thus obtained for particles within a specified initial emittance. This was done for various energies at which the particles stopped being accelerated. Some of the distributions for the horizontal plane are shown in Fig. III-1. The distributions generally show that lost particles with energies up to $\sim 190 \mathrm{MeV}$ hit the walls in the $805-\mathrm{MHz}$ linac; most particles with higher energies are transported on into the switchyard even though they are not accelerated.

\section{Beam-Profile Studies}

A new method was developed which uses the measured rms widths of the beam profile to obtain the transverse phase advance per module. The method also yields the initial orientation of the 805 $\mathrm{MHz}$ input emittance ellipse and the values of the $\alpha, \beta, \gamma$ parameters of this ellipse. A code now exists which gives best fits to the above parameters based on the assumptions that the input emittance can be described by an ellipse and that the matched ellipse 

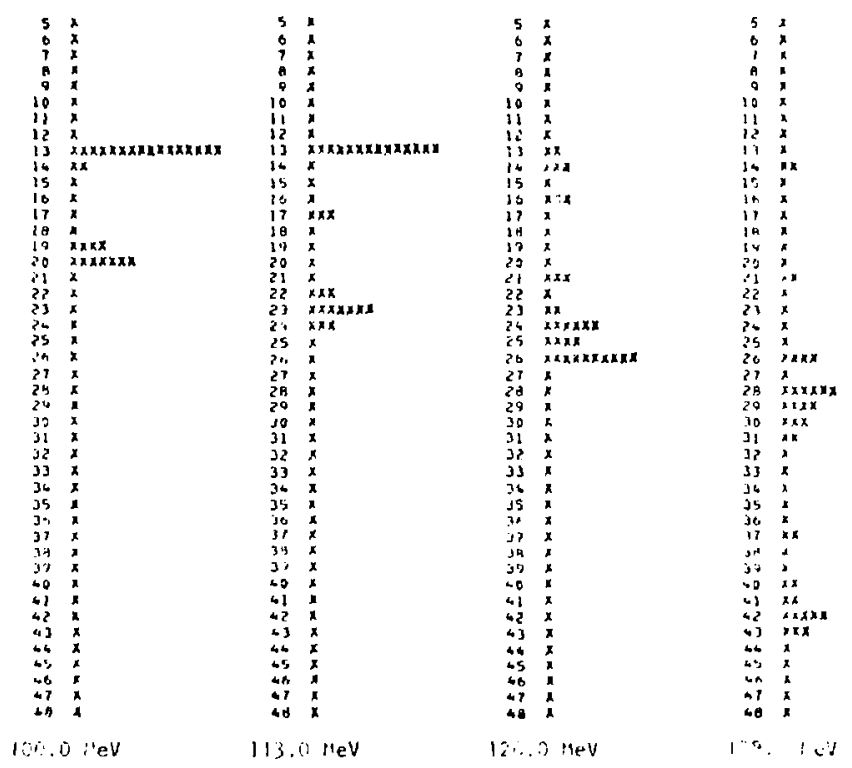

Fig. III-I.

Distribution of particle losses along the 805$\mathrm{MHz}$ linac as a function of energy at which acceleration is lost, calculated by SIMALAC.

of the linac is both known and constant over the region of measurements.

Using data taken during the July development period, we compared emittance parameters based on rms widths with those measured at EM-11. Both methods gave the same value of the initial orientation of the input ellipse, but gave differing values of the ratio of minor to major axis. Possible origins of this discrepancy are being explored.

Beam-steering experiments were repeated, and some puzzling aspects of our beam dynamics were observed. It appears that we usually have a beam. which is made up of at least two components. The amount of beam in the lesser component has been observed to vary between a few tenths of a percent to $-40 \%$ of the total beam. On one occasion, when profiles showed at least two beams were present with a production tune, we found that vertical steering at module 5 appeared to both change the relative orientation of the beams in emittance space and increase the observed separation of the profiles of the two components.

On three other occasions, beam profiles showed no evidence of two beams at wire-scanner locations $\mathbf{5 - 2}$ through 13-1 but showed evidence of a second small beam component when a large amount of vertical steuring was introduced at module 5 . These observations are consistent with the apparent increase in separation of the beam components caused by the application of vertical steering. An interesting observation is that in these cases where no second beam was apparent in the unsteered situation. we found that wire scanner 5-1, which is not homologous, did show evidence of a second small heam.

We hope that by including such things as aberrations and misalignments into the 805 simulator core. SIMALAC we will be able to reproduce these effects. The origin of these two beams is yet to be explained. However, increasing and decreasing the amplitude of the prebuncher does appear to increase and decrease the amount of secondary beam.

Earlier measurements showed unexplained fluctuations in the total beam current as measured by the sums of counts at the various wire-scanner locations. Tracking checks on the actuators were satisfactory. Checks of the constancy of surface conditions along each wire were made by steering the beam, but no variations were observed.

\section{Space-Charge Effects}

A report on equilibrium phase-space distributions has been completed. Some new results which help one to compare calculations with experimental observations include a calculation relating radial density profiles to space-charge potential expansion coefficients. Also, emittance values and other quantities corresponding to $90 \%$ of the beam have been extracted from the calculated distributions.

Analytical work in one degree of freedom has been started to determine the effect of an adiabatic variation of accelerator parameters during the process of acceleration.

The particle tracing code HOT was modified to include a dependence of the focusing forces on the independent variable. Tests show that accuracy is not sufficient for determining the effect of acceleration in the presence of space-charge forces. An areaweighting space-charge calculation will have to be incorporated in order to make this program yield useful results. 


\section{Injectors}

The injectors were on-line for production runs for only one month this quarter because of the scheduled shutdown. They were run for two weeks during the shutdown for development work. During the operating period, proton beams up to $300 \mu \mathrm{A}_{\mathrm{A}}$ were supplied simultaneously with either polarized or unpolarized $\mathrm{H}^{-}$beams; polarized beams up to $3 \mathrm{nA}_{\mathrm{A}}$ were supplied to HRS during the production period. During the shutdown, maintenance and development work were carried out on all three injectors.

Ground-loop problems in the data-acquisition system in the $\mathrm{H}^{+}$equipment dome were circumvented with fiberoptic isolation. Parity errors in chis system are now minimal.

New arcdown studies were initiated this guarter to try to determine mechanisms for high-voltage faults in the high-gradient accelerating tube.

Additional vacuum pumps were installed in the polarized ion injector in order to facilitate pumpdown in the presence of cesium contamination from the ion source. Work continued on interfacing the polarized injector control system to the main computer system. A permanent spin-reversing clock was installed and provides appropriate timing signals either in a spin-reversal or a rapid-quench mode.

The ion source test stand laboratory was refurbished, and construction of a new test stand is under way. This stand will permit operation of a variety of ion sources up to $100 \mathrm{kV}$, and will be used to develop brighter proton beams for LAMPF.

Computer codes, similating the extraction of ion bexms from a plasma and subsequent acceleration in an accelerating column, are now being readied for operetion at LASL. These codes have already been used for initial design studies.

\section{$\mathbf{H}^{+}$Injector}

The $\mathrm{H}^{+}$injector was run with a $650-\mu \mathrm{s}$ beam gate without difficulty, this quarter, and over $300 \mu \mathrm{A}_{\mathrm{A}}$ of $\mathrm{H}^{+}$beam was provided on target. The injector continues to be run at the $25-\mathrm{mA}_{\mathrm{p}}$ level for the tunes now being employed. Pulse widths up to $1000 \mu \mathrm{s}$ can be supplied with the present systems.

Intermittent parity errors in the $\mathrm{H}^{+}$injector data system were finally traced to ground loops between the arc-pulse modulator and the dome interface electronics. Extensive tests, carried out during the shutdown period, revealed that high-voltage interference from operating the dome at $750 \mathrm{kV}$ was not the primary cause of this problem, as originally presumed, but rather that ground loops were picking up interference from the arc-pulse modulator. The modulator was operated into a dummy load to preclude damage to the accelerating tube, and it was found that the parity error rate depended primarily upon modulator duty factor and was independent of dome high voltage. We tried various approaches to break up these ground loops in the dome, but without success. We then decided to completely bypass the interface system with a $10-\mathrm{m}$ fiberoptic cable, which was installed in the support legs for the equipment dome and connected cirrectly to the appropriate computer and $\mathrm{I} / \mathrm{O}$ lines. This modification has virtually eliminated the parity error rate.

A new gas-handling system has been installed that employs two palladium leaks in parallel. This system will permit higher gas flow to the $\mathrm{H}^{+}$ion . source and increase the time the leaks may be operated before bakeout is required. A new palladium leak system, with insulators of better quality, is being built, which is expected to eliminate the contamination problem with the palladium thimbles.

The wiring plant in the $\mathrm{H}^{+}$equipment dome was cleaned and many ground loops were removed. The drive lines from the arc-pulse modulator were replaced with larger lines and better terminations.

The drive motor on the MG set was replaced in order to repair an oil leak which resulted from uneven wear on one of the main oil seals. This problem was caused by a rough surface on tir main drive shaft and has been corrected. The original drive motor was replaced with a spare unit that had been obtained on excess from BNL. Some difficulty has been experienced with the spare motor because of bearing problems and because this motor draws considerably more current than the original motor. The wall breakers had to be replaced, as did the thermal overload elements. This motor will be replaced with the original motor for the next run cycle and will be used only as a spare.

Considerable preventive maintenance was performed on the Cockcroft-Walton and the associated high-voltage circuits. Several electrolytic capacitors 
now show signs of deterioration, and several have failed. New capacitors have been ordered. Some damage to surge resistors has also been found. The $\mathrm{H}^{*} \mathrm{C}-\mathrm{W}$ system has been operating for about nine years, and a detailed survey of critical components should now be carried out.

\section{Polarized Ion Injector}

An ongoing program to bring the operation of the polarized ion $\left(\mathrm{P}^{-}\right)$injector to its design current continued this quarter. Modifications and improvements brought the injector output up to $15 \mathrm{nA}_{\mathrm{A}}$ (one-half of the design goal for intensity) during the August production cycle. Only $\sim 20 \%$ of this beam was delivered to the experimental areas, compared to $\sim 70 \%$ optimum, because of tuning difficulties associated with instrumentation for low intensities.

The work on the $\mathrm{C}-\mathrm{W}$ high-voltage system was continued with installation of various control diagnostic readouts and interface circuits to the computer data system, together with work on documentation and spare-part inventories. The high-voltage interlock chain has been extended, and a series of diagnostic indicator lights installed to make troubleshuoting easier. The dome interlock light link has been moved to another leg of the C-W generator, where mechanical damage is less likely to occur. New pc cards have been laid out to replace the Haefely $\mathrm{C}-\mathrm{W}$ control cards with cards having standard LAMPF components and to provide several sets of spare boards.

An arcdown recovery circuit similar to that now in operation on the $\mathrm{H}^{+}$injector has bef $r_{1}$ installed on the $\mathrm{P}$ - injector. This system provides automatic resetting of the high voltage in the event of a fault.

Additional $\mathrm{C}-\mathrm{W}$ diagnostics and test points were installed, and a standard checkout procedure is being developed. Work still remains to be done to provide additional voltage ripple reduction and to carry out an absolute voltage calibration. In general, the high-voltage system now functions reliably and is fully operational.

A $350-\ell / \mathrm{s}$ blower pump was installed on the $\mathrm{P}^{-}$ duoplasmatron vacuum tank in order to facilitate pumpdown after opening the cesium cell to atmosphere. Initial operation indicates that the use of tilis blower facilitates turnon and reduces ion pump loading, but only long-term experience will deter- mine whether or not the ion pump lifetime will be extended by this addition.

Cooldown and warmup lines were added to the argon cryopumping system to reduce the beam downtime when the cryopump must be vented. Cooldown time should be reduced from five hours to three hours, and warmup time from six hours to one hour.

The argon cell turbopump failed catastrophically this quarter and was replaced with another turbopump of comparable speed.

A beam current toroid, identical in design to those used in the experimental areas, was installed on the polarized beam line. It is sensitive enough to "see" the unpolarized beam from the polarized ion source, but requires a suitable, low-noise preamplifier to be useful. Readout through the data system will soon be implemented, and will provide a convenient means of determining transmission through the accelerator for the beams from this ion source.

The spin-reversing clock has been replaced with a permanent unii. This system can be run either in a spin-reversal mode or in a fast-quench mode. It was used during Cycle 17 (August) to provide 3-min spin-reversal intervals and 2-s quench periods. These parameters can be adjusted to any desired values with thumbwheels installed on the clock. This design permits easy implementation of future modifications as new requirements occur.

A modification was made to the accel-decel electrode to increrse the production of the metastable beam. The decel electrode was mounted in a fixed position on the upstream end of the cesium cell, and the (R- $\theta-Z$ ) drive was used to move the accel lens in order to steer the $\mathrm{H}(2 \mathrm{~S})$ beam through the source more effectively. However, the proximity of the cesium cell to the high-voltage accel electrode resulted in considerabie arcing and high-voltage faults. An independent drive system has been fabricated for the decel electrode, and further modifications to optimize the operation of this portion of the ion source are now planned.

\section{Injector Development}

Several approaches have been initiated in the development of new injector systems, and work has continued in the study of properties and tunes of the ion beams now being run at LAMPF. 
A careful study of emittance damping with simultaneous $H$ and $H$ beams being run with the same linac tune was carried out this quarter. The $H$ hean was momentum analyzed, and a $100-\mu A_{1}$, beam having a single-ion species with the same phase-space area as a $5-\mathrm{mA}_{\mathrm{p}} \mathrm{H}$ beam was prepared. These two beams were then simultaneously matched and accelerated through the transition region, and emittance measurements were carried out for both beams in both transverse planes at the input and exit of the linac. A significant difference in the emittance damping was observed between the $\mathrm{H}^{-}$and $\mathrm{H}^{-}$beams. The $\mathrm{H}^{-}$beam exhibited little emittance growth. In fact, in the horizontal plane the emittance damping was essentially that predicted by the $\beta \gamma$ momentum factor. The brighter $\mathrm{H}^{*}$ beam exhibited significant emittance growth (growth factor of 2.5 in the horizontal plane and 3.5 in the vertical plane). For both beams there is still appreciable difference in the damping between the horizontal and vertical planes so that the dependence of emittance growth on beam brightness is complicated by transverse alignment and matching problems. These observations should be carried out on APF structures where the beam brightness can easily be varied with sieves and where circular symmetry will make observations of emittance growth unambiguous.

Arcdown studies were initiated on the $\mathrm{H}^{+}$injector in order to try to identify mechanisms causing highvolt age faults. A multichannel analyzer was set up and run in the multiscaling mode ' $x$ ith a dwell time of $10 \mu$ s per channel. Multiscaling was initiated by the beam gate and, with 512 channels available, a time period of $5.12 \mathrm{~ms}$ was observed after the start of each beam gate. The system was set up and run for several weeks during the production run. The data obtained are shown in Fig. III-2. The results were not anticipated in that the maximum fault rate occurs at $2013 \mu \mathrm{s}$ after initiation of beam gate rather than at the beginning and end of beam gate where beam spill in the column is a maximum. One possible mechanism being considered is electrode heating due to an errant pulse.

Work has continued this quarter on the effort to increase the brightness of the prcton beam obtained from the $\mathrm{H}^{+}$injector. The old ion source test stand used for $\mathrm{H}^{-}$source testing and the polarized ion source laboratory are being modified and refurbished in order to study ion beams that will be

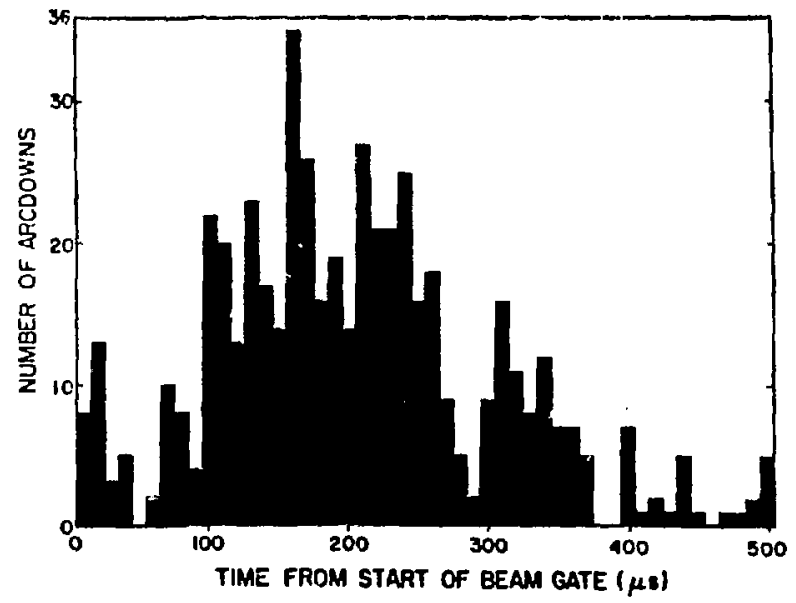

Fig. III-2.

Distribution of arcdowns during the beam pulse for the $H^{+}$injector.

extracted from several $\mathrm{H}^{+}$ion sources. The floor of the laboratory was covered to make it level for the construction of a high-voltage test stand. The test stiand ion pump and roughing system were rebuilt and the vacuum chambers were hydrogen cleaned. A four-gap glass and stainless steel high-voltage column has been constructed that will allow the ion source to be isolated at a potential of $100 \mathrm{kV}$. This design will also allow several acceleration gaps to be used in the column, so that the ion beams can be studied in a geometry that approximates the first few gaps in the $\mathrm{H}^{-}$injector. Suitable ion beam diagnostic equipment, located beyond the column in the grounded vacuum chamber, will be used to measure the ion beam emittance, density profile, and molecular composition. The emit tance and density scanning device to be constructed will be patterned after that developed by P. Allison ${ }^{1}$ in his surface ionization source program. Also, ? iangmuir probe will be set up to measure the ion current density profile in the plasma expansion cup with no accelerating voltage on the column.

In addition to this experimental program, several computer codes are being implemented at LAMPF to carry out calculations of ion beam trajectories in proposed ion sources and accelerating tubes. One of the first codes to be implemented is SNOW,? which is being developed by J. E. Boers at the Sandia Laboratory. This code simulates the extraction of ions rom the plasma in a plasma expansion cup in 
cylindrically symmetric geometries. In this calculation, ions are injected into the plasma at a specified injection plane in the expansion cup with a currentdensity distribution that can be specified, e.g.. by. the experimentally measured distribution. This code has already been used to check the design calculations on the convergent beam geometry for PIGMI.

Caiculations on the present $\mathrm{H}^{-}$injector with this code are planned for the next quarter, and these calculations, as well as the ion beam measurements to be made on the new ion source test apparatus when it is completed, will be used to make the changes necessary in the $\mathrm{H}^{+}$injector in order to increase the brightness of the proton beam.

\section{RF Systems}

\section{1-MHz}

An average of $785 \mathrm{~h}$ was accumulated on each of the $201-\mathrm{MHz}$ rf modules, $39 \%$ of the total time available during the quarter.

Two 4616 rf driver tubes failed during the quarter. One failed with a control grid-to-cathode short and the other lost vacuum while being run at the test stand. The tube that lost vacuum was returned to RCA under warranty for a failure evaluation and repair. $R C A$ reported that both it and the tube that lost vacuum the previous quarter had holes burned in the anodes. Both tubes were of an older construction that utilized copper anodes. RCA informed us that they will no longer rebuild 4616 s with copper anodes, although they will honor the warranty on these two tubes.

Two 4CW250 000 switch tubes were retired when they exhibited high voltage drop. One 4CW250 000 which was returned by the Navy after being loaned to them, was discovered to have a control grid short. The Navy has agreed to replace the tube with a new one.

There was a flashover in the crowbar resistor oilcooling tank in Capacitor Room 2, releasing̨ enough stored energy to generate a shock wave in the oil to severely deform one end of the tank. A container was designed and installed in Rooms 2, 3, and 4 during the fall shutdown that will catch the 300 gallons of oil in case the cooling tank ruptures during a fault. In addition, the highest voltage stress point in the tanks was relieved by removing the shields that encompassed the high-voltage divider.

During the disassembly of the $36-\mathrm{cm}$ transmission line in module 4 (in an attempt to find a breakdown point), we found that several of the Rexolite center conductor supports in the line shcwed evidence of heating. A design change has been initiated that will allow removal of these supports.

The Rexolite vacuum barrier on Tank 4 was also changed. Although it had accumulated $8000 \mathrm{~h}$ of running time, there was no severe damage. The Rexolite output windows on the 7835s in modules 3 and 4 showed damage, caused by high duty runs, and were replaced.

Some more cold probing was done on the dc blocker for the 4664 output cavity in an effort to find the cause of $\mathrm{rf}$ leakage from the cavity. The cold probe showed that the blocker length is correct, but it appeared that shorting the plate at the Delrin insulator point greatly improved the effectiveness of the blocker, as did increasing the capacitance at this point with lumped capacitors. As a result of these tests, high-voltage vacuum capacitors have been ordered and will be installed in an attempt to reduce the leakage.

The sampling loops along the side of Tank 2 were instrumented with crystal detectors and sampleand-holds. Analog recordings are being made under conditions of varying duty and tank behavior to evaluate apparent field changes noted during development runs.

Fabrication of parts for redesigned 201-MHz tuning slugs is nearing completion. Initial conversion of six tuning slugs is expected to provide reliable backup for operation of these units in the closedloop mode. One tuning slug per $201-\mathrm{MHz}$ tank is to be operated in this manner.

A run was made with the power, through the fast phase shifter, reduced by $10 \mathrm{~dB}$ in an effort to isolate phase noise problems. No improvements in the noise were noted at the reduced power, but instrumentation to measure phase noise would need to be developed to make quantitative measurements. The ports were reversed on the phase package to change the power level through the fast phase shifter for this test. A change in power to the amplitude control system with changes in phase position were noted while running in this configuration. Also, changes in closed-loop gain of the amplitude control 
loops were noted when the pulsing rate was not synchronous with the power line. This gain change was traced to the filament voltage on the first tube stage of the PA Modulator Driver.

The final version of the oil-immersed screen supply transformer for the IPA Modulator was completed and has logged 256 hwh at the test stand. Sufficient material is now on hand to start production on these transformers as well as the new set of transformers for the PA Modulator.

Two 4616s, two 4664s, and three 4CW250 000 tubes, as well as two IPA Modulators, were tested and processed at the test stand during the quarter. Operation of the stand was minimal during the fall shutdown.

\section{5-MHz}

The VA-862A klystrons have accumulated 1090500 filament hours (fh) with 16 failures. The average age of those in service is $19500 \mathrm{~h}$. An expected average life for these tubes is $\sim 28000 \mathrm{~h}$. Only two of the Varian tubes required processing at ETL during the quarter.

The L-5120 klystrons have accumulated $200500 \mathrm{fh}$, with 22 failures. The average age of those in service is $9500 \mathrm{~h}$. There are two klystrons in Sector $\mathrm{H}$ with $18000 \mathrm{~h}$ or more, yet the average life is $\sim 4500 \mathrm{~h}$. Five of the Litton tubes were processed at ETL during the quarter.

Repairs were started on four klystrons during the quarter, with one successful completion. One klystron is still being processed, and one failed tue to a punctured ceramic ring during hi-pot tests. The fourth repair was a VA-862A high-voltage section on an L-5120 body. The tube was tested and worked well with $51 \%$ efficiency, good gain and characteristic curves, and no glitches. It is being rebuilt again because of vacuum and water-leak problems. Five klystrons are in the repair cycle now, with two ready for the final repair stage in the bakeout oven. The remaining three will take longer with drift-tube or collector repairs required.

The LPT-44 switchtubes have accumulated $1310000 \mathrm{fh}$ with 41 final failures. The average life of those in service is $13000 \mathrm{~h}$ with a life expectancy of $\sim 14000 \mathrm{~h}$. The switchtubes are reprocessed five or six times during their life, and discarded when they can no longer be restored. The final processing step now is a test run in a modulator at ETL.

The klystron pulse amplifier and bias circuit modification to conserve power by turning the klystron pulse off earlier was completed in all sectors.

This quarter the number of klystron crowbars decreased $50 \%$ relative to last quarter to an average rate of 1.9 per 1000 hvh.

Failure of the 24-V-dc sector power supplies was traced to insufficient cooling, so the fan in the supply was replaced with a larger capacity unit. At the same time, the exposed ac-input connection was replaced with a connector meeting LAMPF standards.

The automatic rundown modification and the installation of sealed relays in the quad magnet power supplies have been completed. These modifications should eliminate most of the power-supply restart problems.

The tank resonance system modifications have been completed and the system has been calibrated. All tank temperature controllers are interchangeable to better than $0.06^{\circ} \mathrm{C}$.

In October all zero-power and full-power setpoints were adjusted for minimum reflected power. This was previously done last January. In comparing the January and October settings for zero-power, it was found that the average settings changed by $0.18^{\circ} \mathrm{C}$. Individual differences varied from zero to $0.56^{\circ} \mathrm{C}$. This would suggest that a more precise indication of reflected power than can be read on an oscilloscope is required.

The third set of data from the RG-218 cable insulation growth test continues to indicate that the insulation expansion is associated with immersion in warm oil. The tests will continue until the insulation expansion ceases.

The coaxial cables that run from the accelerator tank to the phase package have had a history of connectors being broken while being connected or disconnected. This breakage was due to the extreme stiffness of the heliax being used. The problem has been eliminated by replacing all cables with type FSJ1-50 superflexible heliax.

The Belden 8871-hv cable under test at the ETL klystron test stand has $13990 \mathrm{hvh}$. 


\section{Low-Level RF Systems}

Extensive upgrading of the $201-\mathrm{MHz}$ and $805-$ $\mathrm{MHz}$ source was carried out during the fall shutdown. All tube-type buffer amplifiers were replaced with redundant solid-state units. These new amplifiers are located on the multiplier c.lassis and are switched with the multiplier units.

Both oscillator/multiplier strings and their associated patch panel have been relocated in the center rack in the screen room. This has freed the left-hand rack for the future addition of a redundant phase and amplitude system. In addition, all coaxia! cables in the screen room were rerouted and the existing cable was replaced with type FSJ1-50 superflexible heliax. These changes appear to have reduced the noise on both systems.

The source power amplifier control system is being revised so that each amplifier string will have a central control panel. When this revision is completed, a string can be turned on or off and the interlocks summed at one location.

All 201-MHz solid-state IFAs have been received. The units are now installed and operating in the bunchers and in modules 1-4.

Based on performance in Sector D during the last quarter, the decision was made to proceed with the construction of a combined $805-\mathrm{MHz}$ solid-state IFA and strip-line fast phase shifter in a common chassis.

\section{Phase and Amplitude Control Systems}

A new rf-permissive system which will allow blanking of selective periods during the rf gate was completed. After careful evaluation of a prototype in Sector $H$, the unit was fabricated and is now operational in all sectors.

The phase subsystem modification and refurbishment program is now complete throughout the accelerator. Only the spare units remain to be modified.

All 201- $\mathrm{MHz}$ fast phase shifters have been modified to provide more linear loss curves. This was done by reducing the phase-shifter amplifier power-supply voltage by $25 \%$. All units have been recalibrated under the new operating conditions.

\section{Components}

The injector $\mathrm{H}$ line prebuncher was aligned to beam-line axis using two bore targets. Subsequent beam-line components can now be installed and their alignment referenced to the buncher cavity.

Alignment checks were made on four beam boxes located between Tanks 3 and 4 of the $201-\mathrm{MHz}$ sector.

Tank lengths and intertank spacings were measured on 805-MHz modules 11 thro:ıgh 14 using the $100-\mathrm{m}$ tooling tape and jig transit

Outgas rates and pumping speed measurements were continued on the $201-\mathrm{MHz}$ tanks. These tests provide useful information on the cleanliness of the vacuum system and pump performance, and serve as a guide for scheduling maintenance and pump rebuild.

The TR-BM-02,-06 bending-magnet drawing revisions are complete. Parts required to assemble two spare coil assemblies are $\sim 75 \%$ complete.

A new fence for the TR has been designed and drawn. In addition to providing protection for personnel and equipment, the design permits access to any area by one person without removing the entire unit, and it can support a limited amount of sheet lead shielding.

Numerous water hoses were replaced in the TR as a result of routine inspections. A large portion of the hoses that were replaced have been in service since initial installation.

As a result of preliminary studies using the 8.5$\mathrm{kG} \cdot \mathrm{cm}$ WNR steering magnets that were temporarily installed in the main line, two nesw steering magnets specifically designed for this application were fabricated and installed during the October shutdown. The new units provide steering fields of $55 \mathrm{kG}$-cm (Fig. III-3).

Drawings are nearing completion for the new steering magnets to be installed in the side track area, immediately upstream and downstream of TR-BM-07.

Fabrication of the new $33-\mathrm{cm}$ beam boxes is near completion. The boxes and support structure for use in bott the accelerator and in PIGMI are being fabricated and should be completed by the middle of November. Installation in the accelerator between Tanks 2 and 3 is scheduled for the December shutdown. 


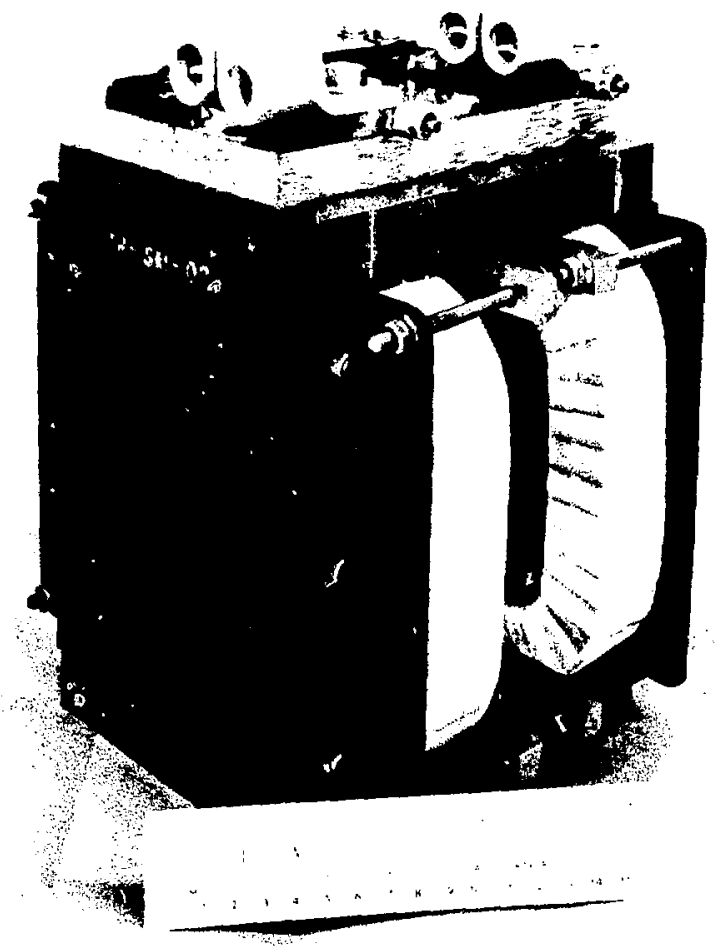

Fig. III-3.

Transition region steering magnet.

Six leaky $805-\mathrm{MHz}$ window spacer rejects were reworked to salvage three usable spacers. This was accomplished by cutting the spacers in half, discarding the leaky part, and welding the good sections together.

\section{Instrumentation and Controls}

\section{Beam-Position Monitors}

Construction of the bins, terminators, cables, and power-supply modules was initiated. Problems with drift and slew rate in the integrated circuit detector chip were resolved late in the quarter by circuit changes and choice of better grades (pretested) of components. There is still some concern about the percentage of good units that can be attained in a production run. Design of the external detector circuitry and the current normalization divider was completed. All parts wil he in the fabrication stage early next quarter.

\section{Wire Scanners}

Six prototype; of the multigain-amplifer package are installed in the beam tunnel and are operating. Additional cables have been installed in the 805$\mathrm{MHz}$ linac which will enable us to use these prototypes at modules 5 through 13 . In the $201-\mathrm{MHz}$ linac, cables have also been routed to the entrance of Tanks 1-4. A wire scanner and a multigainamplifier package has been installed and checked out at the beginning of Tank 4 .

A self-contained beam-pulse simulator, which supplies an input signal to the multigain-amplifier package, has been designed and built and has been most useful in troubleshooting. As a result of careful shielding and use of the power supplies in the particular package being tested, we find that this simulator has none of the noise and pickup problems seen in other generators.

We have incorporated a number of additions into the multigain-amplifier code which enables us to make profile measurements in the $201-\mathrm{MHz}$ linac, with its different set of computer channels, and to make use of the biasing and grounding capabilities of the multigain-amplifier package. The dual-gain feature of the $805-\mathrm{MHz}$ wire-scanner amplifier modules was upgraded to include computer control of the gain charge. A small pc card containing the control circuitry was installed in each amplifier module.

\section{Actuator Maintenance}

During the fall shutdown, 30 actuators in Sector $\mathrm{A}$ were removed from the beam line for major maintenance and adjustment.

\section{Emittance Gear}

It has been reported ${ }^{3}$ that a proton beam can be neutralized by elertrons when making emittance measurements because of electrons from beam striking the emittance jaws. Some of the electrons then 
backstream, and their presence distorts the original beam-intensity distribution. Typical observed effecis are emittance shapes which are both rotated and reduced in size. We have made emittance measurements at EM-2 wich various applied voliages on the EM-2 jaws. We observed a voltagedependent rotation of that portion of the beam which lies rear the borders of the emittance phase space. The main core of the beam appears to be unaffected. Since the beam conditions were somewhat unstable during this experiment, the experiment was repeated; these data are being analyzed.

Ceramic harp boards with carbon wires were fabricated and installed on all harp actuators except harp \#4, where a ceramic board with silicon-carbide wires was fabricated and installed. This new type of wire presented some rather severe fabrication problems. Additional experimentation in soldering, plating, and winding of silicon-carbide wire must be done if operational tests of harp \#4 indicate that a change to this wire is desirable. A wire-scanner actuator using silicon-carbide wires was also installed in module 9 for evaluation.

The design and fabrication of a motor-driven, computer-controlled rotary harn switch is $80 \%$ complete. This switch will automatically disconnect all harp signal wires except the one that is being driven into the beam.

To scan both $\mathrm{H}^{+}$and $\mathrm{H}^{-}$beams at the end of Tank 4, harp \#3 and the double-fixed-slit EM-7 jaw must be mounted on 13-cm stroke actuators. Additional actuators have been ordered to accomplish this.

\section{$\Delta$ t System}

The $\Delta t$ matrix switch encoder was redesigned and one new pc card is being drawn in preparation for production. The automatic $\Delta t$ simulation system hardware was installed in the $\Delta t$ system. New $\Delta t$ phase controllers were installed and checked out. These controllers have an adjustment for beam gate delay designed onto the card.

\section{Beam Scrapers}

Two new beam-scraper designs utilizing watercooled graphite jaws have been completed. The units have been installed at the $40-$ and $70-\mathrm{MeV}$ scraper stations for evaluation.

\section{Fast-Protect System}

A major modification of the fast-protect and rfpermissive interlock systems was implemented. The change permits blanking of troublesome turn-on and turn-off transients present on phase and amplitude control systems when high-peak beam currents are accelerated.

Test points to provide for easier maintenance were also installed in all fast-protect chassis. A new fast-binary command card was developed; handwired prototypes are in service and art work for a pc board is in progress.

All activation-protection units were modified to require higher interstage voltage on the photomultiplier tubes to provide more stable operation of the power supplies. Half of the activationprotection units were further modified to provide a linear response for radiation intensities well below the trip level, and all of the units were recalibrated against a ${ }^{80} \mathrm{Co}$ source.

\section{Interlocks}

Run-permit interlock systems for all three injectors were documented. The 201- $\mathrm{MHz}$ quad-magnettemperature interlock system was rebuilt. Personnel safety systems for caves in experimental Area A were modified to prevent gates from being left open during restricted-access entries and to require a longer duration for the warning horn.

The run-permissive interlock system in beam areas B, C, and EPB were completely rebuilt. Training documentation and qualification sheets were generated for the experimental area water systems and secondary beam-line interlocks. 


\section{CCR and Computer}

\section{New Interface Unit}

A "smart" (microprocessor-controlled) data link (MCDL) is being developed to replace the data links connecting the control computer to four satellite computers. The first two MCDLs in a CAMAC double-width pc version were fabricated and tested. One link was installed between the control computer and the storage scope display system; the ot her was kept as a spare and used for further testing. Parts were ordered for four more units.

\section{Master Timer (MT)}

Since the accelerator shutdown provided one of the few opport unities to install a major piece of new equipment, the computer activites this quarter were concentrated on the master timer (MT) and the new disk system.

The normal technique for installing a new system is to phase it in with the old system carrying some reduced percentage of the load until the new system is fully commissioned. In the case of the master timer, this approach was not feasible because new operating modes exceedine the capabilities of the old MT were instituted concurrently with the integration of the new MT. Thus, the changeover had to be abrupt and complete.

In anticipation of the conversion, the operating requirements for the upcoming cycle were specified and the necessary software was developed for both the control computer and the microprocessor in the MT. The read-and-set executive program calls in the SEL-840 were rewritten. Programs were written to display the operating parameters of the MT and to provide options for controlling them. Communications software between the SEL- 840 and the MT were implemented. The control program in the microprocessor of the MT was "burned" in a PROM and installed. Since the MT is a key element in the acquisition of data from the accelerator, extensive modifications had to be made in the RIU driver to accommodate the new MT. A program to display the timing relationships among the nine MT gates was developed.

All of these developments were validated as thoroughly as possible without the accelerator in operation. At the start of the turnon, a class of problems was discovered, all related to the fact that during development the rf gates no longer occur necessarily at $120 \mathrm{~Hz}$. This problem was solved by modifying the software to remove the axis-crossing restrictions. Other problems associated with $\mathrm{H}^{+}$and $\mathrm{H}$ beam gates not necessarily falling on the same cycle were resolved by setting the MT so that the $\mathrm{H}$ and $\mathrm{P}^{-}$beam gates follow the $\mathrm{H}^{+}$beam gate. Major changes in the software supporting the MT can be expected for some months to come.

Preliminary work began on a second (backup) master-timing unit. The construction of this unit is proceeding slowly so that improvements, gained from operating experience with the first unit, can be incorporated in the second.

Five people attended a series of training lectures dealing with the master timer hardware. Documentation for the system is being kept up-to-date. Training sessions on the software will begin next quarter.

\section{New Disk System}

The original disk system on the control computer is being replaced by a new system to increase the data-storage capacity from 3- to 40-million 24-bit words. The new system consists of a disk controller with three disk drives, all interfaced to the control computer through a PDP-11/34 which supports the fille-handling software under RSX-11M.

The new disk system was put on-line to the control computer. For the next production cycle, the new system will handle a group of noncritical fł̣les. This approach will permit a shakedown of the system under realistic conditions without jeopardizing operations.

The integration of the new disk system required the solution of two problems. The first had to do with transferring large blocks of data from the control computer. This problem was solved by modifying the SEL-840 to PDP-11/34 interface so that the PDP-11 could fragment a long block from the control computer into several smaller blocks and store them away. The second problem lay in Digital's RSX-11M software. Version 3 of this package was released with so many errors that it took the better part of a month to get the system running. 


\section{Program Maintenance}

Numerous programs received attention to correct minor deficiencies or to update them to reflect recent hardware changes. Among them were the programs for the harps, the SY wire scanners, the rf monitor, the Run-Permit system, the water system, the beam strippers, and the beam history summaries.

Advantage was taken of the six-week shutdown to troubleshoot a long-standing problem in the control computer, namely, lost interrupts in the block transfer channel (BTC). The lost interrupts associated with BTC4, which services the RIU, were traced to two separate causes, both of which were corrected by changes in the supporting software. The other BTCs are connected to rotating peripheral devices, i.e., a disk, a drum, and a magnetic-tape system. Tests demonstrated that when transfers are in progress on both the disk and the drum and programmed $I / O$ instructions are executed. then one of the interripts may be lost because a word from the rotating storage was missed. This contention for the $\mathrm{I} / \mathrm{O}$ bus is symptomatic of the heavy load on the control computer. The conversion to the new disk system will eliminate the problem because the interfacing scheme has more buffer storage.
Work began this quarter on a new series of programs to save, restore, list, compare, and plot accelerator operating parameters.

\section{Third Console}

In connection with the new operator's console, a set of programs was written to serve as a driver and provide various test and exercise functions for the new color-character-display scope. This system 'vill provide all of the capabilities of our earlier (and now discontinued) $\mathrm{CCI}$ scopes plus a few new features such as background characters and special characters.

\section{REFERENCES}

1. P. A. Allison, "Experiments with a DudnikovType $\mathrm{H}^{-}$Ion Source," Symp. of Negative Ion Beams, Brookhaven National Laboratory, Upton, New York, September 20-26, 1977.

2. J. E. Boers. Bull. Am. Phys. Soc. 22, 1081 (1977).

3. L. R. Evans and D. J. Warner, "A Critical Study of Emittance Measurements of Intense LowEnergy Proton Beams," Nucl. Instrum. Methods 104, $61-70$ (1972). 


\section{MAIN BEAM LINES}

\section{Development}

\section{Phase-Space Measurements in Line A}

The previous procedures for obtaining phasespace parameters involved special beam tunes and reduced beam current in Line A-Direct. A new procedure has been developed which extracts the necessary information from the standard production beam in Line A-South. The wire scanners can be run during production (at least up to $300-\mu \mathrm{A}$ average current). The measured emittances agree with measurements in Line A-Direct to within the statistical uncertainty. ${ }^{1}$ The correlation parameter differs from that obtained from measurements in Line A-Direct. This is probably because of lack of knowledge of the gradient and effective length of the quadrupoles which are included in the fitting procedure. The Line A-Direct procedure uses four profile measurements in a drift space. The new procedure uses profile measurements from six or seven wire scanners in a section of beamline which includes five quadrupoles and five bending magnets.

\section{A-5 Proton Beam Spot}

The radiatively cooled pyrolytic graphite target at A-5 has failed on two occasions in a way that suggests that the density of energy deposition may have been the cause. Therefore, we have doubled the horizontal spot size and increased the vertical size by $\sim 30 \%$. The nominal proton beam at $\mathrm{A}-5$ is now approximately circular and characterized by $\sigma$ $\sim 5 \mathrm{~mm}$.

\section{Instrumentation and Controls}

\section{Beam Current Monitors}

Most of the work on a prototype of a new beam current monitor (BCM) amplifier-digitizer was completed. Three BCM amplifiers, three BCM power supplies, and two expandable NIM bins were built and tested. If the trial installation next quarter is successful, a complete set of documentation ischematics, pc.hoard layouts, negatives, silkscreen masters, machiring and lugging drawings, etc.) has been prepared to facilitate the production of hardware for enough units to upgrade the existing system from the SY through A-6.

\section{Harps}

A new harp has been installed at 5A-HP-04. This device has commercial ceramic feedthroughs, silicon-carbide wires mounted on a solid ceramic board, and three collecting-field planes. The center collecting field can be energized separately from the two outer plailes so the effects of the different planes can be studied with a view toward possible elimination of the center plane, to simplify construction. New gold-plated connector cards were also installed on the cables for this harp.

\section{Guard-Rings}

The complete system of $\mathrm{H}^{-}$beam guard rings has been connected to the Fast-Protect System and activated.

\section{Line-X Strippers}

Four new strippers have been installed in Line X to replace the old LX-ST-1 through -4 . The old devices consisted of sets of fixed slits which could be rotated through the beam. The new strippers consist of two or four foils, erch of which is run into the $\mathrm{H}^{-}$ beam from the side. The basic module consists of two opposing foils run by separate actuators with position readout. The two foils can form a slit of any opening, centered anywhere across the beam lire. Positive position readout is obtained from the new devices, operation is much faster, and no run-permit interruption is required to change apertures. The LX-ST-1 and -3 have both vertical and horizontal modules mounted to form four-jaw units, as sketched in Fig. IV.1. These two strippers, separated by $15 \mathrm{~m}$, form a pair of adjustable apertures to limit the transverse phase space sent on to Line C. The LX-ST-4 is a two-jaw unit mounted horizontally at the point of highest resolution in 


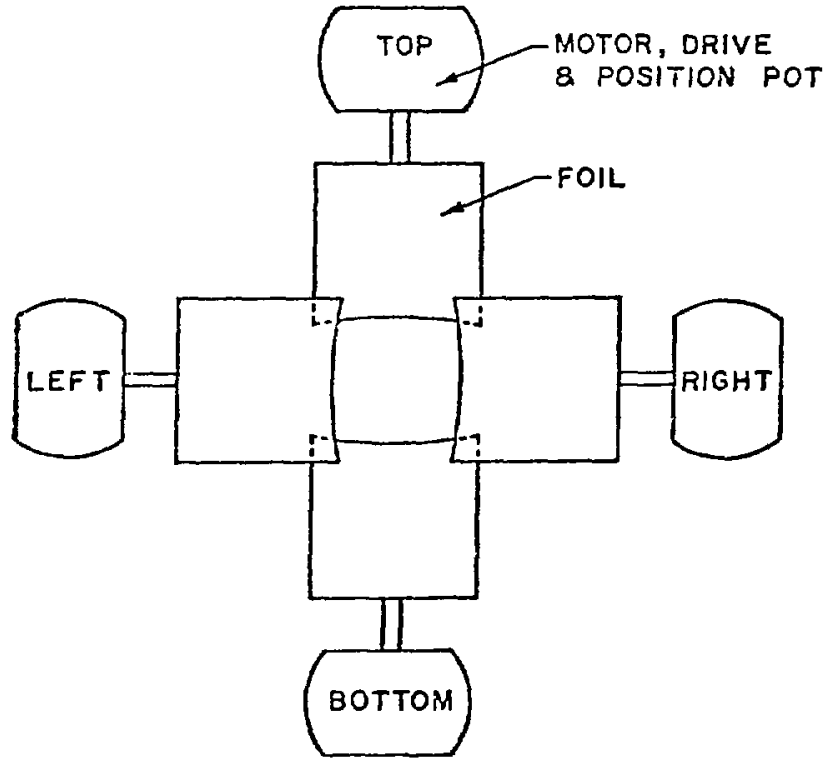

Fig. IV-1.

Neu "four-jaw" stripper-foil arrangement in Line $X$.

Line $X(\sim 0.1 \%)$ to limit the transmitted momentum spread.

\section{Flip-Coil Integrator}

A computer-controlled flip coil is in use to monitor the field in certain bending magnets in the primary beam lines. This instrument and its interface are out-of-date and are a severe reliability problem. As a start toward replacing the device, several techniques for making the necessary measurement were investigated, and a decision was made to go to an integration scheme using dual V/F converters and counters packaged in CAMAC modules. The necessary circuitry was designed and fabricated. Testing on the bench and in the field will take place next quarter.

\section{Electronic Noise Elimination}

The powerline analyzer ordered last quarter was delivered and has been installed in various locations to investigate the correlation between computer crashes and powerline transients. The first conclusion is that the "clean power," a source of ac power supposedly free of noise from heavy electrical equipment, is not so ciean. An effort was initiated to transfer the offending equipment to the "dirty power" circuits. At the Biomedical Facility, where the computer performance is so critical, the analyzer recorded as many as one-hundred $50-\mathrm{V}$ spikes per day. Careful detective work led to a correlation between these spikes and the presence of the Monitor remote-handling equipment working on the A-5 target. To a lesser extent, certain magnetictape transports were cauting noise. The analyzer is scheduled for installation in several other areas next quarter to help diagnose other suspected powerline problems.

The Area A-East RICE (module 66) has been plagued by intermittent noise on the analog data system. The shutdown provided an opportunity to troubleshoot the problem. It was found that the neutral on the clean power system was $6 \Omega$ away from the counterpoise ground. Elsewhere in Area A and the SY, it was discovered that several clean power transformers have their secondary neutral tied to structural members of the building rather than the counterpoise system. These potential sources of noise are scheduled for correction the first week in November.

\section{Actuator Controls}

The controls for three secondary-emission monitors were installed along with controls for operating a cover for the thin target. In anticipation of future needs, these controls were designed with the same circuit as the TV phosphor and could be used for inserting TV phosphors if the need arose.

\section{Interlocks}

The first phase of a major modification to the interlocking scheme for the magnet cooling systems was completed. The magnet power supplies are being interlocked with the "pump on" signals rather than the flow switches, which have proved to be unreliable. Design sketches were completed for a central chassis, located in the SY, which will supply the basic interlocks to the appropriate secondary beamline magnets from the $\mathrm{X} 01, \mathrm{X} 02, \mathrm{X} 03, \mathrm{X} 05, \mathrm{X} 04-6$, 
$\mathrm{X} 07-8$, and $\mathrm{X} 09$ water systems. The first system completed was $\mathrm{X} 05 ; \mathrm{X} 02$ is sclieduled to he next.

Another neutron radiation monitor was installed in Line EP and connected to the Run-Permit system. These beam-area neutron monitors interrupt beam if the preset threshold is exceeded when the area might be occupied; their Run-Permit input is inactive if the area is closed.

Five new thermocouples were installed to monitor temperatures at the new A. 6 window. Three of the thermocouple outputs are connested to the RunPermit system; the other two go to the readout box.

A series input to Run Permit was wired for the Isotope Production stringers flow switch and A-6 windo.. flow.

Five thermocouples were installed to provide monitoring for the new A-5 collimator.

A terminal connected to the control computer was installed in the AMO, $i$ nus providing the AMO staff with information on the status of various systems in the experimental areas.

\section{Magnet Systems}

\section{Power Supplies}

The new interlock relay board. incorporating fault memory, has been installed in 26 power supplies. There are about 20 more that require this modification. All control and metering panels have now been replaced with modified units that give proper control of the reversing switch. The rebuilding program continues on the 2000-A reversing switches, with four more units replaced this quarter. There are 24 more switches that should be replaced and 34 additional switches that will be replaced as time permits. The on/off interlock system for the two special Transrex HRS power supplies has been modified to incorporate the LAMPF-Standard method of control with the latch function coming from the main contactors. The main contactors (which are actually "spring-close, motor-wind breakers") have been repaired to eliminate intermittent operation of the close-command solenoid. Five Acme power supplies, having reversing switches for the magnet current, had their control and metering chassis replaced with an upgraded version of this chassis. Several binary command channels were added so the chassis could be controlled from CCR.
The LB/EP magnet-current data, which currently go into the Line B DVM, were transferred to the Line C DVM, thus concentrating all B-C-EP readout in one DVM, with readout by the $B-C$ CAMAC system.

\section{Direct-Cooled Triplet}

Assembly of the reconfigured target-cell triplet is proceeding, and no difficulties have been encountered in dismantling the magnet or installing the new coils. All the assembly components (except connectors, temperature switches, water insulators, etc.) are on hand and are being tested.

The manufacture of water insulators to our requirements was a major procurement effort in this redesign. An early version of this insulator was shown in Fig. VII-4, LA-6938-PR (September 1977), but had $t$, meet the following specifications:

1) Integral nickel tubing ends, to minimize parts count and eliminate pockets that collect stagnant water.

2) Tubing entry into the ends of the ceramic tube, to give stiffness and ensure the tube forms the electrode. The nickel oxide formed during the operation of the insulators is expected to give reduced corrosion rates compared to the copper electrodes in our present insulator design.

3) Brazes of pure silver for strength and minimal corrosion. Similar insulators produced with soft-soldered attachments have shown inadequate strength for the $300 \mathrm{psi}(20 \mathrm{~atm})$ water system on which they are used.

\section{Components}

\section{Line D}

The completed radiation-hard LD-BM-00 coil sections have been shipped. Upon their arrival at LAMPF, inspection and acceptance tests will be conducted.

The fast-kicker magnet, shown in Fig. IV-2, has been assembled, tested at full power under vacuum, and is now installed in the switchyard. 


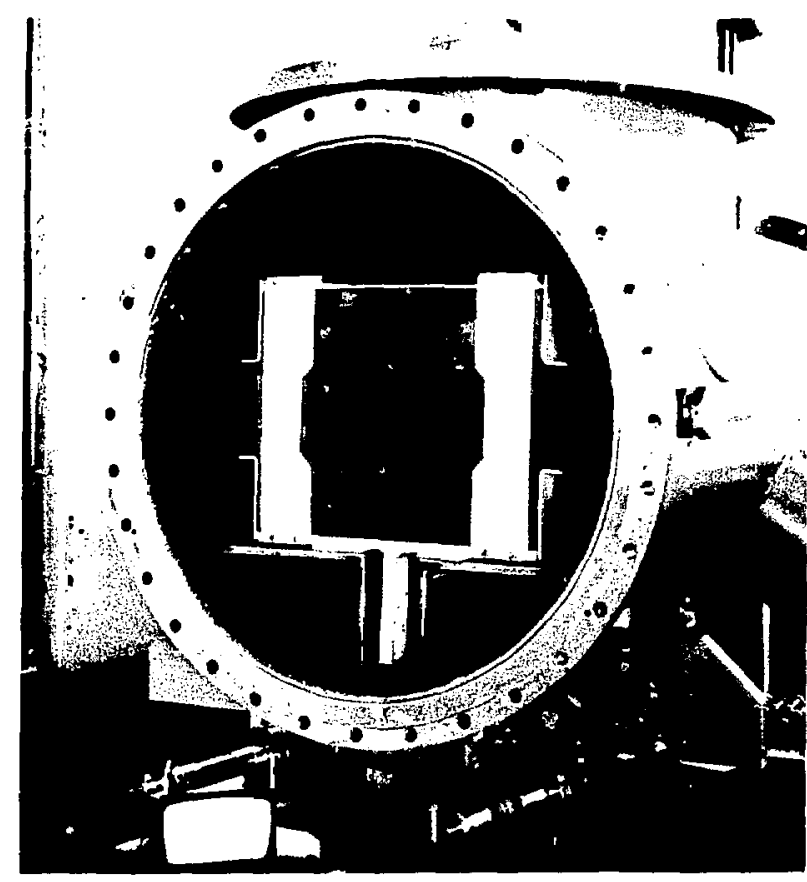

Fig. IV-2.

WNR fast-kicker magnet assembled in its vacuum tank.

An aluminum load resistor tank and a damping resistor tank for the fast kicker have been fabricated this quarter.

\section{Vacuum System}

Extensive modifications were made at the A-3 and A-6 pumping stations, which resulted in a considerable improvement in performance. The roughing time for Line $\mathrm{A}$ has been reduced from $\sim 45$ to $\sim 15$ min. Many of the improvements suggested by Tom Beat, vacuum consultant from Lawrence Livermore Laboratory, are being incorporated.

The control package for the new vacuum pumping system at A-3 was installed and checked for proper operation. The logic to control the 3ASV04 valve between the roughing pump and the pumping manifold was revised so that when the vacuum measured by the manifold thermocouple gauge exceeds $100 \mu$, the valve closes. However, the inverse is not true (i.e., the valve does not open automatically when the vacuum is $>100 \mu$ ); manual action is required.
The controls for several ion pump power supplies were installed in association with the new kicker magnet for Line D.

The turbopump located in the Nuclear Chemistry Cave was replaced by a new unit. In anticipation of a future turbopump in Line EP, a bin of controls and cabling was installed.

\section{Target Cells and Remote Handling}

\section{Targets and Drives}

Two solid-disk graphite wheel targets (similar to the new A-2 design) were installed at A-1. The rim thickness on one of the new targets was changed to $1 \mathrm{~cm}$, to reduce electron production for $\mathrm{LEP}$ experiments.

The new chain-drive spin mechanism has been instailed at A-2. The $\mathrm{P}^{3}$ movable collimator was redesigned in order to eliminate water leakage.

The control system for the A-2 target was modified to run the new A-2 mechanism. Additional wiring was installed so that an indication of target position is available in CCR. The associated draw ings were updated.

A new target-actuator mechanism has been installed at A-5. The new mechanism is driven by a dc motor, thus eliminating many of the problems associated with the hydraulic system in the old mechanism.

\section{New A-5 Target Design}

The stationary pyrolytic graphite target in use at A-5 is cooled by radiation. However, increasing beam currents are creating target temperatures near the level where carbon sublimation becomes a problem. At $2200 \mathrm{~K}$, which will be reached with a beam of $\sim 0.5 \mathrm{~mA}$, the sublimation rate is $\sim 1 \mathrm{~mm}$ per year ( 3000 operating hours). Therefore, the decision was made to build a water-cooled target in order to lower temperatures.

Again, pyrolytic graphite was used for the target material. Figure IV-3 shows an isometric view of the target and its cooling circuit. The a-b planes of the semicircular graphite slabs are perpendicular to the proton beam. In this way, the extremely high heat 


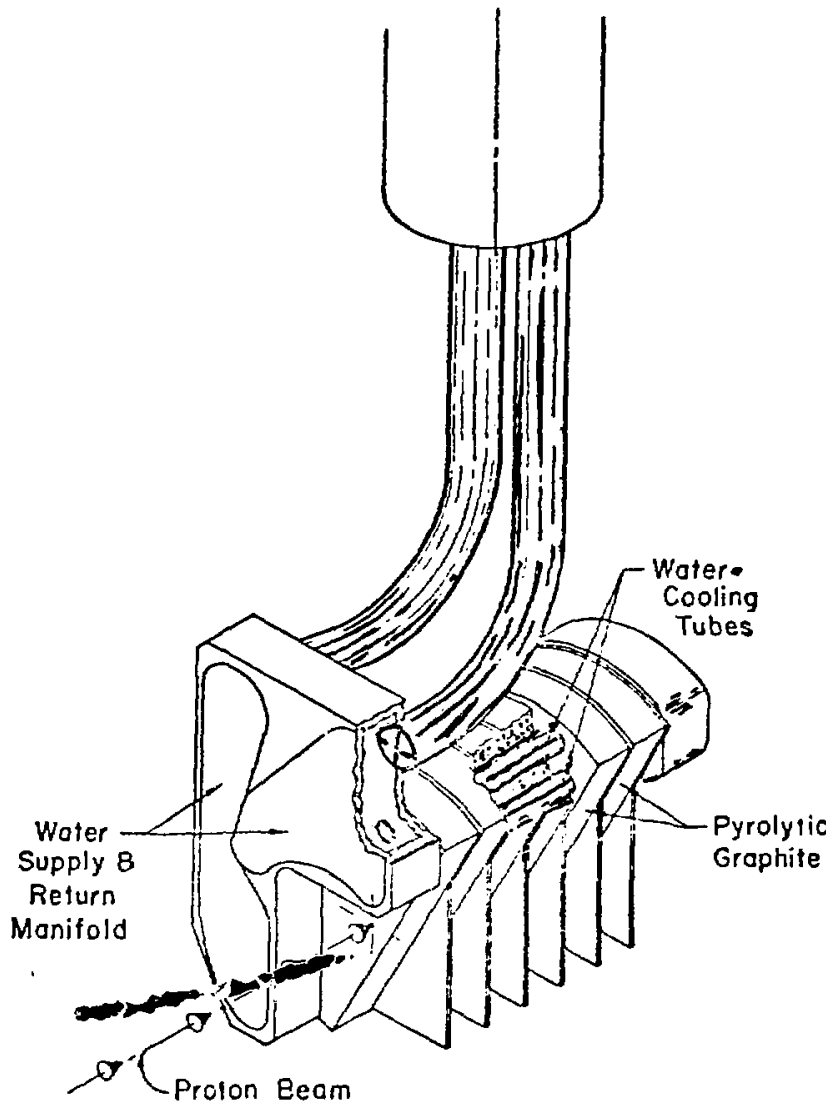

Fig. IV-3.

Water-cooled pion-production target for $A-5$.

conductivity $\left(18 \mathrm{~W} / \mathrm{cm} /{ }^{\circ} \mathrm{C}\right.$ at room temperature and still $4 \mathrm{~W} / \mathrm{cm} /{ }^{\circ} \mathrm{C}$ at $1000^{\circ} \mathrm{C}$ parallel to the a-b planes) is exploited to carry the heat from the beam spot to the peripheral cooling pipes. In addition, a very low thermal expansion coefficient of $1-2 \mathrm{ppm} /{ }^{\circ} \mathrm{C}$, combined with relatively high tensile strength in the direction of the $a-b$ planes, promises good stability against heat stress.

The graphite slabs are brazed onto 6-mm-diam stainless steel cooling tubes, using a technique devised by Lloyd $O$. Lindquist. ${ }^{2}$ The braze was tester. extensively up to heat flux densities of $600 \mathrm{~W} / \mathrm{cm}^{2}$, or about twice that required for $1-\mathrm{mA}$ operation of the new target. Maximum temperatures near the beam spot are expected to stay below $2000 \mathrm{~K}$, with beam currents up to $1 \mathrm{~mA}$.

The slanted plane face of the target permits the experimenter to change the distance between the beam spot and the target surface by moving the target vertically. Thus it is possible to adjust the target for minimum electron production - an important feature for biomedical applications. A set of seven thin vanes, projecting from the target face, provides an alternate thin target with $10 \%$ of the main target thickness, adding flexibility for calibration and dosimetry experinents.

The new target is under construction and is scheduled for installation before startup of Cycle 19.

Graphite-to-stainless steel bonds are being tested in order to determine heat flux capability and the effects of thermal cycling.

Efforts to evaluate proton beam effects on pyrolytic graphite continue. Preliminary results of this investigation are as follow:

1) Polarized light metallography indicates no physical changes in the graphite structure.

2) Autoradiography indicates substantial diffusion of spallation products in the target.

3) X-ray diffraction indicates an annealing effect in the irradiated region.

\section{Remote Handling}

A vacuum leak, located at the first flange downstream from the A-5 target box, was repaired. A new water-colled collimator was installed between the target box and the first flange to protect the flange from excessive heat. It is estimated that this collimator can remove $\sim 27 \mathrm{~kW}$ from the scattered beam. Additional protection is provided by cooling air blown directly on the face of the clamp. Thermocouples were installed to monitor collimator temperature, clamp temperature, and cooling-water inle 'outlet temperatures. Installation of the collimator required more ingenuity and precise remote-handling techniques than any previous remote-handling operation at LAMPF.

The fractured water-cooled A-6 window was replaced with a new window. All components in this new window were ultrasonically and radiographically inspected prior to assembly and testing. Actual stresses were measured with strain gauges during hydrostatic testing.

A separate X02 water circuit was provided for the new window, which incorporates a flow switch (in run-permit) and an air chamber to reduce water hammer. 
To facilitate future remote-handling operations, a new A-6 top collimator was fanricated and in. stalled.

Most of the operations planned for the September-October shutdown were handled in a routine manner; however, some unforeseen operations exceeded current remote-handling equipment capabilities.

Performance of the TeleOperator Systems Corp. servo manipulator greatly exceeded our expectations. Although the force reflection is less sensitive than desired, overall durability and general usefulness far outweighed this deficiency.

\section{REFERENCES}

1. E. W. Hoffman, "Comparison of Phase-Space Measurements in Line A-Direct and Line ASouth, "Los Alamos Scientific Laboratory internal memo MP-13/EWH/T77-05 (July 1977).

2. Lloyd O. Lindquist and Richard Mah. "Graphiteto-Metal Bonding Techniques," Los Alamos Srientific Laboratory report LA-6928-MS (November 1977). 


\section{EXPERIMENTAL AREAS}

\section{New Facilities}

\section{A-East Cover and Crane}

Construction design for Package I of the Area AEast service structure has been completed and is being reviewed. Construction bids are expected to be complete and work started early in 1978. Package I will provide a structural support and the installation of a 30000 -kg bridge crane over Target Stations $A-5$ and A-6 on the main proton beam line.

The new craft building, currently under construction, will be completed in early December. This ruilding will provide an assembly area, space for stock storage, and limited fabrication space for $\mathrm{Zia}$ crafts.

Work has started on the conversion of the present crafts building to a cryogenic laboratory and a nonradioactive assembly area.

\section{Design of 800-MeV-Proton Spin Precessor}

Most efficient use of the polarized beam is obtained hy using all of the accelerated beam in as many simultaneous experiments as possible. The present arrangement of Lines $\mathrm{B}, \mathrm{C}$, and EP allows simultaneous usable beams in all experimental areas if, and only if, the polarization vector is vertical, i.e., perpendicular to the bend plane. If nonvertical polarizaton is required, only one experimental area can receive beam with the desired orientation. In this case, the orientation is adjusted at the Injector $\mathrm{C}$ spin precessor to give the proper results at the experiment.

An $800-\mathrm{MeV}$ proton spin precessor has been designed and preliminary cost estimates compiled. The device is achromatic, tunable, works for a range of energies, and produces no net deflection of the beam while yielding all possible spin orientations at the output from any spin orientation at the input. This precessor could be located in EP to provide compatibility of HRS and EP.

The system requires incident $\mathrm{H}^{-}$and yields $\mathrm{H}^{+}$at its output. It utilizes a property of $\mathrm{H}^{-}$which causes the particle to bend through an angle of opposite sense to the precession of the proton spin while the bend of $\mathrm{H}^{+}$is through an angle with the same sense as the precession of the spin. The complete device consists of two solenoids, three dipoles, and a stripper. The solenoids are the first and last elements and are used for rotations of the spin vector between the vertical and horizontal planes. The dipoles manipulate the beam in the horizontal plane as follows:

The first and third dipoles deflect the beam through an angle $\theta$ while the second dipole deflects the beam through an angle $-2 \theta$ (Fig. $\mathrm{V}-1)$. This results in no net deflection of the beam if the dipoles are equally spaced. The magnetic fields simultaneously cause the polarization vector to rotate through an angle $\phi$ in the horizontal plane where $\phi=-5.585 \gamma^{\theta}$ ( $\gamma=\mathrm{E} / \mathrm{m}$, in standard relativistic notation). For $800-\mathrm{MeV} \mathrm{H}^{-}, \gamma=1.852$, so $\phi=-10.34 \theta$. Thus, a bend of only $8.7^{\circ}$ will rotate the polarization vector by $90^{\circ}$ in the horizontal plane. ${ }^{1}$

LAMPF has dipoles and power supplies available to construct a system to handle all spin orientations for energies between $647 \mathrm{MeV}$ and $800 \mathrm{MeV}$ as well as selected situations below $647 \mathrm{MeV}$. The solenoids are not presently available and would have to be purchased.

\section{Shielding}

Extensive shielding changes were made in the EPICS channel. New blocks were fabricated and installed in the downstream face of the channel, and

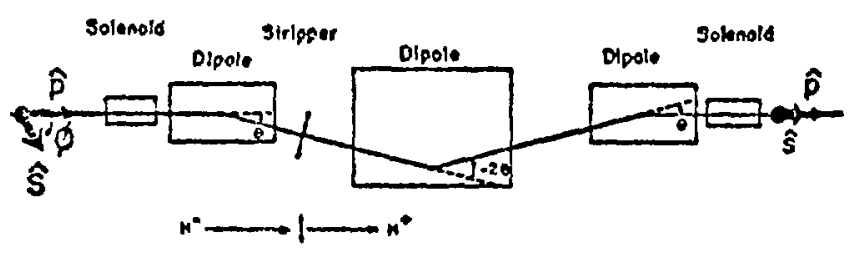

Fig. $V-1$.

Design schematic of the 800-MeV proton spin precessor which can produce any proton spin orientation at the output independent of the input orientation. 
additional shielding was placed at the top of the channel.

Additional shielding was installed near the A-4 air-cooled collimator, reducing the dose rate in the A-4 stubout.

Shielding modifications were made in ISORAD. The handstacked lead brick and concrete blocks covering the $\mathrm{X} 02$ water circuits were replaced with specially fabricated steel blocks.

A $7000-\mathrm{kg}$ slab of steel was removed from the bottom of the A-6 shield door. This slab (a filler piece, bolted to the bottom of the A- 6 shield door), had broken loose at one end and interfered with safe door operation.

Considerable shielding material was removed from Area $B$ and the Line $B$ tunnel as a result of changes in experimental requirements along the EPB line. Extensive changes outside Area $B$ were started to accommodate Exp. 137 on EPB. A new concrete slab was poured in order to allow moving the EPB beam stop farther out along the beam line.

Additional shielding was installed on the HRS frame, to further shield the detectors from beamline radiation. Experiments will be continued on ways to improve this shielding.

Several hundred tons of very gow, flat steel slabs were obtained from excess materials sources; the slabs are a malleable low-carbon steel plates from $22.2-$ to $63.5-\mathrm{mm}$ thick.

\section{Secondary Beam Line Development}

\section{Measurements of $\mathbf{P}^{3}$ Central Momentum}

A method for accurate determination of the central momentum, $\mathrm{P}$, in the $\mathrm{P}^{3}$ channel has been developed using TOF techniques. The basic idea is to measure the TOF of slow particles such as protons, deuterons, tritons, and ${ }^{3} \mathrm{He}$ nuclei relative to fast particles such as pions or electrons. These data, plus a knowledge of the flight path through the channel, determine the $P / z$ of these particles.

Data were collected during low-intensity development runs using stripped and chopped (12.5 MHz) $\mathrm{H}^{-}$beams in Area A. The TOF of all particles was measured relative to the $12.5-\mathrm{MHz}$ timing signal derived from the accelerator $\mathrm{rf}$, and the TOF relative to pions is given by subtraction. In order to reduce rates and produce a well-defined central trajectory, the solid angle and momentum jaws were closed down to $\sim 0.2 \mathrm{msr}$ and $1.5 \%$, respectively.

The chief systematic error was due to shifts in timing with variations in pulse amplitude. At a channel momentum of $450 \mathrm{MeV} / \mathrm{c} / \mathrm{z}$, for example, the doubly charged ${ }^{3} \mathrm{He}$ particles have a $\mathrm{dE} / \mathrm{dX}$ which is 27 times larger than for pions of the same momentum per unit charge. For this situation, one could expect several nanoseconds of timing error from straightforward leading-edge timing. Three other methods were investigated:

a) constant fraction discrimination (Ortec 439).

b) zero crossing, and

c) leading-edge timing where the pulses of each cpecies were separately attenuated (prior to the discriminator) to be about the same am. plitude as the pion pulses.

Method c) gave the least error as judged by two tests:

1. It gave the least variation in the relative $\pi-p$ and $\pi-d$ TOF separation as the timing counter gain was changed from a factor of about 3 below to a factor of 3 above the nominal settings.

2. It gave the most consistent set of values for the channel $\mathrm{P} / \mathrm{z}$ as determined separately from the $\pi-\mathrm{p}, \pi-\mathrm{d}, \pi-\mathrm{T}$, and $\pi{ }^{3} \mathrm{He}$ data.

Measurements were made using method c) for several nominal channel momenta from 200 to $700 \mathrm{MeV} / \mathrm{c}$. The nominal momentum is based on magnetic field measurements of the first bending magnet.

Momenta from TOF data for each of the slower particles relative to pions are tabulated in Table V-I as deviations from the nominal. The consistency of the results obtained from the different particle species is evident in Table V-I. Previous TOF studies, range measurements, and results from magneíic spectrometers looking at elastically scattered pions have also indicated that the central momentum is 1 to $3 \%$ low compared with the nominal.

The cause or causes for the observed deviations are not yet known. One possibility is that the effective length of the first bending magnet is reduced by the steel shielding which is stacked close to it.

Preliminary studies of the reproducibility of the central momentum indicate $0.25 \%$ or better reproducibility for the normal magnet cycling procedure at $450 \mathrm{MeV} / \mathrm{c}$. Studies aimed at finding 
TABLE V-I

\section{CENTRAL MOMENTUM DEVIATIONS FROM NOMINAL}

\begin{tabular}{ccccccc}
$\begin{array}{c}\text { Nominal } \\
\text { Momentum } \\
\mathbf{P}_{\mathbf{0}}(\mathbf{M e V} / \mathbf{c})\end{array}$ & & $\underline{\boldsymbol{\pi}-\mathbf{p}}$ & $\underline{\boldsymbol{\pi}-{ }^{3} \mathrm{He}}$ & $\underline{\boldsymbol{\pi}-\mathbf{d}}$ & $\underline{\boldsymbol{\pi}-\mathbf{T}}$ & Average \\
\cline { 5 - 7 } & & & & & \\
700 & -1.30 & -1.29 & -1.46 & -1.42 & -1.37 \\
600 & -2.29 & -2.27 & -2.27 & -2.00 & -2.21 \\
450 & -2.39 & -1.94 & -1.97 & $*$ & -2.10 \\
350 & -2.45 & -2.11 & -2.05 & $*$ & -2.20 \\
250 & -2.40 & $*$ & $*$ & $*$ & -2.40 \\
200 & -1.40 & $*$ & $*$ & $*$ & -1.40
\end{tabular}

*'The slower particles here had insufficient range to satisfy counter telescope logic.

the minimum amount of magnet cycling for 0.1 $0.2 \%$ reproducibility are planned for the future.

\section{Stopped $\pi^{-}$Ream at SMC-East}

An intense stopping $\pi^{-}$beam is needed for those $\pi$-mesic $x$-ray experiments which use bent-crystal spectrometers. A beam for this purpose has been developed in SMC-East by removing the third bending magnet and converging a $\pi^{-}$beam onto a 7 $\mathrm{cm}$ by $10-\mathrm{cm}$ target $4.5 \mathrm{~m}$ downstream of the last quadrupole in the decay section. Stopping $\pi^{-}$rates of $27 \mathrm{kHz} / \mu \mathrm{A}$ per $\mathrm{g} / \mathrm{cm}^{2}$ of calcium have been measured using lower intensity proton beams. This implies $8.1 \mathrm{Mhz} / \mathrm{g} / \mathrm{cm}^{2}$ of calcium at $300 \mu \mathrm{A}$, which will be sufficient for Exp. 173 if the neutron backgrounds are low enough.

\section{Spectrometer Activities}

\section{Spectrometer Use}

During Cycle 17, HRS was used for Exps. 49 and 311, while EPICS was used for Exp. 14. The resolution at HRS was limited to $300 \mathrm{keV}$ by the need to use a vacuum window at the entrance to the spectrometer, while the use of the small, helium-filled scattering chamber at EPICS contributed to background problems. However, no significant downtime resulting from Group MP-10 equipment was report- ed for either HRS or EPICS during this running cycle.

\section{EPICS - Mechanical}

A circulating oil system for the beam separator high-voltage termination was installed that includes the capability to change oil from outside the shielding. To help keep the oil clean, replaceable filters were also installed on the system. The $n$ w power supply for the separator was installed and tested to $300 \mathrm{kV}$. Since the channel has been open to the air for the whole shutdown period, the outgassing from the various components prevented us from operating the separator at full voltage. As the vacuum improves, the separator voltage will be pushed up higher.

During Cycle 17, a refrigeration system was placed around the scattering chamber to cool the ${ }^{18} \mathrm{O}$ water target. This resulted in condensation seeping into the spectrometer pivot-bearing mount. The pivot was removed and $\sim 300 \mathrm{~cm}^{3}$ water was found in the bearing mount. The bearing mount was modified so that oil in it could be changed without removing it from the spectrometer structure.

The small, old scattering chamber was replaced by a $1.9-\mathrm{m}$-diam chamber. There is no window between channel vacuum and scattering-chamber vacuum, but there is a $0.36-\mathrm{mm}$ Mylar exit window in the scattering chamber and one on the spectrometer entrance port. $\mathrm{A}$ rotating table is installed 
in the scattering chamber. Both the targetpositioning and rotating mechanisms are working.

Two differential-pressure chassis were constructed and installed on the frame. These chassis have readout meters and control the operation of valves between the main line of EPICS and the scattering chamber, and between the scattering chamber target mechanism and the chamber itself.

The frame position readout system was installed and put into operation.

The scattering chamber target controls were completely rewired at the chassis.

An alarm system to indicate power supply trips was installed in the EPICS control racks and documented.

During Cycle 17, the beam plug located near A-1 production target became inoperable. Between $\mathrm{Cy}$ cles 17 and 18, two beam plugs of new design were instailed between EA-BM-03 and -04. Air cylinder drive provides more rapid operating times.

\section{EPICS Magnets}

During the September-October shutdown, alignment of the spectrometer magnets was checked and no misalignment was found.

One EPICS spectrometer dipole was partially re. mapped in situ. The measurement program was completed within the allotted time. Special fixturing had been fabricated beforehand in preparation for this task.

A control system was installed to telp in the setting of magnets and shunts with the gaussmeter. This system ties the controls for two large, remote power supplies and six shunts to the rack where the gaussmeter equipment is located.

\section{EPICS and HRS Detectors}

During the past three months, we experienced sudden increases in the current in the delay-line chambers. We also found that chambers made with gold-plated cathode wire do not suffer from this problem. Subsequently, all of the HRS delay-line chambers and the rear chambers on EPICS were replaced with units made with gold-plated wire cathodes; the EPICS front chambers will be replaced later.

\section{EPICS Multichannel Cerenkov Counter}

A prototype radiator cell with stainless-steel walls and a $0.25-\mathrm{mm}$ stainless-steel entrance window has been successfully tested, and production of all 20 cells should start in November. The most persistent problem has been sealing the top and bottom horizontal members in a massless fashion. This is being solved by counter-bowing the clamping frames on presses at the main LASL shops. The problem of isolating the radiator and prism liquids from their cells has been investigated, and after considering several alternatives (e.g., anodizing techniques, black chrome), Teflon coating was chosen (Thermec Engineering Corp., Anaheim, California). This consists of a black TFE Teflon layer, 0.003-mm thick, applied at $400^{\circ} \mathrm{C}$, and topped with clear PFA Teflon (0.203- to $0.254-\mathrm{mm}$ thick) with the surface made as flat as possible to minimize refleriions.

The design of the photomultiplier light funnels is complete and production of these should also start in November. The design of the prism boxes is still in progress, and production of these should start perhaps a month later. All the mirrors and the radiator-fused silica windows have been received. The fused quartz prism windows should arrive within a month. Of the 60 EMI D2950A photomultipliers ordered, 30 have been received. All (60) positive high-voltage bases have been completed, partly by Group MP-10.

As soon as some radiator cells and light funnels are completed, they will be assembled with one or two of the existing mirrors to provide a pi $+\mathrm{mu}$ monitor in the EPICS channel, to complement the existing ion chambers that count all particles.

\section{HRS Scattering Chamber}

A new rod-transfer mechanism, activated by a LED and photodiode, was installed. In addition, the bellows between the rod-transfer mechanism and HS-QM-01 was changed to a more flexible bellows. These two changes should improve scatteringchamber operation without a window. Also, a larger roughing pump has been installed for pumping down the scattering chamber; this cuts down on pumping time considerably. 
The vacuum shoe test assembly for the scattering chamber's sliding vacuum curtain has been completed. Testing of this unit under vacuum will start as soon as the molded silastic seal is received from CMB-6.

Internal components, including a target and ion chamber, were aligned in the HRS scattering chamber.

Two new motor controls were installed in $\mathrm{CCH}$ for the $\mathrm{x}$ and $\mathrm{y}$ slits to be located in the scattering chamber.

\section{Line C - HRS Controls}

A 15-channel motor-driver CAMAC module and three 15-channel analog multiplexer modules were installed in the $\mathrm{CCH}$. Forty-one analog data channels for the magnet currents from the Line C DVM were remoted to the $\mathrm{CCH}$ CAMAC system. These actions provide an optional mode in which the HRS magnets can be controlled from the $\mathrm{CCH}$ with magnet current data available from the DVM.

\section{Data Systems}

During this quarter, the major portion of the time was spent trying to find ways to improve the eventrate handling capability of the EPICS dataacquisition system. By isolating the most timeconsuming portions of the event-analysis and coding-optimized assembly language calculations, an improvement in data rate from $\sim 11$ to 22 events/s was achieved. The addition, on a trial basis, of a cache memory to the PDP-11/45 resulted in a further $40 \%$ improvement to $\sim 31$ events $/ \mathrm{s}$. Additional software modifications either completed or in progress are expected to increase the rate to $\sim 40$ events $/ \mathrm{s}$ if the cache is retained, or $\sim 28 \mathrm{events} / \mathrm{s}$ if it is not.

In addition, there has recently been an agreement on the use of certain constants at both HRS and EPICS to ensure that code developed for one system may readily be transported to the other if so desired. This is important, since some of the routines developed to improve EPICS data-handling capability will surely do the same for HRS when adopted there. The operating system at both facilities is being upgraded from RSX-11D-VGA to RSX-116-V6.2. This new system has a time-slicing provision that should eliminate the apparent lack of response, so frustrating to the novice user, which occurs when not enough memory is available to load a requested task.

Group MP-10 has received division funding for the purchase of a data-analysis computer system which will consist of a PDP-11/60 processor and a floating-point array processor made by Floating Point Systems, Inc. This combination should allow both HRS and EPJCS data to be analyzed at 3 to 10 times the currently available rate. It is expected that this system will be available in May 1978.

\section{Design of a Low-Resolution Spectrometer (LRS) for HRS}

A low-resolution, second-arm spectrometer for HRS has been studied. This spectrometer, the LRS, is planned for Exp. 258, but its design has been coordinated with some other users. The spectrometer is to sit on the HRS floor with horizontal bend angle, and should have a large acceptance in angle and energy. Additional criteria were to minimize the cost by using existing pool magnets and power supplies. Several similar designs are based upon the 20$\mathrm{cm}$-bore quadrupoles available. Variations are in the choice of $8 \mathrm{Q}-12,-16$, or -32 magnets in doublet or triplet configurations, all followed by an 18D 40 analyzing magnet. The best design, an 8Q16-32-16 triplet with unequal end excitations, has the following parameters:

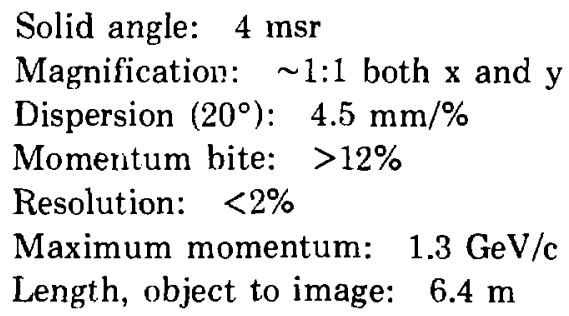

Experiment 258 would require a fixed-angle system (at $\sim 90^{\circ}$ ). The work necessary to install LRS on a movable arm is being evaluated by other users. 


\section{Experiment Support}

\section{Engineering}

The following 11 experiments received engineering and technician support during the quarter, which encompassed a two-month accelerator shutdown:

Exp. $27 I I$ (EPB). The Ames " $\mathrm{C}$ " magnet was moved from ETL to Area B and aligned on a temporary stand. A permanent stand is now being fabricated for this magnet. A scattering chamber was precisely aligned to the beam line.

Exp. 96 (LEP). Extensive design and fabrication efforts were provided. Hardware items completed include the following: target chamber and detector assembly adjustable mounts, beammonitoring ionization chamber, "muon-telescope" beam monitor support, an extension of the LEP vacuum system, and a rectangular window test fixture. The ionization chamber is shown in Fig. V-2.

Exp. 137 (EPB). Criteria changes required redesign of the optical bench to support heavier loads and permit incorporation of larger actuators. Work is proceeding on a scattering chamber of "advanced design" to satisfy the new criteria.

Exp. 181 (LEP). Assembly of the $\pi^{0}$ spectrometer large cart, column assembly, and counterweight hardware has been completed at the ET'L Building. The large cart, complete with column assembly and detector crate attached, is shown in

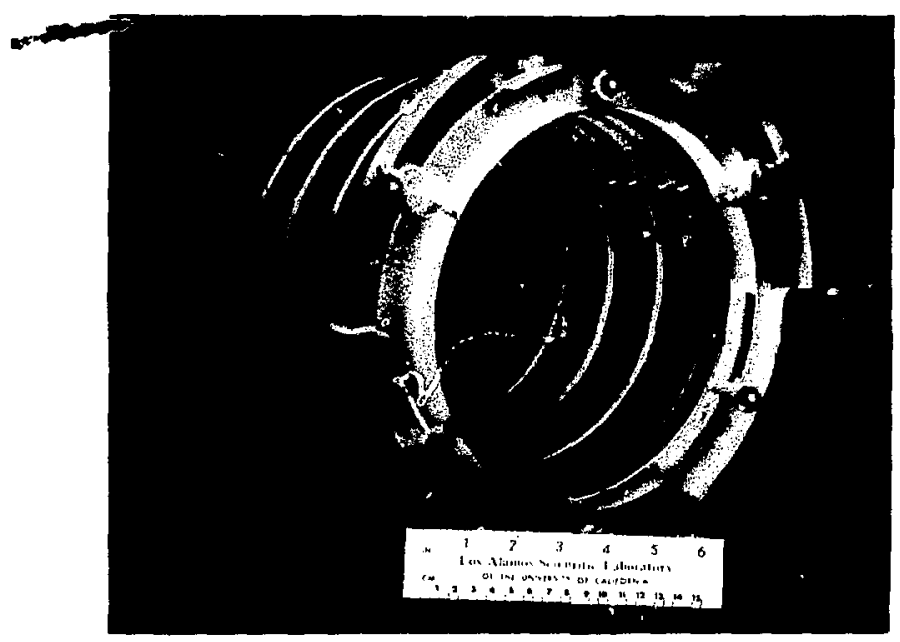

Fig. V-2.

Expertmeru 96 ionization chamber.
Fig. V-3. The cart is designed to operate on casters, as shown, or on air pads that mount on clips under the frame. Associated hardware items completed during this period include a pivot assembly, carriage safety catch, and tension cable assembly. The radius arm assembly is scheduled for completion by mid-November, which will be the last major hardware item required for the spectrometer stand.

A larger cooling system is being fabricated to air cool the PCOS electronic readout units. Converter frames incorporating changes required by the new cooling system are $\sim 75 \%$ complete.

Fourteen wire frames have been fabricated, and wire-winding is complete on five of these frames. Two chambers have been finished and tested, with the total wire chamber effort now $\sim 60 \%$ complete.

Assembly of the second detector crate has started with the inspection, wrapping, and stacking of 15 lead-glass blocks in the crate frame. Two crate rotator assemblies have been installed and tested on the column carriages.

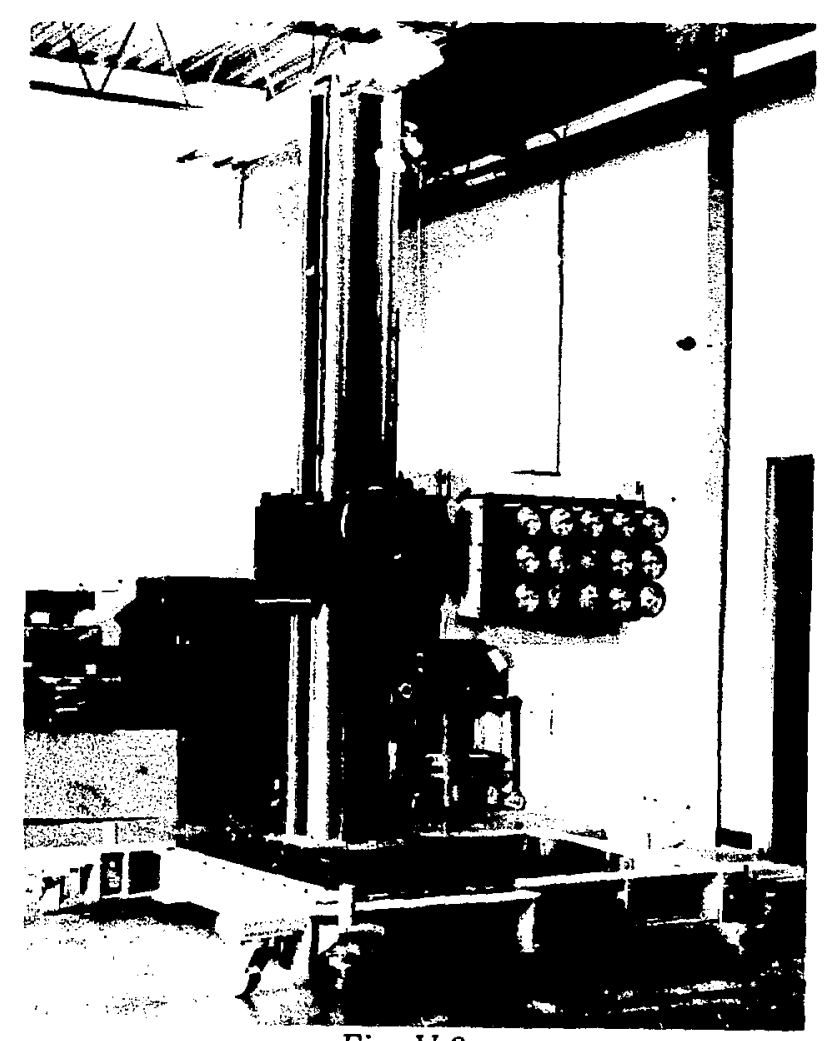

Fig. V-3.

Experiment 181 cart and column assembly with detector crate mounted. 
Design and material procurement has started on a variable density $\mathrm{CH}_{2}$ (polyethylene) target for use in the February 1978 operating cycle.

Exp. $248\left(P^{3}\right)$. Design and drafting are complete for the experiment support structure.

Exp. 258 (HRS). A design study has commenced on a second spectrometer arm for HRS.

Exp. 266/297 (SMC). An air-core, high-field magnet is being designed for the muon spin rotation experiments. Designs for the coil and coil-support structure are complete. Requests for quotations on the magnet conductor have been initiaterl.

Exp. 309/337 $\left(P^{3}\right)$. Design of a stainless-steel scattering chamber and associated hardware, including a remote-driven target ladder, has been completed. Drafting and procurement of hardware are now under way.

Exp. 322 (LEP). A floorplate is being fabricated for installation in the LEP cave that will allow rapid positioning and setup of the "Bicentennial" magnet stand.

Exp. 328 (SMC). Support of this experiment continued with several pieces of apparatus being supplied for collimation and calibration requirements. A Van de Graaff accelerator was installed for the calibration of $\mathrm{NaI}$ crystals.

Exp. 341 (EPB). An activities list for this experiment was prepared and distributed. Major modifications to an existing radius arm issembly and pivot shaft have been completed for this experiment.

General Support. The Ames C magnet has been reworked and the new water-cooled (hollow conductor) coils installed, Fig. V-4. This magnet has been one of the more popular units in the magnet pool, and with these improvements should be more useful and reliable.

Four ion pumps, two $2400-\ell / \mathrm{s}$ and two $600-\ell / \mathrm{s}$, were rubuilt this period. An order was placed for stainless-steel tubing, bellows, and flanges to permit rapid response to the vacuum beam pipe requirements of the experimentalists.

Six experiments were supported by light pipe fabrication as follow: 39 light pipes, 37 scintillators, 60 round rods, and 14 pieces of UVT material, as well as the modification and repair of 31 light pipss.

Assembly activities in the wire-winding shop for this quarter were the following:
Exp. 65/66 1 long and 1 short signal frame

Exp. $96 \quad 3$ hv planes

10 short signal frames

10 long signal frames

Exp. $181 \quad 4$ "Y" signal frames

Exp. 3281 long signal frame

Biomed 4 trays of 1.3-mil carbon monofilament

WNR $1 \mathrm{x}-\mathrm{y}$ frame

\section{Cryogenius}

A reservoir-fed liquid-hydrogen target was constructed and operated for Exp. 328 in Cycle 17. Several improvements have been made; the target will be operated again during Cycle 18 .

A refrigerated liquid-hydrogen target system was built and operated for Exp. 144.

Two liquid-hydrogen target flasks were built and tested to destruction for Exp. 96. Based on the test results, a third target of a modified design has been built. A target vacuum chamber is under construction, and a refrigerator system has been adapted for the target.

Design and drafting are nearly completed for a testing station to measure target flask leak rates.

Modifications to the University of Virginia's ${ }^{3} \mathrm{He}$ target cryostat for Exp. 248 have been performed.

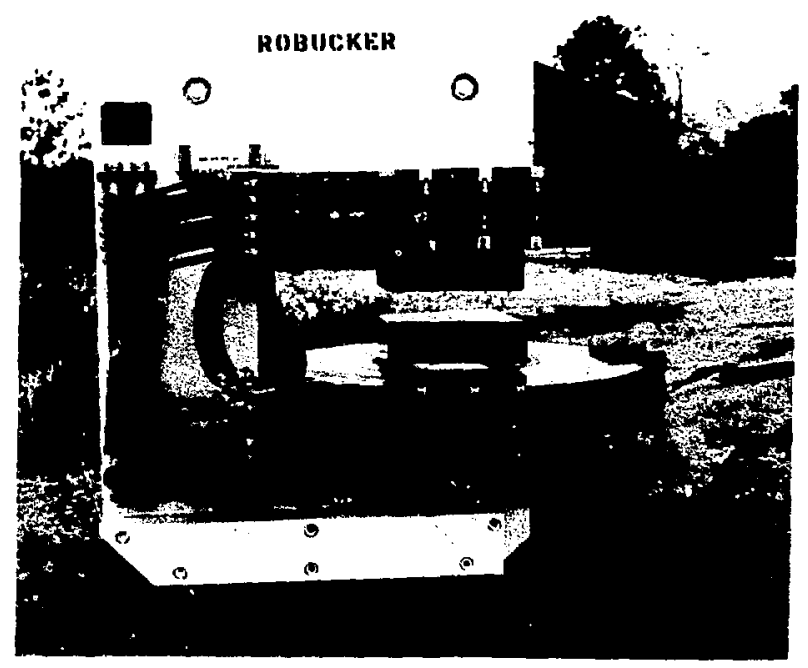

Fig. V-4.

Ames $C$ magnet. 
Substantial maintenance has been done on a Group P-9 cryopump. The maintenance problems seem to be related to the nylon high-pressure piping that was used to isolate the cryopump at a high voltage, and a cold trap has been ordered to correct these problems. This cold trap should prove useful at the LAMPF polarized injector as well, since it has shown similar problems.

Some rough warehouse space is being remodeled, to provide a small cryogenics laboratory. Upon completion, LAMPF will have the capability to perform full tests of liquid-hydrogen targets in a proper laboratory workshop.

Cryogenic Pressure Servo. The specifications for an entirely new cryogenic pressure servo system were prepared. The new design will be based on experience with the previously field-tested system. Progress was made on a block diagram of the new system and on the design of a digital rms power meter which will monitor heater power, and the components were ordered.

Detailed hardware design continued on a microprocessor-based controller which is expecied to reduce the number of manhours required to perform a system cooldown on the LAMPF liquidhydrogen targets.

\section{Polarized Target}

In October, the Saciay HERA superconducting magnet, polarized target cryostat, power supply (which needs some repairs), electronic control rack, protective quenching circuit, and other hardware arrived from France. The only apparent damage that occurred during shipping was a crack in the glass face on one of the electronic meters. Work on the HERA system is being performed in cooperation with J. E. Simmons and J. Boissevain (Group PDOR).

The HERA equipment was obtained with the understanding that new coil covers would be required for the magnet, since the old ones leak. We are proceeding with a design in which the covers will be welded dirently to the coil bobbins. Two consultants have been retained to assist in this effort: an engineer from Group SD-2 and a metallurgist/welding engineer from Group CMB-6. We are attempt- ing to design the covers to allow either an electron beam or a heliarc weld; electron beam welding is preferred because of the reduced heating. There is concern, however, about the tungsten content in the stainless steel of the bobbins. Samples of this special stainless steel will be obtained and welding tests made.

A special frame has been fabricated to support the magnet and provide access to it. Pumping tests on the magnet vacuum jacket indicate that an available $10-\mathrm{cm}$ diffusion pump will be adequate. Based on this, the magnet vacuum system has been designed, and orders have been placed for a cold trap and a mechanical backing pump.

We have designed and submitted for fabrication a special bobbin support jig. This jig will be used to support the bobbins during machining and other operations.

A drawing has been prepared that shows the layout of equipment t? $\mathrm{tat}$ is to be used for a cryogenic test of the polarized target cryostat. The cryostat support stand, the ${ }^{3} \mathrm{He}$ pump line, and the cryostat vacuum system have been designed. Fffort is continuing on the design of the remaining items necessary for such a test.

The HERA equipment represents the core of a longitudinally polarized target. However, the system cannot be used without additional equip. ment in the form of NMR and microwave electronics, pumping systems, and special gas-handling equipment. Such equipment has been under development. During the past quarter, a $4-\ell$ activated charcoal trap was designed and built for the ${ }^{3} \mathrm{He}$ gas-handling system. This system is now complete, except for a special transfer pump that has been ordered. The ${ }^{3} \mathrm{He}$ pump system has been leakchecked and the pumps have been operated. Completion of the system awaits delivery of the Alcatel MIV-3000 pump that was ordered earlier this year. At a later time, we expect to replace the LeyboldHeraeus Roots pumps with pumps that will reduce air leakage and contamination.

The components for the microwave system have been assembled and mounted. In order to complete this system, we have ordered a special water-cooled heat sink for the klystron.

Design and assembly of the electronic control rack for the polarized target have started. A multiplexing system for digital readout of the flow meters has been designed and fabrication started. An order has 
been placed for a new signal generator for the NMR system.

\section{Data Acquisition}

Experimental Area Computers. Progress continued on the long-range program to implement the recommendations of two summer studies dealing with the computing needs at LAMPF.

The PDP-11/70 computer for data analysis arrived and was installed. This system will go into production as soor as a problem involving the Unibus and the Diva disk is resolved. The added computing capacity will relieve the load on the Terminal computer.

The PDP-11/34 computer for the test channel arrived. As soon as the trailer to house it is available, the system will be insualled and turned over to the experimenters.

A procurement action was started to upgrade the PDP-11/45 Terminal computer to a PDP-11/70. The present PDP-11/45 will be moved to $\mathrm{P}^{3}$-East to replace the PDP-11/34. The latter will be moved to CCR to become a more powerful interface between the SEL- 840 control computer and the RIU. When completed, these actions will increase the dataanalysis capacity at LAMPF, improve the dataacquisition capability at $\mathrm{P}^{3}$-East, and decrease the load on the accelerator control computer.

The PDP-11/34 CPU obtained last quarter from C Division in exchange for a PDP-11/20 is being upgraded to a standard LAMPF data-acquisition system. This upgrade involves the addition of more memory and a floating-point hardware unit.

A digital time-of-day clock was installed on the Terminal computer, and software was written to set the system time and date from the output of the clock, eliminating manual entry at system startup.

The staff continued to contribute to a Laboratorywide program to evaluate peripheral equipment for the PDP-11 computers at LASL. Work began on a standard for an electrostatic printer/plotter.

Data-Acquisition Software. The integration of Program $Q$ with the new Version 6.2 of RSX-11D was completed. Several experiments will be using this new release of $Q$ during the next production cy- cle. One of the principal advantages of the new versions is that it provides all of the capabilities of $\mathrm{Q}$ and RSX-11D for the PDP-11/34s.

Progress continued toward the goal of a complete set of documents describing Program Q. The first of several manuals, " $Q$ and the Analyzer Task," LA6884-M, was received from the printers. Two other manuals are in for printing, a third is in editing, and a fifth manual was written in preliminary form and assigned a report number for reference purposes.

Work was completed on a floating-point emulator for RSX-11D. This program simulates the FP-11 floating-point hardware available on the more powerful PDP-11 models. A paper describing this work is in preparation.

Additional effort was spent in editing an appendix to the standard for CAMAC subroutines being prepared by the NIM-CAMAC Software Working Group. This document is now complete and will he published soon as a TID document.

A new version of the NOVA Call Basic software featuring magnetic-tape subroutines was installed on the magnet-mapping computer.

Computer Maintenance. The accelerator shutdown provided the maintenance teams with an opportunity to work on a backlog of jobs that accumulate during a production cycle. All of the computers received preventive maintenance, and various pieces of broken equipmen. and pc cards were repaired.

As an outgrowth of the MBD summer study, all of the engineering cilanges (ECO) that have been developed for this device were collected and validated. including a recent one which deskews the timing on the trailing edge of the Unibus address lines and was submitted by a user at the University of Saskatchewan. Using this collection as a guide, a project was initiated to bring all of the MBDs up to the same ECO level. Three MBDs have been completed to date. Each one takes about 2-3 weeks to upgrade. MBDTST, a program for testing the MBD in the single address mode, was modified to work on all memory sizes up to the $4 \mathrm{k}$ maximum.

Plarger office/lab trailer was obtained to provide more space for the growing computer maintenance operation. The trailer will be installed next quarter. 
LAMPF Electronics Equipment Pool (LEEP). The LEEP was granted a supplementary allocation of funds during the final quarter of FY-78. These funds were used to purchase: $10 \mathrm{NIM}$ dual-gate generators, 10 NIM octal discriminators, 10 NIM coincidence units, and 10 NIM bins.

A list of needed equipment was prepared for submission to the I.SEP conmittee.

Three 6-V-dc, 45-A power supplies were donated to LEEP; each can add a $6-\mathrm{V}$ capability to several NIM bins.

Two of eight new Event Trigger modules were tested using programs developed for the computerbased test stand.

The ability of the LEEP personnel to respond to an experimenter's need for equipment depends on the accuracy of the information in the LEEP data base. To audit this information, a major inventory was initiated. Each experimenter was asked to verify that the LEEP equipment listed on a printout from the data base was still in his possession. About half have responded.

Data-Analysis Summer Study. A final report of the summer study dealing with the problems of data analysis was completed (LA-7034-MS). It will be available for presentation to the users at the Elevent h Annual LAMPF Users Group Meeting in November. The recommendations of the study group are as follows:

1. Sufficient computing power should be obtained to meet the estimated data-analysis load of LAMPF. This may be accomplished with the following computer systems:

a. The PDP-11/70 purchased in FY-77 represents the first increment.

b. In FY-78, sufficient equi, yment should be purchased to upgrade two computers - one to an enhanced PDP-11/60 for use at HRS/EPICS and another to a normal PDP. 11/70. A third PDP-11/70 or a new 32-bit computer should be ol uered.

c. In FY-79, the new 32-bit computer should be enhanced with additional peripherals and a second matching system should be ordered.

d. A midicomputer, or its equivalent in computing power and physical characteristics, should be ordered as soon as possible, but arrival time should be no later than the first of FY-80.

2. A building should be constructed to house the midicomputer and the other data-analysis computer systems. This building should be located near the experimental area and should include work space for users, offices for computer support personnel, and storage space for magnetic tapes and supplies.

3. The staff of personnel who support the dataacquisition and analysis effort - programmers, consultants, operators, and data analysts - should be increased by a minimum of $t w o$ additional people in $\mathrm{FY}-78$, three in $\mathrm{FY}$ 79 , and by two more in FY -80.

Preliminary steps were taken to include these recommendations into the $\mathrm{m}$. nagement planning for upcoming years.

Experiment Reviews and Debriefing. The practice of debriefing experimenters as they approach the end of their allotment of beam time continued. Reviews were held for Exps. 29/54, 5, 49, 328. 144. $82,14,341,286$, and $27-$ II. The feedback that comes from these sessions is translated into a worklist from which assignments are made as personnel become available. The following are typical of the responses made this quarter:

1. The recommendations for new electronic modules were referred to the LEEP committee.

2. Some of the suggestions for improving the soft ware for the data-acquisition computers were scheduled for attention.

3. In response to complaints about the LEMOtype coax connectors used with CAMAC inoduies. Kings Electronics (the major supplier of these connectors) was contacted. and the company is now evaluating samples that were sent to them.

CAMAC Auxiliary Controller. A jroposal was made to design and fabricate a prototype CAMi.AC Auxiliary Controller for control purposes on the experimental beam channels. The controller, based upon a design completed last year by Don Machen of MP-1 on leave at The Daresbury Laboratory in 
the U.K., will utilize an LSl-11/02 microcomputer board and will reside completely in a CAMAC crate for increased reliability.

The Auxiliary Controller concept is permitted by the new CAMAC specification on "Multiple Controllers in a CAMAC Crate," TID-26617, and allows several processors to share the same CAMAC Dataway in addition to the conventional Branch or Serial Highway.

Secondary beam line control is generally implemented by local devices, with some channels coupled to the beam line computer via the CAMAC interface system. The Auxiliary Controller will implement channel setup and general control functions independently of the channel computer. However, the channel computer can access all interface devices available to the Auxiliary, thus providing the degree of coupling necessary for the experimenters.
High-Speed Data-Acquisition Device. The Auxiliary Controller noted above can also support multiple bipolar microprogramming facilities capable of executing subroutines at a 100 . ns/instruction cycle time. Such facilities can be optimized for either fast register transfer (from a C.AMAC device to a memory) or for a special set of romputations.

A study is being made into the feasibility of using an Auxiliary Controller, with a microprogramming facility, to generate secondary triggers for a proposed expcriment at LAMPF. The triggers would involve track finding and verification following a primary trigger, thereby filtering the event information and reducing the overall magnetic-tape load for the experiment. If the results of the study are positive, a formal proposal for the device will be developed next quarter. 


\section{RESEARCH}

\section{Results of Experiments}

\section{Breakup of Few-Nucleon Systems and Nuclei (Exp. 42)}

(Univ. of California at Santa Barbara, LASL, Univ. of Southern California)

The breakup reactions ${ }^{m} \operatorname{Li}(p, p y) X, m=6,7 ; y=$ $p, d, t$ have been studied with the major emphasis placed on measurement at angles corresponding to quasi-free scattering from constituents of the lithium target nuclei. The scattered protons were momentum-analyzed in a magnetic spectrometer while the energies of the conjugate particles $(y)$ were measured in an eight-element intrinsic germanium telescope.

Preliminary analyses. reported last quarter, focused on data reduction of the ${ }^{8} \mathrm{Li}(\mathrm{p}, \mathrm{pd})$ measurements at two quasi-free angle pairs. These analyses revealed the presence of two peaks in the missing energy spectra corresponding to residual systems of ${ }^{4} \mathrm{He}$ in its ground state and ${ }^{4} \mathrm{He}$ in a state of $\sim 30$ $\mathrm{MeV}$ excitation. The momentum distributions extracted for recoil ${ }^{4} \mathrm{He}$ (g.s.) particles were Gaussian in shape with FWHM of $\sim 100 \mathrm{MeV} / \mathrm{c}$ for both the $30.0^{\circ}-62.29^{\circ}$ and the $40.0^{\circ}-54.13^{\circ}$ angle pairs.

During this quarter, the efforts were devoted to the reconstruction of the acceptance function of this experiment (i.e., the available three-budy phase spacel using the Monte Carlo technique.

The programming task is now essentially completed. It is anticipated that the large sample event generation and the extraction of the deuteron Fermi momentum distribution in ${ }^{8} \mathrm{Li}$ nuclei would commence shortly.

\section{Muonic X-Ray Studies of Tissues and Tissue- Like Materials (Exp. 100)}

(LASL, Technical Univ. of Munich, Washington State Univ., Univ. of Mississippi)

Description of Experiment. Our purpose was to investigate the feasibility of doing muonic $x$-ray analyses (MXA) for determining the relative concentrations of major and minor elements in tissue and for detecting changes in concentrations of selected elements in the body. We measured yields of muonic $x$ rays from the various elements in a material sample. Figure VI-1 shows a typical muonic $x$-ray spectrum from a tissue sample. The tota! Lyman series yield from a given element is a measure of the relative concentration of that element.

The first set of measurements were on animal tissues (muscle, fat, liver, bone, and blood) and on samples of tissue-equivalent plastic and tissueequivalent liquid. The muonic $x$-ray measurements of carbon, nitrogen, and oxygen concentrations in the muscle, fat, and liver samples were close to the concentrations obtained with conventional techniques, but some unexpected discrepancies were noted. We attributed the differences to sampling variations encountered in taking small specimens for conventional analysis from the large and somewhat inhomogeneous tissue masses used in MXA. Results from muonic $\mathbf{x}$-ray studies of homogeneous materials of well-known composition. e.g.. blood. plastic, and tissue-equivalent liquid, gave very close agreement with the known elemental concentrations, and provided further evidence that sampliiug problems are the source of the discrepancies found with the tissue samples. These results indicate that, for the purpose of aralyzing the average composition of relatively large samples, MXA possesses a distinct advantage over those techniques requiring very small samples.

The results of the analysis of the plastic and tissue-equivalent liquid data are given in Table VII. Table VI-II contains the results of both conventional and muonic $x$-ray analyses of the various tissue samples.

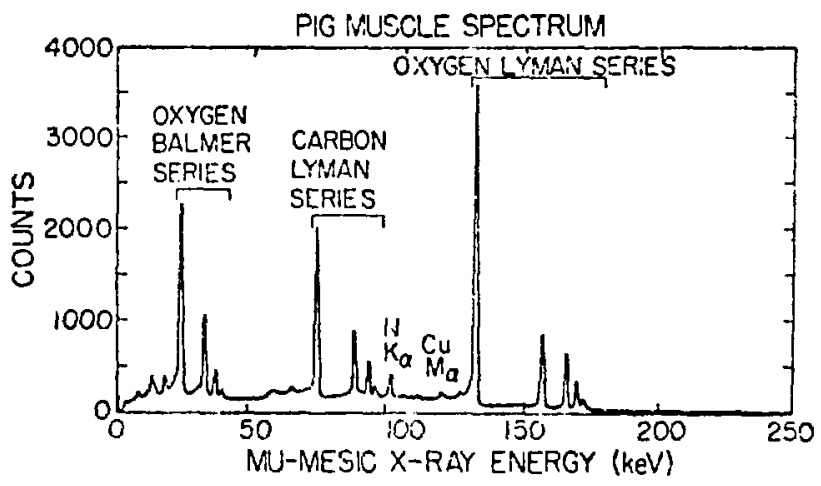

Fig. VI-1.

Typical muonic $x$-ray spectrum from pig muscle tissue. 


\section{TABLE VI-I}

\section{RELATIVE MUONIC X-RAY YIELDS (\%)}

$\begin{array}{lcccr}\text { Material } & & \text { Element } & \begin{array}{c}\text { Prediction Based on } \\ \text { Known Composition }\end{array} & \text { Measured } \\ \text { Shonka } & & & & \\ \text { A-150 Plastic } & \mathrm{N} & & 87.7 \pm 0.4 & 87.8 \pm 0.8 \\ & \mathrm{O} & 3.7 \pm 0.1 & 3.7 \pm 0.2 \\ & \mathrm{~F} & 6.4 \pm 0.7 & 6.5 \pm 0.3 \\ & \mathrm{Ca} & 1.1 \pm 0.1 & 1.1 \pm 0.2 \\ \text { Tissue- } & \mathrm{C} & & 1.1 \pm 0.1 & 1.0 \pm 0.7 \\ \text { Equivalent } & \mathrm{N} & & 5.0 & \\ \text { Liquid } & \mathrm{O} & 80.3 & 3.9 \pm 0.2 \\ & & & & 81.9 \pm 0.6\end{array}$

In order to gain a better estimate of the detection limits for relatively low $Z$ elements, we measured the x-ray yields from dilute solutions of $\mathrm{NaF}, \mathrm{KCl}$, $\mathrm{CuSO}_{4}$, and $\mathrm{AgNO}_{3}$.

Whole-body calcium measurements can be done with neutron activation analysis, while bone mineral levels in the arm, for example, can be determined by gamma transmission measurements. However, since localized areas such as the head or spine are somewhat inaccessible with these established techniques, MXA might be useful for analysis in such regions of the body. We obtained two samples of plastic whose calcium and phosphorous concentrations were not identical but approximated those of a typical bone. The ratio of the yield of calcium $x$ rays from one sample to the yield from the other sample and the ratio of phosphorous yields were $1.26 \pm 0.10$ and $1.61 \pm 0.27$, respectively, while theory predicts ratios of 1.30 and 1.37. This illustrates that MXA is sensitive to relatively small changes in bone mineral content.

Two samples each of normal and cirrhotic human liver were studied. For comparison between normal and abnormal samples, we calculated the ratios of the total yields of the oxygen Lyman series $x$ rays to the yields of the carbon Lyman series. These results are shown in Table VI-III, where a significant and consistent difference is shown between normal and abnormal tissue. Application of background corrections would tend to increase the difference in the ratios.

\section{Conclusions}

Sensitivity and Selectivity. It is important to emphasize that MXA is not a trace element analysis technique. MXA must be looked upon as a major element analysis technique. Detection limits, with the experimental configuration that we've used so far, start at $\sim 0.1 \%$ by weight for beryllium ( $Z=4$ ) and increase gradually to $\sim 5 \%$ for silver $(Z=47$ ) and $10 \%$ by weight for uranium $(Z=92)$. With larger multidetector systems, detection limits could range from $0.01 \%$ to $1 \%$ over this range of $Z$. One advantage of MXA is that the sensitivity varies smoothly and slowly through the periodic chart whereas neutron activation analysis, for example, can have orders-of-magnitude differences between the sensitivities to different elements. This can create problems when measuring the concentrations of elements present in roughly equal amounts but which have widely different activation cross sections. MXA does not suffer from this drawback.

MXA is excellent for establishing unambiguous identification of an element. For example, all elements have unique $K_{\alpha}$ energies with no chance of confusing one for another. (The $\mathrm{K}_{\alpha}$ line of one element may overlap some other line from a different element, but since there are usually several distinguishing lines from each element, an unambiguous identification is almost always possible.) Neutron activation analysis can also suffer from ambiguity in assigning a parent element to the 
TABLE VI-II

\section{CARBON, NITROGEN, AND OXYGEN CONTENT (PERCENT BY WEIGHT) OF ANIMAL TISSUE SAMPLES}

\begin{tabular}{|c|c|c|c|c|c|c|c|}
\hline \multirow[b]{2}{*}{ Sample Description } & & \multicolumn{2}{|c|}{ Carbon } & \multicolumn{2}{|c|}{ Nitrogen } & \multicolumn{2}{|c|}{ Oxygen } \\
\hline & & Content & Error & Content & Error & Content & Error \\
\hline Pig muscle (1-1), & $\mathbf{M}$ & 15.45 & \pm 0.85 & 3.47 & \pm 0.47 & 81.08 & \pm 0.96 \\
\hline Liquid nitrogen frozen & $\mathrm{C}$ & 16.17 & & 3.15 & & 80.68 & \\
\hline Pig muscle (1-1) & $\mathbf{M}$ & 13.58 & 0.69 & 2.80 & 0.31 & 83.62 & 0.75 \\
\hline Thawed & $\mathrm{C}$ & 16.17 & & 3.15 & & 80.68 & \\
\hline Pig muscle (1-1), & $\mathbf{M}$ & 18.05 & 0.76 & 3.27 & 0.27 & 78.68 & 0.80 \\
\hline Refrozen & $\mathrm{C}$ & 16.17 & & 3.15 & & 80.68 & \\
\hline Pig muscle (1-2), & $\mathbf{M}$ & 18.07 & 0.67 & 3.31 & 0.28 & 78.62 & 0.72 \\
\hline Liquid nitrogen frozen & $\mathrm{C}$ & 13.65 & & 3.30 & & 83.05 & \\
\hline Pig muscle (1-3), & $\mathbf{M}$ & 14.46 & 1.05 & 2.93 & 0.41 & 82.61 & 1.12 \\
\hline Liquid nitrogen frozen & $\mathrm{C}$ & & & & & & \\
\hline Pig muscle (2-1) & M & 10.21 & 0.85 & 4.25 & 0.46 & 85.29 & 0.96 \\
\hline Dry ice frozen & $\mathrm{C}$ & 16.78 & & 3.36 & & 79.86 & \\
\hline Pig muscle (2-2), & $\mathbf{M}$ & 14.65 & 0.89 & 4.86 & 0.41 & 80.49 & 0.98 \\
\hline Freezer frozen & $\mathrm{C}$ & 25.23 & & 3.63 & & 71.14 & \\
\hline Pig muscle (2-3), & $\mathbf{M}$ & 16.04 & 0.84 & 4.48 & 0.45 & 79.48 & 0.95 \\
\hline Liquid nitrogen frozen & $\mathrm{C}$ & 12.90 & & 3.55 & & 83.55 & \\
\hline Pig muscle (2-4), & M & 15.01 & 1.02 & 4.08 & 0.50 & 80.91 & 1.13 \\
\hline Formalin preserved & $\mathrm{C}$ & & & & & & \\
\hline Pig fat (1-1), & $\mathbf{M}$ & 52.96 & 1.05 & 1.81 & 0.21 & 45.22 & 1.05 \\
\hline Liquid nitrogen frozen & $\mathrm{C}$ & 47.43 & & 2.21 & & 50.37 & \\
\hline Pig liver (1-1), & M & 14.81 & 0.64 & 3.42 & 0.29 & 81.77 & 0.70 \\
\hline Liquid nitrogen frozen & $\mathrm{C}$ & & & & & & \\
\hline Bovine muscle (WA-1), & M & 8.56 & 1.12 & 4.29 & 0.59 & 87.15 & 1.27 \\
\hline Liquid nitrogen frozen & $\mathrm{C}$ & 12.95 & & 3.72 & & 83.32 & \\
\hline Bovine liver (WA-7), & M & 13.75 & 0.70 & 1.72 & 0.33 & 84.52 & 0.77 \\
\hline Freezer frozen & $\mathrm{C}$ & & & & & & \\
\hline Bovine liver (WA-12), & M & 12.27 & 0.74 & 3.03 & 0.42 & 84.70 & 0.84 \\
\hline Liquid nitrogen frozen & $\mathrm{C}$ & 13.36 & & 2.70 & & 83.94 & \\
\hline Dog liver, & $\mathbf{M}$ & 20.36 & 0.90 & 4.07 & 0.27 & 75.58 & 0.94 \\
\hline Freezer frozen & $\mathrm{C}$ & & & & & & \\
\hline Dog blood & $\mathbf{M}$ & 9.03 & 0.49 & 3.14 & $0.2 \mathrm{k}$ & 87.83 & 0.55 \\
\hline & $\mathrm{C}^{\mathrm{a}}$ & 10.75 & & 3.23 & & 86.02 & \\
\hline Bovine bone, & $\mathbf{M}^{\mathrm{b}}$ & 40.68 & 1.07 & 3.37 & 0.20 & 47.83 & 1.10 \\
\hline Freezer frozen & $\mathrm{C}$ & & & & & & \\
\hline
\end{tabular}

"Human blond composition taken from "Report of Task Group on Reference Man: Gross and Elemental Content of Reference Man." ICRP Report 23 (1974), Chap. 2.

bPlus: phosphorus $=1.90 \pm 0.29 \%$, calcium $=6.22 \pm 0.93 \%$. Normalized so that the sum of all five components is $100 \%$.

$\mathrm{C}=$ content as determined by conventional chemical analysis; $\mathrm{M}=$ content as determined by muonic $\mathrm{x}$-ray analysis. The data have been normalized so that the sum is $100 \%$. The absolute sums are typically of the order of $85-90 \%$, the remainder are mostly hydrogen. 
TABLE VI-III

\section{CALCULATED RATIOS OF TOTAL YIELDS OF OXYGEN LYMAN SERIES X RAYS TO YIELDS OF CARBON LYMAN SERIES}

\begin{tabular}{lll} 
- & Normal Liver & Cirrhotic Liver \\
\cline { 2 - 3 }$\# 1$ & $1.24 \pm 0.01$ & $1.16 \pm 0.01$ \\
$\# 2$ & $1.24 \pm 0.01$ & $1.17 \pm 0.01$
\end{tabular}

radionuclide produced in the neutron exposures. In summary, then, MXA is sensitive to essentially all elements in the periodic chart except hydrogen, and the sensitivity varies smoothly.

Sample Size and Preparation. Considerations of count rate and beain size lead to the conclusion that MXA can conveniently be applied to biological samples as small as $1 \mathrm{~g}$ and as large as $\sim 500 \mathrm{~g}$. Little sample preparation is necessary since a sample can simply be suspended in air or in an inert atmosphere in the muon beam. For very precise quantitative studies it would be desirable to have samples of regular and uniform shapes.

Doses and Count Times. The dose given to a biological sample during a muon wraditation is rather small. To accumulate $10^{4}$ counts in the oxygen spectrum from a $200 \mathrm{-g}$ sample of typical soft tissue, the dose required would be $<1 \mathrm{mrad}$, with a count time of $\sim 1 \mathrm{~min}$. For smaller element concentrations, say $0.4 \%$ potassium in a $200 \mathrm{~g}$ sample, $10^{3}$ counts would require a 0.4 -rad dose with a count time of $\sim 1.3 \mathrm{~h}$, in a counter arrangement similar to ours.

Applications. There are several circumstances in which MXA has advantages over other methods of tissue elemental analysis for in vivo studies.

1. In cases where very small tissue specimens are ordinarily taken for analysis, the problem of obtaining results that are representative or a larger volume of tissue is considerable. MXA obviates this problem by giving compositions averaged over several cubic centimeters of tissue.
2. With proper choice of muon beam size and energy, one can interrogate selected regions of the interior of the body.

3. Surgical intervention is unnecessary with MXA.

4. MXA results in very small radiation doses to the body.

\section{Spallation Yield Distributions from Fast Pion Interactions with Complex Nuclei (Exp. 103) (LASL, BNL)}

Cross sections have been determined by $\mathrm{Ge}(\mathrm{Li}) \gamma$ ray spectrometry for $\sim 20 \quad \gamma$-emitting spallation products resulting from $50-\mathrm{MeV} \pi^{+}$and $\pi^{-}$reactions with copper. The irradiations were performed at the LEP channel and they supplement the previous irradiations we conducted at the $\mathrm{P}^{3}$ channel at 100 , 190 , and $350 \mathrm{MeV} .{ }^{1}$ The $50-\mathrm{MeV}$ data are given in Table VI-IV. We also include cross sections calculated by Ginocchio with his latest version of the VEGAS-ISOBAR intranuclear cascade code for pions; his calculations include the ISOBAR plus the DFF evaporation codes (see Sec. VII, p. 58). At 190 and $350 \mathrm{MeV}$, the calculations reproduced the experimental cross sections fairly well. However, at $100 \mathrm{MeV}$ and now at $50 \mathrm{MeV}$, the calculations considerably underestimate the cross sections.

In Fig. VI-2, the sums of the measured and calculated cross sections are plotted at four energies. Although the sums represent but $\sim 25 \%$ of the total inelastic cross sections, they demonstrate the influence of the $(3,3)$ resonance for spallation products even from medium-mass nuclei. The measurements show the $\pi^{-}$cross section sums to be somewhat larger than for $\pi^{+}$, while the calculations predict the converse.

\section{Fragmentation of Gold by Pions and Protons (Exp. 118) \\ (Purdue Univ., LASL)}

The process of fragmentation (emission of light fragments) in high-energy reactions is not yet completely understood. Two distinct mechanisms are thought to be possible: localized energy transfer (LET) and the development of a coherent cascade 
TABLE VI-IV

\section{CROSS SECTIONS FOR REACTION PRODUCTS FROM $50-\mathrm{MeV} \pi^{ \pm}$ON COPPER}

\begin{tabular}{|c|c|}
\hline \multicolumn{2}{|c|}{ Nuclide } \\
\hline $9.2-\mathrm{h}$ & ${ }^{62} \mathrm{Zn}$ \\
\hline 3.4-h & ${ }^{\epsilon 1} \mathrm{Cu}$ \\
\hline 36.0-h & ${ }^{57} \mathrm{Ni}$ \\
\hline $18.0-\mathrm{h}$ & ${ }^{55} \mathrm{Co}$ \\
\hline $78.0-\mathrm{d}$ & ${ }^{58} \mathrm{Co}$ \\
\hline $271.0-\mathrm{d}$ & ${ }^{57} \mathrm{Co}$ \\
\hline $71.0-\mathrm{d}$ & ${ }^{58} \mathrm{Co}$ \\
\hline 1.6-h & ${ }^{61} \mathrm{Co}$ \\
\hline 8.3-h & ${ }^{52} \mathrm{Fe}$ \\
\hline $45.0-\mathrm{d}$ & ${ }^{59} \mathrm{Fe}$ \\
\hline $5.6-d$ & ${ }^{52} \mathrm{Mn}$ \\
\hline $312.0-\mathrm{d}$ & ${ }^{54} \mathrm{Mn}$ \\
\hline 2.6-h & ${ }^{58} \mathrm{Mn}$ \\
\hline $23.0-\mathrm{h}$ & ${ }^{48} \mathrm{Cr}$ \\
\hline $42.0-\mathrm{m}$ & ${ }^{49} \mathrm{Cr}$ \\
\hline $28.0-\mathrm{d}$ & ${ }^{51} \mathrm{Cr}$ \\
\hline $16.0-\mathrm{d}$ & ${ }^{48} \mathrm{~V}$ \\
\hline $59.0-\mathrm{h}$ & ${ }^{44} \mathrm{Sc}^{\mathrm{m}}$ \\
\hline $84.0-\mathrm{d}$ & ${ }^{48} \mathrm{Sc}$ \\
\hline $3.4-\mathrm{d}$ & ${ }^{47} \mathrm{Sc}$ \\
\hline $44.0-\mathrm{h}$ & ${ }^{48} \mathrm{Sc}$ \\
\hline 22.0-h & ${ }^{43} \mathrm{~K}$ \\
\hline
\end{tabular}

\section{Yield}

Type

$\underline{1}$

$1.8 \pm 0.3$

$10.3 \pm 0.9$

$2.35 \pm 0.41$

$2.97 \pm 0.40$

$13.3 \pm 3.0$

$44.0 \pm 4.0$

$35.0 \pm 4.0$

$1.49 \pm 0.45$

$0.23 \pm 0.04$

$\mathrm{C}=1$

$\mathrm{C}=\mathrm{I}$

C

I

C

C

C

C

C

I

I

I
I

$6.1 \pm 0.3$

$23.5 \pm 1.7$

$2.26 \pm 0.24$

$0.17 \pm 0.07$

$1.46 \pm 0.34$

$18.1 \pm 5.4$

$2.93 \pm 0.25$

$0.39 \pm 0.08$

$0.39 \pm 0.09$

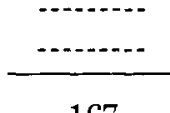

167

\begin{tabular}{c} 
Calculated \\
\hline $2.7 \pm 0.8$ \\
$19.6 \pm 2.2$ \\
$3.6 \pm 1.0$ \\
$5.2 \pm 1.1$ \\
$14.0 \pm 0.2$ \\
$19.0 \pm 3.0$ \\
$12.6 \pm 1.8$ \\
$0.58 \pm 0.38$ \\
$0.63 \pm 0.40$ \\
$0.23 \pm 0.23$ \\
$12.5 \pm 1.7$ \\
$10.7 \pm 1.6$ \\
$1.16 \pm 0.54$ \\
$0.23 \pm 0.24$ \\
$2.5 \pm 0.8$ \\
$12.8 \pm 1.8$ \\
$5.0 \pm 1.1$ \\
$0.25 \pm 0.25$ \\
$0.43 \pm 0.33$ \\
$0.35 \pm 0.30$ \\
$\cdots \cdots$ \\
$\cdots \cdots$ \\
$-\cdots$ \\
-124 \\
\end{tabular}

\begin{tabular}{cl} 
Measured \\
\hline
\end{tabular}

Calculated

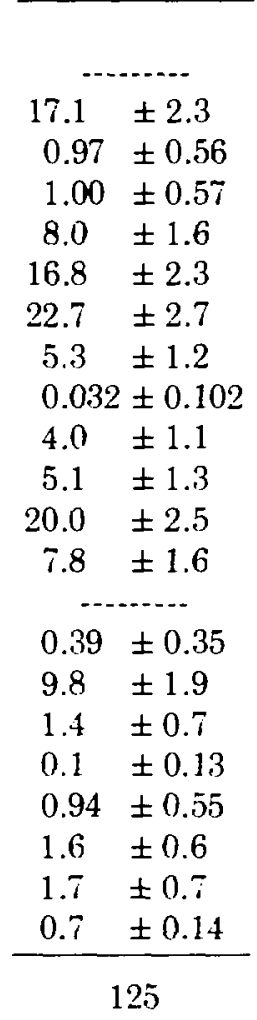

(CC). The LET process involves the formation of a nuclear hot spot, presumably, though not necessarily, due to pion production and reabsorption. The $\mathrm{CC}$ mechanism involves the development of a coherent cascade in which a number of nucleons travel towards the nuclear surface with very low relative momentum and so can coalesce into a fragment. Both mechanisms lead to fragment emission prior to equilibration, and in both cases the interplay between surface tension and Coulomb repulsion forces determines the eventual outcome of such interactions. It is clear, however, that the nature of the initial state is quite different in the two cases.

The study of fragment emission in reactions induced by pions appears to be a potentially useful way to determine the relative importance of these two mechanisms. Because of the likelihood of ab- sorption, pions are known to transfer mcre energy to a target nucleus than protons of the same kinetic energy. On the other hand, pions are much less effective than protons in initiating a coherent cascade because the momentum of LAMPF energy pions is much smaller than that of protons with the same energy. To a first approximation it may thus be concluded that if fragment emission is more probable for pions than protons of the same kinetic energy, then the LET process predominates in proton fragmentation. If the reverse is true, the $\mathrm{CC}$ process is dominant.

We have attempted to answer this question by measuring the excitation functions for the formation of ${ }^{54} \mathrm{Na}$, a typical light fragment, from gold in reactions induced by protons and negative and positive pions. A preliminary report of this work has been 


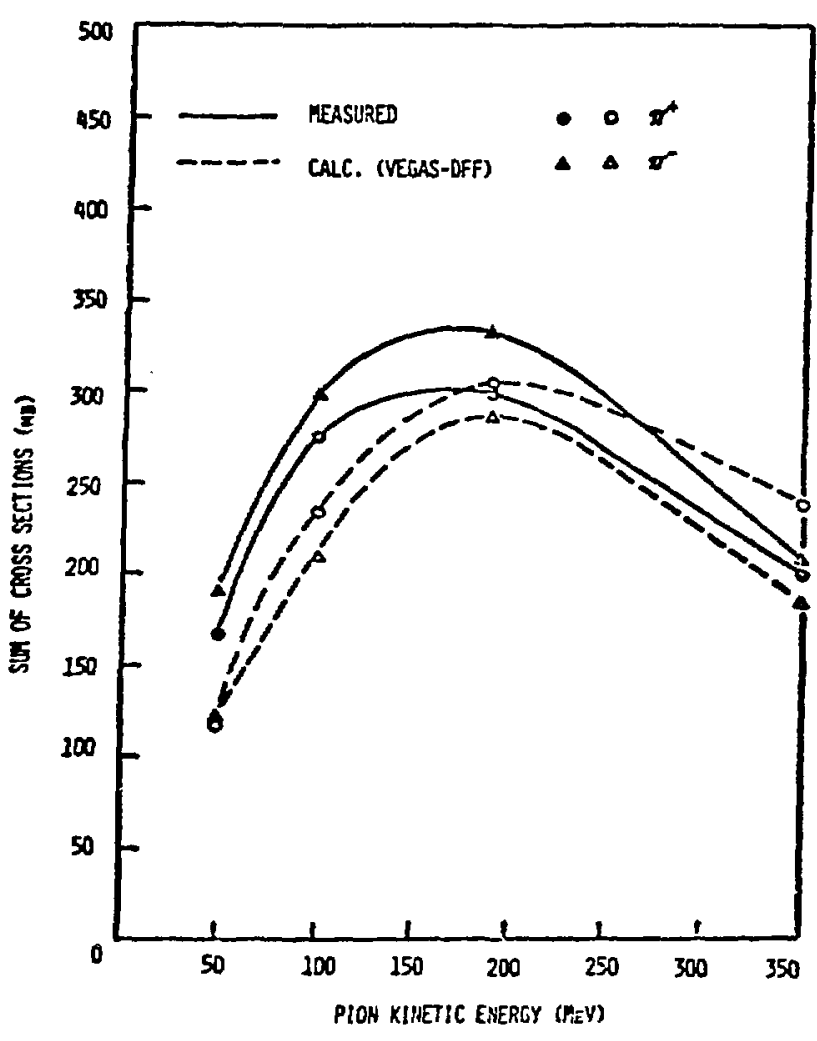

Fig. VI-2.

Comparison of the sums of the measured $\gamma$ emitting spallation products from the interactions of fast pions with copper with the sums calculated using the VEGAS-ISOBAR intranuclear cascade plus DFF evaporation codes.

published. ${ }^{2}$ The proton irradiations were performed in the nuclear chemistry irradiation facility in Area $B$ and in the switchyard area. The pion bombardments were carried out in the $\mathrm{P}^{\mathrm{s}}$ channel. The excitation functions are shown in Fig. VI-3. It is seen that at a given energy the cross sections are highest for $\pi^{+}$and lowest for protons. While this result suggests that the LET process is of primary importance, the rather large and somewhat surprising difference between the $\pi^{+}$and $\pi^{-}$cross sections suggests that a more detailed analysis of the results must be performed before any conclusions can be drawn from the data.

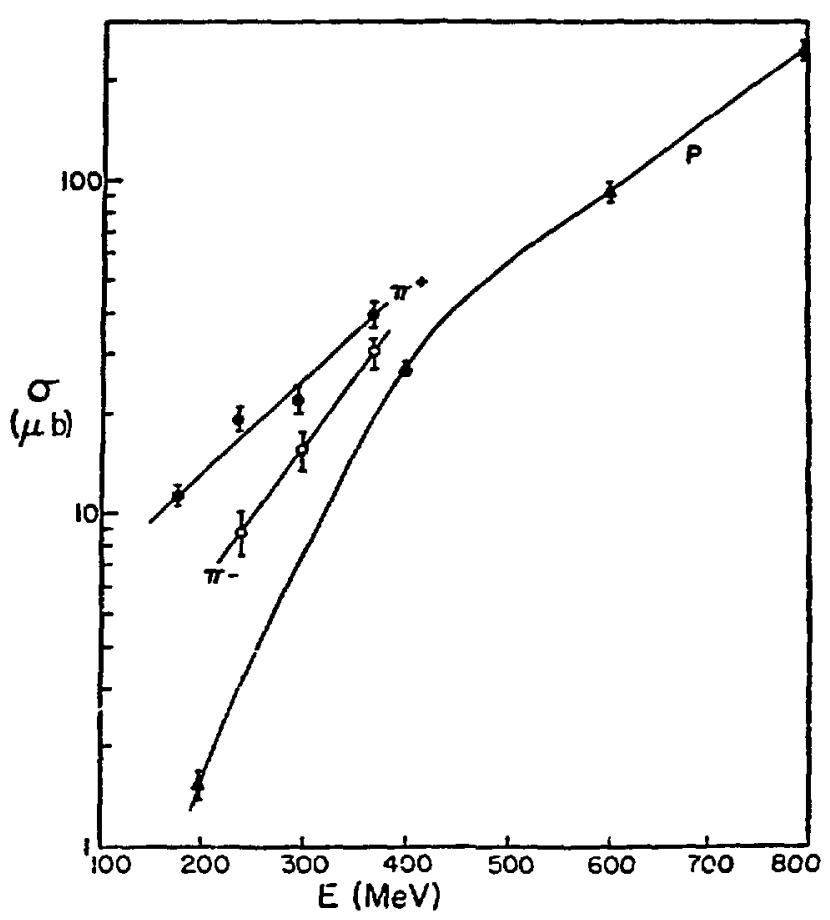

Fig. VI-3.

Excitation functions for the formation of ${ }^{24} \mathrm{Na}$ from gold by protons, $\pi^{+}$, and $\pi^{-}$.

\section{Cross Sections of Simple Nuclear Reactions In- duced by $\pi$-Mesons (Exp. 119) \\ (ANL, LASL)}

The int?uence of the nuclear environment on the cross sections for $\left(\pi^{ \pm}, \pi^{ \pm} n\right)$ and $\left(\pi^{ \pm}, \pi^{ \pm} p\right)$ reactions in the vicinity of the $\Delta(3,3)$ resonance is being investigated. To date, ree 1 lts have been obtained for the reactions ${ }^{197} \mathrm{Au}\left(\pi^{ \pm}, \pi^{ \pm} \mathrm{n}\right)^{196} \mathrm{Au}$ and ${ }^{25} \mathrm{Mg}\left(\pi^{ \pm}, \pi^{ \pm} \mathrm{p}\right){ }^{24} \mathrm{Na}$ over the energy range 100 $300 \mathrm{MeV}$. These data are shown in Fig. VI-4, where they are compared with corresponding data for the free pion-nucleon and ${ }^{12} \mathrm{C}\left(\pi^{ \pm}, \pi^{ \pm} n\right){ }^{11} \mathrm{C}$ reactions. The nuclear reaction cross sections exhibit a considerable mass dependence. Although the ${ }^{25} \mathrm{Mg}\left(\pi^{+}, \pi^{+} \mathrm{p}\right){ }^{24} \mathrm{Na}$ data are essentially identical to the analogous ${ }^{12} \mathrm{C}\left(\pi^{-}, \pi^{-} n\right)^{11} \mathrm{C}$ data, somewhat less energy dependence is seen in the ${ }^{25} \mathrm{Mg}\left(\pi^{-}, \pi^{-} \mathrm{p}\right)^{24} \mathrm{Na}$ data compared with the ${ }^{12} \mathrm{C}\left(\pi^{+}, \pi^{+} n\right)^{11} \mathrm{C}$ data. In the case of ${ }^{187} \mathrm{Au}$, the cross sections are considerably larger (the data in the figure have been reduced by a 


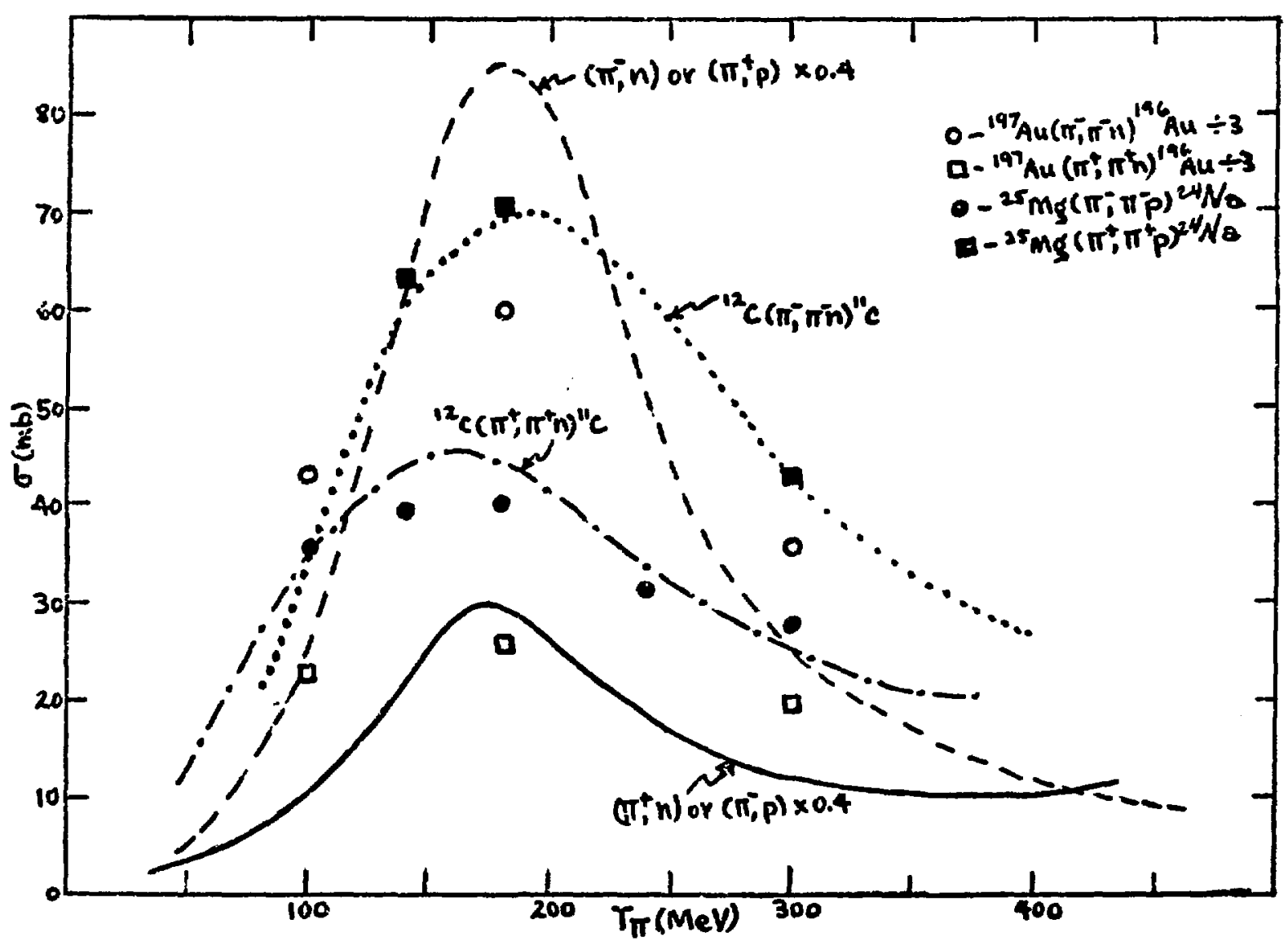

Fig. VI-4.

Cross sections for $\left(\pi^{ \pm}, \pi^{ \pm} n\right)$ or $\left(\pi^{ \pm}, \pi^{ \pm} p\right)$ reactions.

factor of 3 for plotting) and show even less energy dependence in the resonance region. This is particularly true for the ${ }^{187} \mathrm{Au}\left(\pi^{+}, \pi^{+} n\right)^{196} \mathrm{Au}$ data for which the effect of the $\Delta(3,3)$ resonance is barely in evidence.

The ratios of the cross sections for the $\pi^{-}$and $\pi^{+}$. induced reactions in ${ }^{25} \mathrm{Mg}$ and ${ }^{197} \mathrm{Au}$ are compared with the analogous reactions in ${ }^{12} \mathrm{C}$ and for the free pion-nucleon interactions in Fig. VI-5. A satisfactory theoretical interpretation of these data has not yet been given.

Additional studies are under way for the reactions on ${ }^{58} \mathrm{Ni}$ and ${ }^{142} \mathrm{Ce}$ in which both the $\left(\pi^{ \pm}, \pi^{ \pm} n\right)$ and $\left(\pi^{ \pm}, \pi^{ \pm} p\right)$ reactions are examined in the same nucleus.

\section{Proton-Scattering Survey Experiment}

(Exp. 139)

(LASL, Northwestern Univ., Univ. of Minnesota, Univ. of Oregon, Univ. of Texas, UCLA)

Elastic data for $800 \mathrm{MeV}$ protons on $\mathrm{H},{ }^{12.13} \mathrm{C}$. ${ }^{58,60,62,64} \mathrm{Ni}$, and ${ }^{208} \mathrm{~Pb}$ have now been analyzed, as well as some selected inelastic data including the $\left(4.44,2^{+}\right),\left(7.67,0^{2}\right):\left(9.64 .3^{-}\right)$, and $\left(14.1 .4^{+}\right)$levels in ${ }^{12} \mathrm{C}$; the $\left.\left(3.09,1^{1 / 2}\right),\left(3.68,3 / 2^{-}\right),\left(6.68,{ }^{5}\right)^{+}\right)$, and $\left(7.55,52^{-}\right)$levels in ${ }^{13} \mathrm{C}$, and more than 10 levels in ${ }^{58} \mathrm{Ni}$. The resolution obtained for the latter data was $<200 \mathrm{keV}$ full-width at tenth-maximum (FWTM) so that most of the levels were fully resolved (Fig. VI-6). Angular distributions for the $0_{1}^{+}, 2_{1}^{+}$, and $4_{1}^{+}$ levels in ${ }^{88} \mathrm{Ni}$ are shown in Fig. VI-7. At these 


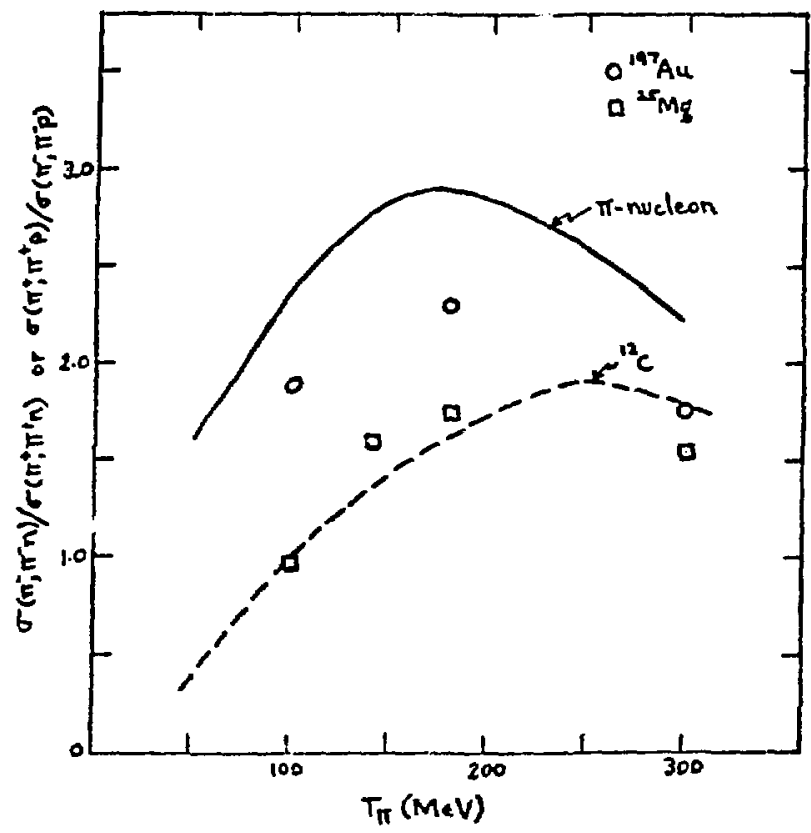

Fig. VI-5.

Ratios of $\pi-$ and $\pi^{+}$-induced reactions.

forward directions, the peaks are characteristic of the angular momentum transfer rather than the Blair phase rule that they asymptotically approach at momentum transfers $q>400 \mathrm{MeV} / \mathrm{c}$ or so. The elastic scattering shows considerable sensitivity to specific details of the neutron and proton densities. Figure Vl-s illustrates this point by comparing proton scattering on ${ }^{58} \mathrm{Ni}$ over a range of energies from $40-1000 \mathrm{MeV}$. While the strength of the effective interaction is generally increasing as the incident energy is reduced, the range is not, i.e., the minima for the 800 - and $1000-\mathrm{MeV}$ data agree to better than $\pm 0.02 \mathrm{~F}$. This is further borne out by doing conventional fits to the data, i.e., by varying the neutron density parameters for carbon, nickel, and lead. These provide rms neutron radii that agree to within $\pm 0.01 \mathrm{~F}$ with the preuictions of densitydependent Hartree-Fock calculations. The detailed distributions do not agree, but show differences in the interior region of the nucleus. The inelastic data show evidence for the spin-flip quadrupole mode of excitation of the nucleus.
Pion Total Cross-Section Measurements with an Oriented ${ }^{165} \mathrm{Ho}$ Target (Exp. 164)

(Lockheed Palo Alto Research Lab., National Bureau of Stantards, California Institute of Tech., LASL, New Mexico State Univ., Univ. of Basel, Univ. of Montana, Univ. of Washington, Univ. of Wisconsin)

This is the final report on LAMPF Exp. 164, the measurement of pion total cross-section differences for aligned and unaligned ${ }^{165} \mathrm{Ho}$. A full paper on this experiment has been published [Phys. Rev. C16, 2367 (1977)].

Pion beams offer unique advantages as probes for the extraction of nuclear structure information. The appropriate choice of pion energy and charge state makes it possible to explore selectively either the neutron or proton distribution in the target nucleus. This feature has been exploited in several recent experiments to measure differences in the average radii of the neutron and proton distributions. ${ }^{3,4}$ The pion mean-free path in nuclear matter is generally short, reaching a minimum of $\sim 0.5 \mathrm{fm}$ at the peak of the $(3,3)$ resonance, which makes the pion effective as a probe of the nuclear surface region. This effectiveness is further enhanced by the fact that, at the peak of the resonance, calculated cross sections tend to be independent of the particular model employed. Jacob and Kofoed-Hansen ${ }^{5}$ have pointed out that, in the case of nuclei with large deformations, pion-scattering experiments from aligned targets should answer some fundamental questions about the deformed structure; e.g., what are the details of the nuclear matter distribution responsible for the observed multipole moments, and do the neutron and proton distributions differ significantly?

These considerations have motivated a study of the total cross section for the interaction of pions with aligned ${ }^{185} \mathrm{Ho}$ nuclei in the $(3,3)$ resonance region. Holmium-165 is a favorite choice for oriented target experiments since it is monoisotopic and its large hyperfine interaction energy ${ }^{6}$ makes orientation by thermal equilibrium techniques possible at relatively high temperatures. 


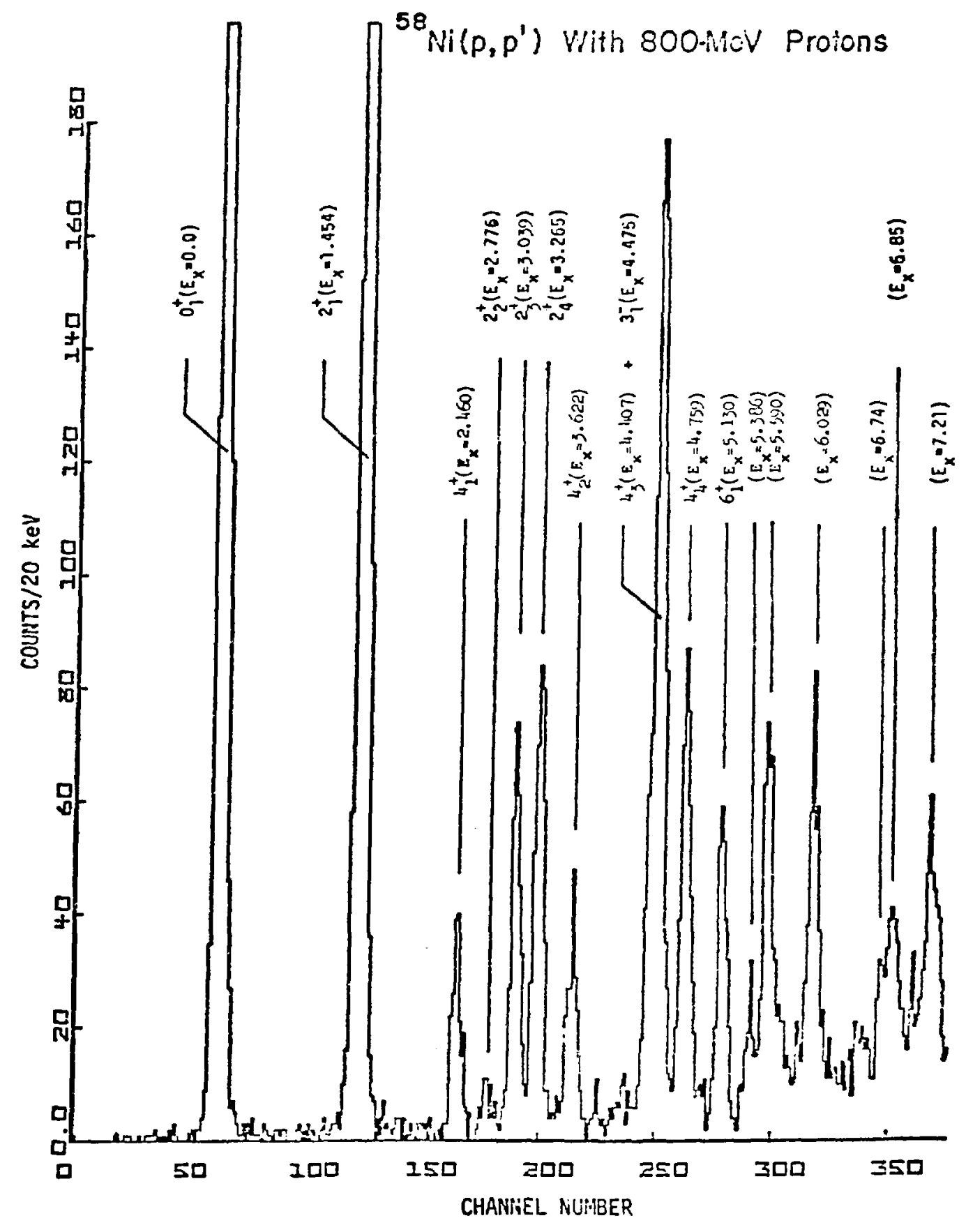

Fig. VI-6.

Spectrometer for ${ }^{88} \mathrm{Ni}\left(p, p^{\prime}\right)$ taken with $800-\mathrm{MeV}$ incident protons at a mean laboratory angle $\theta_{\mathrm{lab}}=18^{\circ}$. The FWTM is $\angle 200 \mathrm{keV}$, so that many levels are resolvable. The assignmer $i$ of spins, parities, and excitation energies was made using previously available information in addition to the data obtained in this experiment. The energies are expected to be accurate to $\pm 4 \mathrm{keV}$. 


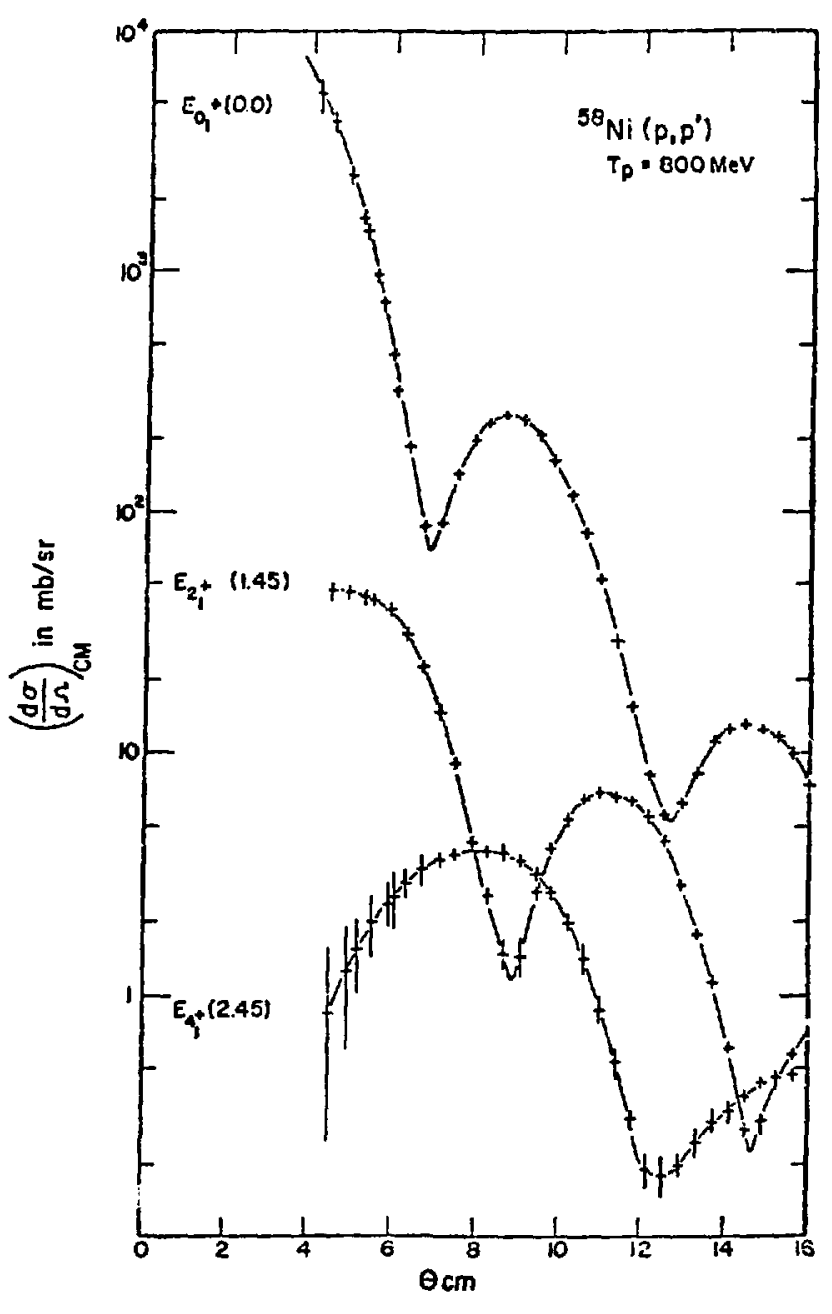

Fig. VT-7.

Angular distributions for the first three excited states in ${ }^{58} \mathrm{Ni}$ showing peaks characteristic of the different angular momentum transfers.

The experimental technique 4 is essentially the same as that desrribed for measuring total crosssection differences in the calcium isotopes. It is aimed at reducing the systematic errors in the measurements of total cross sections between targets, in this case for aligned and unaligned ${ }^{185} \mathrm{Ho}$.

The removal cross-section differences $\Delta \sigma(\Omega)$ for the interaction of $\pi^{+}$and $\pi^{-}$with aligned ${ }^{185} \mathrm{Ho}$ was measured at $E_{\pi}=115,165$, and $240 \mathrm{MeV}$ employing a single crystal holmium target with a nuclear alignment $B_{2}=-0.44$. The range of $\Omega$ was from 0.05 to $0.55 \mathrm{sr}$. The data on $\Delta \sigma(\Omega)$, the difference between the aligned and unaligned cross sections, were compared to the predictions of a coupled-channels op-

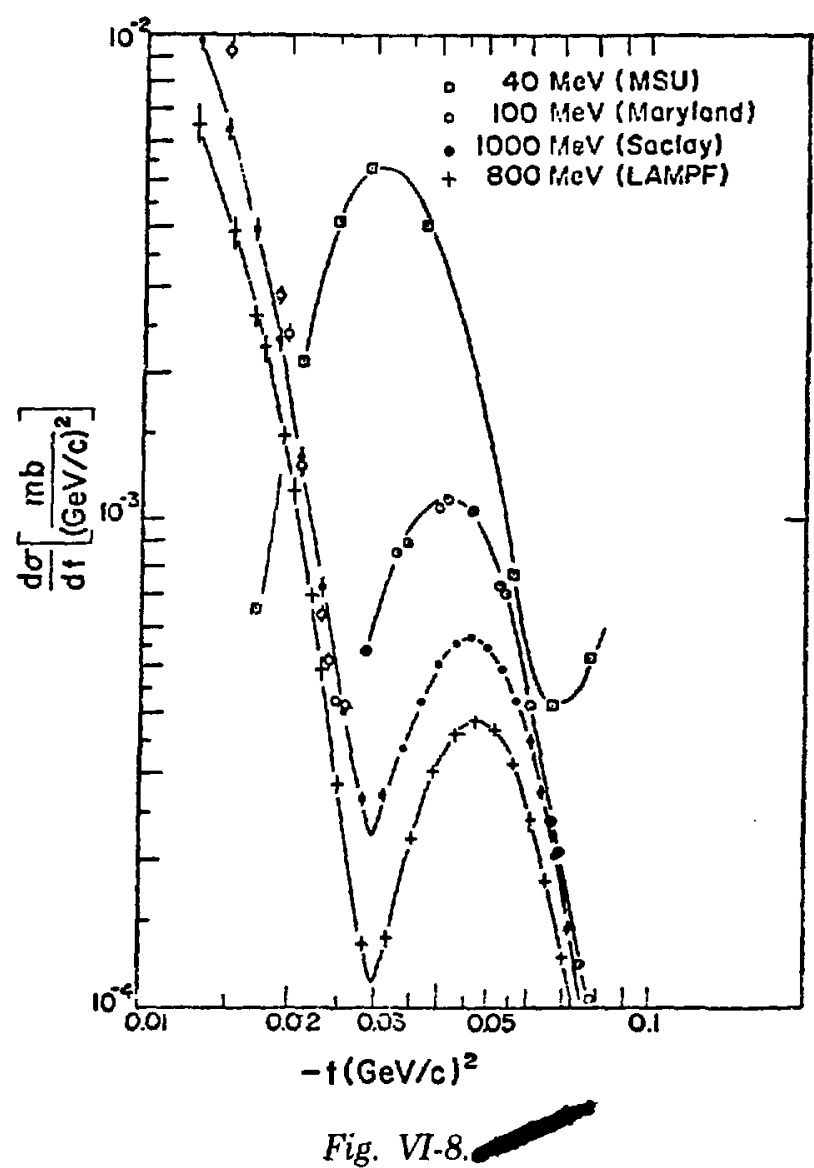

Invariant cross sections for proton scattering on ${ }^{58} \mathrm{Ni}$ for protons between $40-1000 \mathrm{MeV}$. The minima for the 800- and 1000-MeV experiments agree to better than $\pm 0.02 \mathrm{~F}$.

tical model calculation employing parameters from recent muonic $\mathrm{x}$-ray data.

The data and the results of the fits to $\Delta \sigma(\Omega)$ are presented in Fig. VT-9. These fits involve no free parameters, and the strongly correlated nature of the data should be kept in mind when evaluating the goodness of fit. Nete that for $\Delta \sigma(\Omega)$, the usual multiple-scattering currection does not appear since it is independent of nuclear alignment and cancels when the subtraction is performed. For $\Omega>0.2 \mathrm{sr}$, the fits are reasonably good. In this region, $\sigma(\Omega)$ is dominated by nonelastic processes since the elastic scattering is strongly forward peaked.

The fits to the $\Delta \sigma(\Omega)$ data are very good at $165 \mathrm{MeV}$, near the peak of the $(3,3)$ resonance, where the data indicate a small but statistically significant effect of $\sim 50 \mathrm{mb}$. At the other two energies, the data tend to be systematically less 


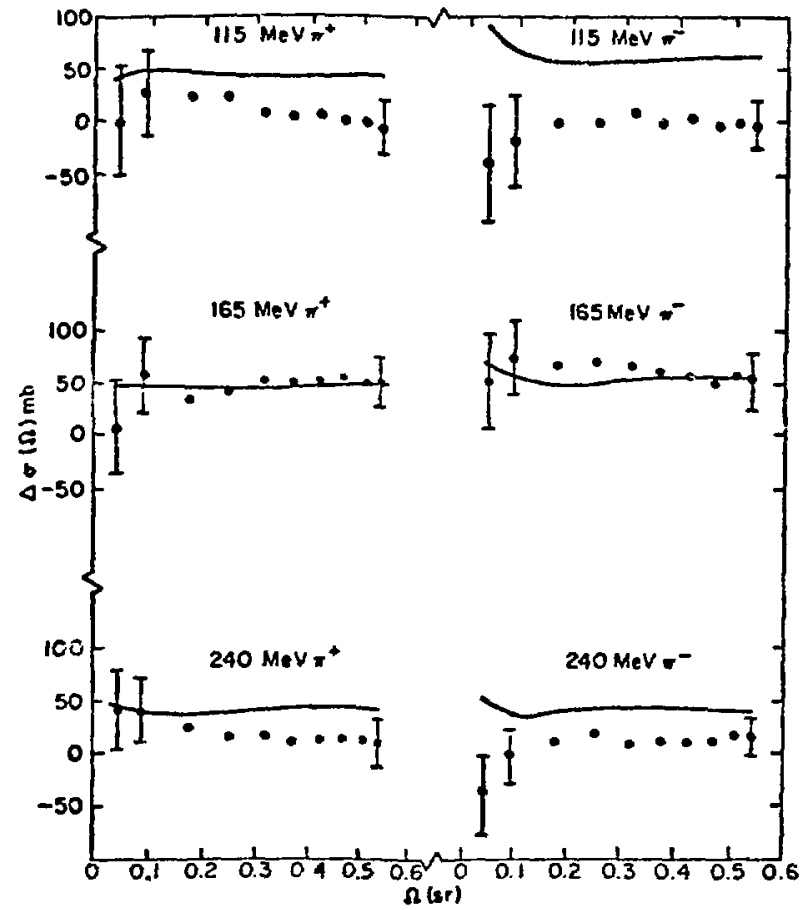

Fig. VI-9.

Experimental data on $\Delta \sigma(\Omega)$, the difference between the aligned and unaligned values of $\sigma(\Omega)$. The theoretical curves were obtained with the coupled-channels calculation.

than the theoretical prediction and are consistent with zero, although the theoretical curves are similar to those at $165 \mathrm{MeV}$. Because of statistical uncertainties in the data, however, it is impossible to determine whether the calculation fails at energies off the $(3,3)$ resonance or whether the entire body of data should be treated as consistent and averaged. The second interpretation lesdin, for example, to a value of $\Delta \sigma(\Omega)=22 \pm 10 \mathrm{lub}$ at $\Omega=$ $0.5 \mathrm{sr}$, which differs significantly from the theoretical prediction of $48 \mathrm{mb}$, and could imply that some modification is needed in the description of the deformed nuclear matter distribution.

In principle, it should be possible to apply Coulomb corrections to the data and extrapolate to zero solid angle to obtain estimates of $\Delta \sigma$. This was the first approach actually tried, and it led in most cases to small or negative values of $\Delta \sigma$ because of the downward trend of the first two points in five out of the six cases. This downward trend is not presently understood and is not reflected in any of the calculated curves. We suspect that it may not indicate a real cross-section trend, however, because it would imply that the alignment effect on the forward elastic cross section is opposite in sign from the geometrical effect. For a nucleus which must bear strong resemblance to a totally absorbing black spheroid, this would be difficult to explain. We have therefore preferred to present the entire body of data on $\Delta \sigma(\Omega)$ together with the theoretical curves.

Finally, we may ask how sensitive the present $\mathrm{ex}$. periment is to an angular variation in the dif. fuseness of the nuclear surface or to a difference in the neutron and proton deformation parameters. A variation in surface diffuseness can be introduced by allowing the diffuseness parameter $\mathrm{a}(\theta)$, in a Fermi-mass distribution, to vary with angle according $t o$ the prescription

$$
\mathrm{a}(\theta)=\mathrm{a}_{o}\left[1+\gamma \mathrm{Y}_{2}(\theta)\right]
$$

Figure VI-10 shows the effect of $\gamma$ on the calculated curve of $\Delta \sigma(\Omega)$ for $165-\mathrm{MeV} \pi^{+}$. Since the pions are strongly absorbed in the tail of the nuclear potential, negative values of $\gamma$, which make the nucleus more spherical in the tail region, decrease $\Delta \sigma(\Omega)$. A value of $\gamma=-0.36$ has been proposed for ${ }^{165} \mathrm{Ho}$ on the basis of muonic $x$-ray data, but a factor of $5 \mathrm{im}$ provement in the statistical precision of the present data would be required to distinguish this case from the one for $\gamma=0$.

Since positive pions interact more strongly with protons and negative pions with neutrons in the $(3,3)$ res mance region, the difference between $\Delta \sigma(\Omega)$ for positive and negative pions is sensitive to the neutron deformstion, assuming the proton deformation is known from Coulomb excitation ${ }^{8}$ and muonic $\mathrm{x}$-ray results. ${ }^{7}$ Figure VI-11 shows a calculation of this difference for $\beta_{n}=0.33$ and $\beta_{n}=0$ at $165 \mathrm{MeV}$. The present data are certainly consistent with $\beta_{n}=$ 0.33 , but here again much better statistical precision would be required to obtain useful new information on nuclear structure. 


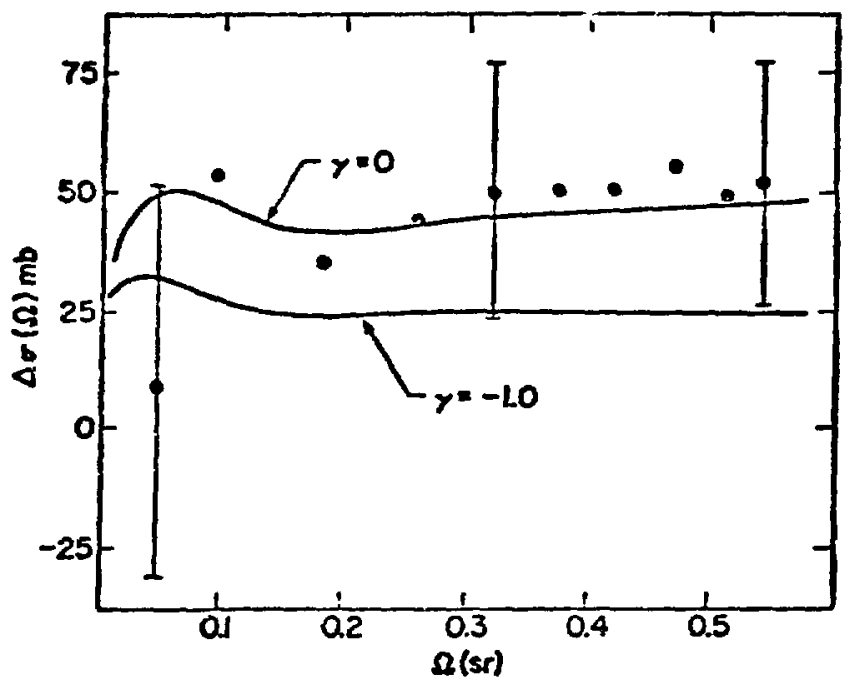

Fig. VI-10.

Calculated curves of $\Delta \sigma(\Omega)$ for $165-\mathrm{MeV} \pi^{+}$illustrating the effect of variation in the surface diffuseness parameter $\gamma$.

\section{The $\pi^{+} \mathrm{d} \rightarrow 2 \mathrm{p}$ Reaction at 100-500 $\mathrm{MeV}$}

(Exp. 201)

(Univ. of Virginia)

We are analyzing our data on the $\pi^{+} d \rightarrow 2 p$ reaction. The computer programs for analysis are still under development, but we have proceeded to the point where it is clear that the reaction is very cleanly separated from background. It also has been determined that the analysis can rely almost entirely on the TOF measurements for the two protons. We will use the trajectory measurements separately to obtain the centroids of the proton distributions in each arm, to guarantee that the solid angle is determined entirely by only one arm. The resulting simplicity in the analysis is especially welcome since we have about 750000 events to analyze. No cross sections are yet available.

\section{A Survey of Fion Single-Charge-Exchange Scattering Using Back-Angle Gamma Spec- troscopy (Exp. 303)}

(ANL, Northwestern Univ., LASL)

We recently began a series of measurements in which the $\pi^{0}$ energy spectrum and angular distribution are inferred from the energy spectrum and

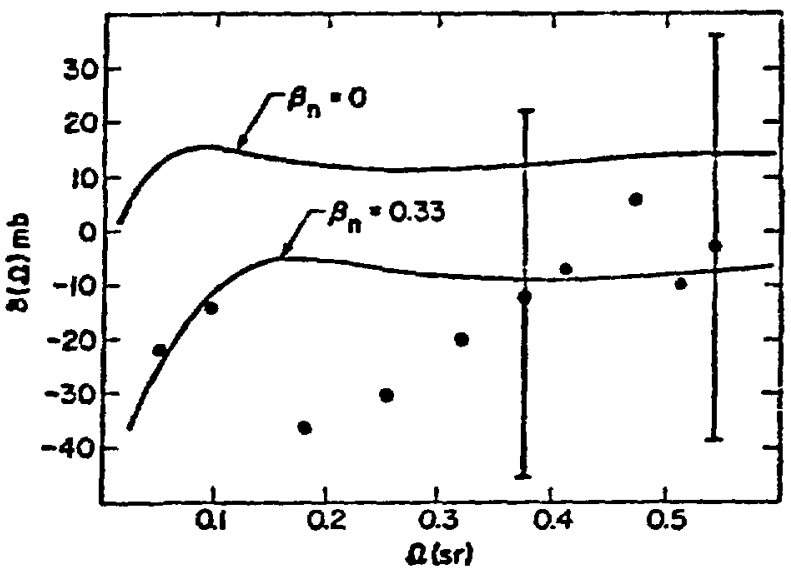

Fig. VI-11.

Culculated curves of $\Delta \sigma(\Omega)$, the difference betu'een $\Delta \sigma(\Omega)$ for $\pi^{+}$and $\pi^{-}$pions, at $165 \mathrm{MeV}$, illustrating the effect of varying the neutron deformation parameter $\beta_{2_{\bar{n}}}$ while fixing the proton deformation parameter at 0.33 .

angular distribution of the $\gamma$ rays emitted at back angles in the $\pi^{0}$ decay. The first results of a survey of the qualitative features of single-charge exchange using this technique are described here. A strong backward peaking of the energy-integrated angular distribution of the $\pi^{0}$ is observed for all nuclei.

The measurements were carried out on the LEP channel using pion fluxes of $\sim 2 \times 10^{7}$ pions/s with an incident energy of $100 \mathrm{MeV}$. The experimental configuration shown in Fig. VI-12 consisted of two cylindrical photon detectors viewing a target which lay on their common axis of symmetry. Two such detector pairs were used in these measurements. The essential elements of the Back-Angle Gamma Technique as used here were: 1) coincident observation of decay photons emitted with opening angles near $180^{\circ}, 2$ ) observation of the high-energy photon with a crude lead-glass spectrometer, and 3) observation of the low-energy photon with a large volume NaI(TI) spectrometer. The spectroscopy of the decay photon emitted near $180^{\circ}$ was possible because of the large Doppler shift of decay photons emitted by $\pi^{0} \mathrm{~s}$ with kinetic energies greater than $\approx 50 \mathrm{MeV}$. The NaI(TI) crystals were $25 \mathrm{~cm}$ in diameter, $30-\mathrm{cm}$ thick, and were surrounded by a plastic anticoincidence shield. For each coincident event, the energies of both of the detected $\gamma$ rays 


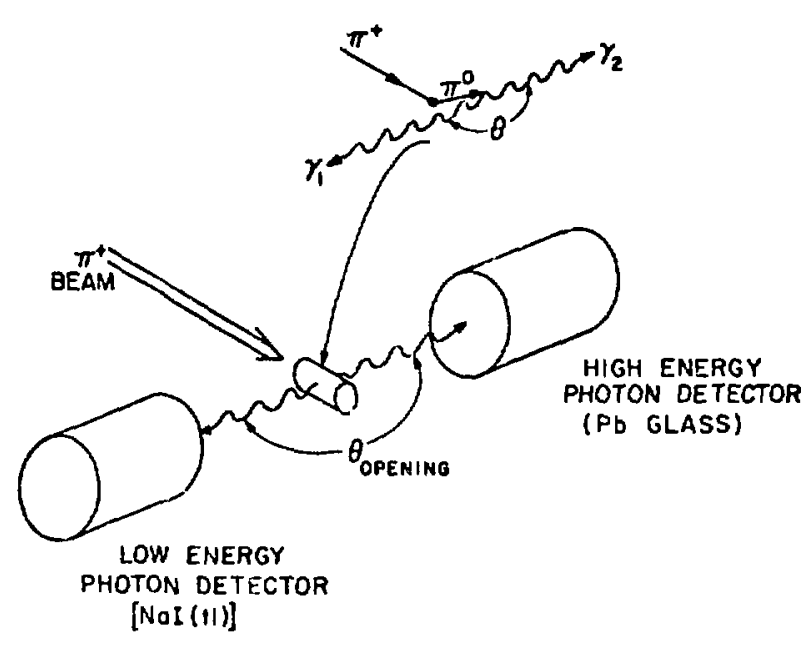

Fig. VI-12.

Experimental configuration showing a single$\pi^{0}$ detector pair. The inset shows a typical event and the corresponding photon opening angle, $\theta$.

were stored in the ANL PDP-11/45-based dataacquisition system. The kinematic regions corresponding to $\pi^{0}$ events of the $E_{\gamma}(\mathrm{NaI})$ vs $\Omega_{\gamma}$ (leadglass) spectrum were then identified and the $\pi^{0}$ spectra extracted.

The $\pi^{0}$ spectra observed with an ${ }^{16} \mathrm{O}$ target are shown in Fig. VI-13. Spectra were taken with $\pi^{+}$on $\mathrm{Be}, \mathrm{C}, \mathrm{O},{ }^{58} \mathrm{Ni}$, ${ }^{208} \mathrm{~Pb}$, and with $\pi^{-}$on $\mathrm{CH}_{2}$ and $\mathrm{C}$. In each case, laboratory angles of $40^{\circ}$ and $120^{\circ}$ were measured. For all targets the spectra had the same gross shape, roughly described as a peak about 60 $\mathrm{MeV}$ wide centered at about $60 \mathrm{MeV}$, although the average $\pi^{0}$ energy was slightly greater at $40^{\circ}$. The energy resolution and efficiency calibration of the experiment was established by a $\mathrm{CH}_{2}-\mathrm{C}$ difference measurement of the $\pi^{-} p \rightarrow \pi^{0} n$ reaction using cross sections calculated from known $\pi$-nucleon phase shifts. Figure VI-14 shows energy ${ }^{\prime}$-integrated $\pi^{0}$ cross sections per target neutron al the two angles measured for each of the ta. rets studied. The cross section per neutron varies $a_{5} A^{-0.4}$ at both angles. The $\pi^{0}$ yield is backward peaked with the $\mathrm{d} \sigma\left(40^{\circ}\right) / \mathrm{d} \Omega: \mathrm{d} \sigma\left(120^{\circ}\right) / \mathrm{d} \Omega$ ratio virtually constant at 0.3 , near the value of the free-nucleon reaction $\pi^{-}+$ $\mathrm{p} \rightarrow \mathrm{n}+\pi^{\circ}$.

If the nucleus were nearly transparent to pions, the characteristic backward peaking of the yield

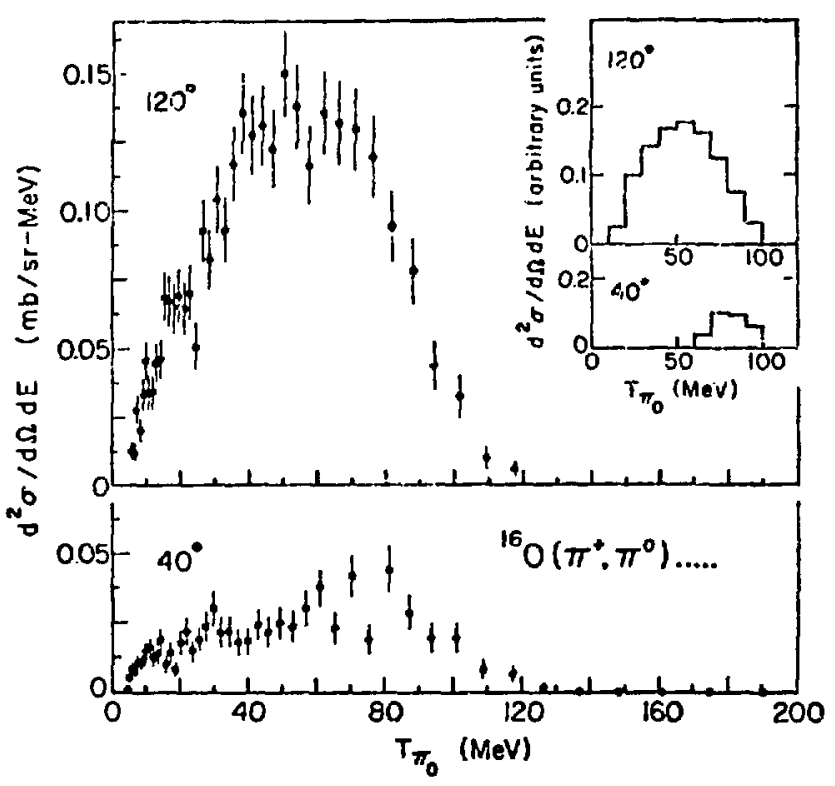

Fig. VI-13.

Differential cross section for single-charge $\mathrm{ex}$ change by ${ }^{10} \mathrm{O}$ at $40^{\circ}$ and $120^{\circ}$. The inset shows the cross section shapes calculated for chargeexchange scattering by a Fermi gas.

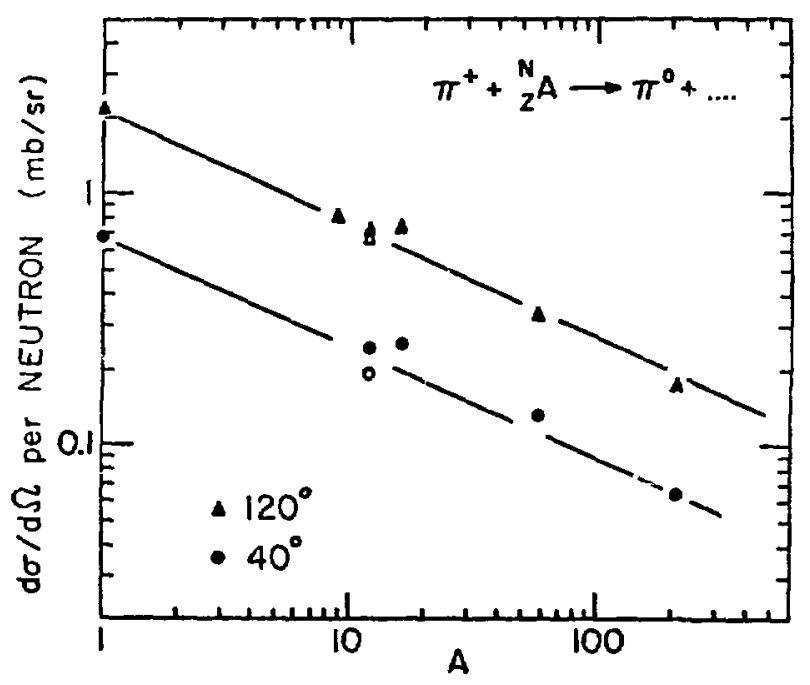

Fig. VI-14.

Differential cross section for single-charge exchange as a function of atomic mass. The points for $A=1$ are based on the assumed equality of $\pi^{-}+p \rightarrow \pi^{0}+n$ and $\pi^{+}+n \rightarrow \pi^{0}+$ $p$. The open points for $A=12$ were taken with negative pions. 
would be an indication that the asymmetry of the $\pi$ nucleon process persists in $\pi$-nucleus charge exchange through a quasi-flee type of scattering. In this case, the charge-exchange cross section per neutron would be nearly independent of mass. Instead, the trend of rapid decrease in cross section per neutron with increasing mass, evident in our data (Fig. VI-13) indicates strong absorption of the pion in the nuclear interior. The $\pi^{0}$ energy spectrum at $40^{\circ}$ is in itself evidence that the constant value of the ratio of $40^{\circ}$ - to $-120^{\circ}$ yield is not simply a reflection of the free-nucleon cross section. The insets to Fig. VI-13 show results of a simple calculation of charge-exchange scattering by a proton-neutron Fermi gas. The energy and angular dependence of the differential cross sections were taken from $\pi$ nucleon phase shifts and the effects of Pauli blocking were included in the computation. The calculated curve at $120^{\circ}$ reproduces rather well the shape observed experimentally. However, the distribution calculated for $40^{\circ}$ is much sharper than the data with a higher mean energy than is observed. As the calculation indicates, some spreading of the $\pi^{0}$ energy can be expected, but no $\pi^{0}$ with less than $\approx 60 \mathrm{MeV}$ should be observed at $40^{\circ}$ if the maxin um Fermi energy is of the order of $40 \mathrm{MeV}$. The low-energy $\pi^{0} \mathrm{~s}$ that are observed at the forward angle are most likely the result of processes more crmplex than quasi-free charge exchange on a single nucleon. The quasi-free component would be expected to have an even stronger angular asymmetry than the free-nucleon reaction because of Pauli blocking of states in the forward scattering. The Fermi gas calculation gives an enhancement of $\mathrm{d} \sigma\left(120^{\circ}\right) / \mathrm{d} \Omega$ relative to $\mathrm{d} \sigma\left(40^{\circ}\right) / \mathrm{d} \Omega$ of 7.1 at $100 \mathrm{MeV}$. Thus, it is evident that quasi-free scattering is not important at forward angles.

\section{Theory}

\section{$\mu^{-}$Capture Ratios}

Theoretical calculations based on the Fuzzy Fermi-Teller Model ${ }^{9}$ have been carried out to compare with experimental results for $\mu^{-}$capture in three series of solid binary systems: 1) alkali halides, 2) metal oxides, and 3) metal solutions. Modified Thomas-Fermi atomic potentials were used that accommodate fractional ionization. In addition, atomic dipole polarizabilities were included, together with energy gaps (for insulators) and conduction bandwidths (for conductors). Much of the experimental data used in the comparisons were obtained in Exps. 60 and 288.

The theoretical capture ratios were found to be sensitive to the radius ratios used for the binary systems. Since the effective radius ratio is a measure of the ionicity of a chemical bond, this technique may provide new information on ionicities - a subject of recent controversy. Results for the alkali halides are presented in Fig. VI-15. Theoretical results using Pauling's ${ }^{10}$ ionicities and Phillip's ${ }^{11}$ ionicities are connected by smooth

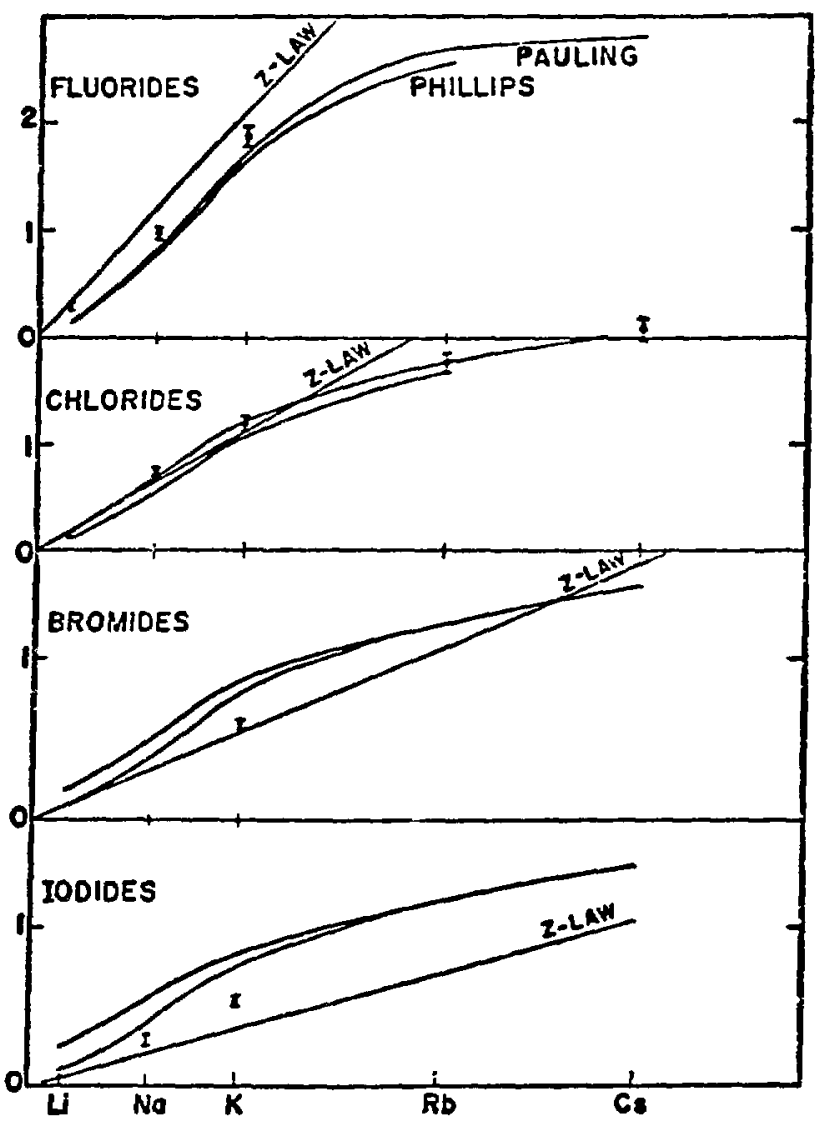

Fig. VI-15.

Negative-muon capture ratios for alkali-halide solids. Theoretical results, using Pauling's ionicities (upper curve) and Phillips' ionicities (lower curve), as well as the Fermi-Teller ZLaw, are shown as smooth curves. Experimental points $\Phi$ indicate data from Exp. 60. 
TABLE VI-V

NEGATIVE-MUON CAPTURE RATIOS FOR METAL OXIDES

\begin{tabular}{|c|c|c|c|c|c|}
\hline \multirow[b]{2}{*}{ Oxide } & \multirow[b]{2}{*}{$\begin{array}{c}\text { Experimental } \\
\text { Ratios }\end{array}$} & \multicolumn{4}{|c|}{ Theoretical Ratios } \\
\hline & & $\begin{array}{l}\text { Deduced } \\
\text { Ionicities }\end{array}$ & $\begin{array}{c}\text { Pauling } \\
\text { Ionicities (i) }\end{array}$ & $\begin{array}{c}\text { Phillips } \\
\text { Ionicities (i) }\end{array}$ & Z-Law \\
\hline $\mathrm{BeO}$ & $0.12 \pm 0.04$ & $(0.65-0.95)$ & $0.18(0.632)$ & $0.20(0.601)$ & 0.50 \\
\hline $\mathrm{MgO}$ & $0.83 \pm 0.07$ & $(0.66-0.75)$ & $0.79(0.734)$ & $0.65(0.841)$ & 1.50 \\
\hline $\mathrm{CaO}$ & $1.45 \pm 0.09^{\mathrm{a}}$ & $(0.78-0.87)$ & $1.53(0.790)$ & $1.26(0.913)$ & 2.50 \\
\hline $\mathrm{ZnO}$ & $2.66 \pm 0.32$ & $(0.38-0.58)$ & $2.26(0.594)$ & $2.18(0.619)$ & 3.75 \\
\hline $\mathrm{SrO}$ & & & $2.46(0.790)$ & $1.98(0.926)$ & 4.75 \\
\hline $\mathrm{CdO}$ & $2.47 \pm 0.22^{\mathrm{a}}$ & $(0.69-0.84)$ & $3.14(0.555)$ & $2.94(0.613)$ & 6.00 \\
\hline $\mathrm{BaO}$ & $2.27 \pm 0.22$ & $(0.86-1.0)$ & $2.61(0.815)$ & & 7.00 \\
\hline
\end{tabular}

curves. The experimental data are represented by bars, with LAMPF data indicated. The FermiTeller Z-Law predictions are included as a basis for comparison. Within the context of this model, the data do not unequivocally favor one set of ionicities over the other. Assuming the two sets of ionicities represent a reasonable range, the model does surprisingly well, especially considering its semiclassical and statistical nature. Significant deviations from the Z-Law are pr licted, pointing to a need for more data, especially ine rubidium and cesium compounds.

Results for metal oxides are presented in Table VI-V. Here we show the predicted capture ratios us- ing both sets of ionicities as well as the ionicity range concluded from the data based on our model, Again, the agreement is surprisingly good. Further data on medium-ionic compounds, such as sulfides of these metals, would also provide interesting comparisons.

Results from metal solutions are presented in Table VI-VI. Relative concentrations of each metal are given in parenthesis. Excellent agreement between theory and experiment is obtained for nickelcopper, zinc-silver, and silver-gold, while for magnesium-cadmium and aluminum-copper the agreement is rather poor. This discrepancy is not understood at present but is being pursued further.

TABLE VI-VI

NEGATIVE-MUON CAPTURE RATIOS FOK METAL SOLUTIONS

\begin{tabular}{|c|c|c|c|}
\hline Solution (Rel. Conc.) & Experiment & Theory & Z-Law \\
\hline $\mathrm{Mg}(0.15)-\mathrm{Cd}(0.85)$ & $0.318 \pm 0.025$ & 0.741 & 0.250 \\
\hline $\mathrm{Al}(0.15)-\mathrm{Cu}(0.85)$ & $0.278 \pm 0.014^{\mathrm{a}}$ & 0.785 & 0.448 \\
\hline $\mathrm{V} \quad(0.50)-\mathrm{Nb}(0.50)$ & $0.935 \pm 0.044$ & 0.723 & 0.561 \\
\hline $\mathrm{Ni}(0.48)-\mathrm{Cu}(0.52)$ & $0.926 \pm 0.043$ & 0.970 & 0.966 \\
\hline $\mathrm{Zn}(0.15)-\mathrm{Ag}(0.85)$ & $0.910 \pm 0.046^{\mathrm{a}}$ & 0.890 & 0.638 \\
\hline $\mathrm{Ab}(0.40)-\mathrm{Au}(0.60)$ & $0.787 \pm 0.074$ & 0.740 & 0.595 \\
\hline
\end{tabular}

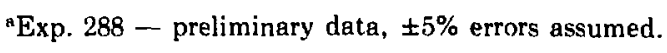




\section{Programming for Experiments}

A new version of the off-line analysis program was developed for Exp. $328(\mu \rightarrow$ e $\gamma)$. A "stage-zero" program was written to combine the reading of raw data tapes and the processing of best tracks using $\chi^{2}$ minimization. Thus the writing and handling of large intermediate files was eliminated. Tape reading speed was improved with a new KS90 utility for use with a TU10 tape drive. All computed quantities for events which pass specified tests are written on disk for unlimited histogramming by a special plotting program. Events which pass all tests are written on a disk file for later transfer to tape and final processing on the CDC6600. The stage-zero program was adapted for the 6600 and will be modified for the final stage of analysis.

A sy,$Q$ data-acquisition program was developed for Exp. 96 (Low-Energy T-p Elastic Scattering) by starting from the $Q$ acquisition program used by Exp. 328. Some of the features taken over include: an event-manager task which distributes event dita from the analyzer to other tasks; a task to list event data upon request; a simplified loading procedure used after a reboot to install Q tasks; and the plotting package, PLT, which allows the operator to begin, end, change, and display sets of histograms and live dot plots. New features for Exp. 96 include PLT capabilities for generating and displaying two-dimensional stored plots, and a new PIC, the schematic event diagram on the 4010 which shows chamber hits and scintillator pulse heights.

\section{REFERENCES}

1. "Medium-Energy Physics Program, November 1, 1976 - January 31, 1977," Los Alamos Scientific Laboratory report LA-6819-PR, pp. 86-87 (June 1977).
2. N. T. Porile, B. J. Dropesky, and R. A. Williams, Phys. Lett. 67B, 43 (1977).

3. B. W. Allardyce, C. J. Batty, D. J. Baugh, E. Friedman, G. Heymann, M. E. Cage, G. J. Pyle, G.T.A. Squier, A. S. Clough, D. F. Jackson, S. Murugesu, and V. Rajaratnam, Nucl. Phys. A209, 1 (1973).

4. M. K. Jakobson, G. R. Burleson, J. R. Calarco, M. D. Cooper, D. C. Hagerman, I. Halpern, R. H. Jeppesen, K. F. Johnson, L. D. Knutson, R. E. Marrs, H. O. Meyer, and R. P. Redwine, Phys. Rev. Lett. 38, 1201 (1977).

5. M. Jacob and O. Kofoed-Hansen, Nucl. Phys. B17, 565 (1970).

6. H. VanKempen, A. R. Midema, and W. J. Huiskamp, Physica 30, 229 (1964); G. Brunhart, H. Postma, and V. L. Sailor, Phys. Rev. 137, B1484 (1965); and M. Krusius, A. C. Anderson, and B. Holmstrom, Phys. Rev. 177, 910 (1969).

7. R. J. Powers, F. Boehm, P. Vogel, A. Zehnder, T. King, A. R. Kunselman, P. Roberson, P. Martin, G. H. Milier, R. E. Welsh, and D. A. Jenkins, Phys. Rev. Lett. 34, 492 (1975).

8. B. Elbek, "Determination of Nuclear Transition Probabilities by Coulomb Excitation" (Thesis, Ejnar Munksgaard Forlag, Pub., Copenliagen, Denmark, 1963).

9. M. Leon and J. H. Miller, Nucl. Phys. A282, 461 (1977).

10. L. Pauling, The Nature of the Chemical Bond (?rd ed., Cornell Univ. Press, 1960).

11. J. C. Phillips and J. A. VanVechten, Phys. Rev. Lett. 22, 705 (1969). 


\section{NUCLEAR CHEMISTRY}

\section{Nuclear Chemistry Laboratory}

\section{Data-Acquisition System (DAS)}

The floating point processor for the PDP-11/34 has been received and installed. FORTRAN IV PLUS (F4P) is now being added to the operating system. Among other system additions, a new version of the Versatec plotting package was implemented to give a fourfold increase in operating speed.

The IBM 026 keypunch has been connected to the CAMAC system for punched card output, and already has seen heavy use, principally for Exp. 111 .

Automatic recording of decay data has been used primarily for Exp. 67. The DAS has continued to be heavily used by Exps. 111, 142, and 292 for off-line analysis of multichannel $\gamma$-ray spectral data.

\section{Counting Room Equipment}

Faster and more reliable electronic modules were installed as replacements on our second $\gamma-\gamma$ coincidence (annihilation radiation) scintillation counter and singles $\gamma$-ray scintillation counter. All indications are that substantial improvements in these systems have been achieved.

A modest step was taken to start replacing obsolete electronic components for our $\alpha$ and $\beta$ proportional counters. Two sets of preamplifiers, amplifiers, hv supplies, and scaler-timers for the gasflow $\beta$ proportional counters were obtained and soon will be checked out.

\section{Pneumatic "Rabbit" System}

Considerable effort continues to be expended in completing the development of this system. A gravity-operated, carrier-sensing electrical switch has been designed and fabricated for the end of the "snake" at the Energetic Neutron Station at the A-6 beam stop. While benchtop testing of the new springless switch indicates that it functions satisfactorily, we have no operational experience yet with its performance in the very hostile environment adjacent to the beam stop (flux of $>10^{11} \mathrm{n} / \mathrm{cm}^{2} / \mathrm{s}$ of $E_{n}$ $>20 \mathrm{MeV}$ ).

Two Control Console timers for timing the duration of target exposures at the Neutron Station and Proton Station (Line B) were wired into the system and checked out. Automatic express transaction wiring logic was corrected and tested. These express transactions are now available to the experimenter: Hot Cell to Neutron Station to Hot Cell; Chem Hood B (near Hot Cell) to Hot Cell to Neutron Station; Neutron Station to Hot Cell to Chem Hood B; Hot Cell to Proton Station to Hot Cell; Nuclear Chemistry Laboratory to Hot Cell to Neutron Station; and Neutron Station to Hot Cell to Nuclear Chemistry Laboratory.

Schematic drawings of the complete control circuitry for the entire rabbit system have been made. Also, a wiring schematic for the snake drive mechanism and the digital target position indicator for the Neutron Station were drawn up. A torquelimiter clutch assembly was installed on the snake drive mechanism to protect the snake in the event of excessive resistance to insertion.

The two 4-position diverters used to control the traffic pattern of the target carriers passing through Switch Pit No. 1 had to be modified with a chainurive tightener in order to insure good alignment of the air tubes.

The redesign of the remote targeting station in Line $B$ required shifting the pivot axis of he bellows. A new bellows has been ordered and the necessary flanged spool extension and elongated trunnion brackets have been fabricated. Present plans now call for modifications to the "trombone" and completion of the redesigned targeting station during the December-January shutdown of LAMPF.

Heavy-duty terminals required to replace the initial lightweight and unreliable terminals in the Hot Cell, Chem Hood B (2), and at the Nuclear Chemistry Laboratory have been designed and fabricated. Completion and installation of these terminals is expected to be carried out during the next quarter. 


\section{WNR Rabbit System}

Work on development of the 38-mm rabbit system at the WNR facility has continued. The J-tube integrity testing has been completed. The mechanism for J-tube telescoping has been fabricated and checked out, but not installed. Shipment of the blower package to be installed in the experimental trailer has been made by the manufacturer. All components for control of the system have been fabricated. The rabbit receiving station and diverter have been received. Work on refurbishing the experimental trailer is proceeding, including fabrication of the glove box to house the receiving station.

\section{Research Activities}

At the Thin Target Facility, Exp. 86 has received severa! hundred hours of $800-\mathrm{MeV}$ proton beam time on a platinum target in order to determine yields of light neutron-rich fragments relative to yields from uranium targets used in the past. Experiment 106 received two target irradiations with $800-\mathrm{MeV}$ protons in Line $\mathrm{B}$; one was to determine the yields of and radiochemical separation procedures for the spallation products from molybdenum. The participants on Exp. 243 carried out a long irradiation of a thin uranium target in the scattering chamber in the Nuclear Chemistry Cave on Line $B$ in order to determine the differential ranges of scandium and barium nuclides from the interaction of ${ }^{238} \mathrm{U}$ with $800-\mathrm{MeV}$ protons. Many dozens of short irradiations were carried out at the Energetic Neutron Station of the Rabbit System by the Exp. 111 team in their studies of the decay properties of 12 - $\min { }^{44} \mathrm{Ar}, 21-\mathrm{s}{ }^{45} \mathrm{Ar}$, and $\sim 9-\mathrm{s}{ }^{46} \mathrm{Ar}$ produced by $(n, 2 p 3 n),(n, 2 p 2 n)$, and (n,2pn) reactions, respectively, on ${ }^{48} \mathrm{Ca}$.

\section{Theoretical Support}

\section{The VEGAS Intranuclear Cascade Program}

The Third VEGAS Review meeting was held on August 26, 1977 at LAMPF. in attendance were D. Cochran (MP-DO), B. J. Dropesky (CNC-11), D. G. Foster (T-2), G. Friedlander (BNL), J. N. Ginocchio (T-5), M. Hillman (BNL), V. G. Lind (Utah State
University), N. Metropolis (T-7), H. Plendl (Florida State University), G. Stephenson (T-5), and A. L. Turkevich (University of Chicago and LASL). Many details of the intranuclear cascade model (INC) were discussed. Covered were the questions of the correct way to take into account the refraction of nucleons as they move through the nuclear medium, and the depletion of the nuclear density as particles are struck. The adequacy of using an evaporation model for medium-energy reactions which does not include angular momentum effects was questioned, and it was suggested that a new evaporation program, JULIAN, which takes angular momentum effects into account, be used with the VEGAS intranuclear cascade program. It was also suggested that an effort be made to understand the results of the INC using simple models of nuclear reactions.

Several VEGAS calculations have been performed recently relevant to a number of experiments at LAMPF. In one of these experiments, ${ }^{1}$ the cross sections for the radioisotopes of arsenic produced from two different pion-induced paths are measured. One is ${ }^{78} \mathrm{Ge}\left(\pi^{+}, \mathrm{xn}\right)^{78-x} \mathrm{As}$, and the other is ${ }^{76} \mathrm{Se}\left(\pi^{-}, \mathrm{xn}\right)^{78-x} \mathrm{As}$, both with $180-\mathrm{MeV}$ pions. In both cases the INC underestimates the cross section by 10 to $30 \%$ for $\pi^{+}$and from 30 to $60 \%$ for $\pi^{-}$. This is consistent with other experimental results for which the discrepancy is larger with $\pi^{-}$beams than with $\pi^{+}$beams. Another experiment is the observation of the $\pi^{0}$ spectra at different angles.2 The fact that $\pi^{0}$ production is backward peaked is reproduced by the calculation. Furthermore, the shape of the spectrum at backward angles $\left(\theta=120^{\circ}\right)$ is reproduced fairly well. However, at forward angles $\left(\theta=40^{\circ}\right)$, the calculated spectrum is peaked more towards higher energy $\pi^{0} \mathrm{~s}$ than the preliminary experimental results indicate. Another relevant experiment is the extensive measurements of products produced in the nickel isotopes by nieasuring in-beam gamma rays. ${ }^{3}$ The preliminary results indicate that the experiment produces more cross section for products with 5-6 nucleons removed from the target than is calculated by the intranuclear cascades. These and other experiments that show discrepancies with the intranuclear cascades indicate that there are serious gaps in our understanding of pion reactions.

One of the discrepancies is the fact that the total pion reaction cross section calculated by the INC for ${ }^{12} \mathrm{C}$ through the resonance region is $\sim 20 \%$ lower than 
that measured. ${ }^{4}$ Since the pion is interacting strongly with the nucleus in the resonance region, it is possible that the deformation of ${ }^{12} \mathrm{C}$ may have an effect on this cross section. This possibility is being investigated analytically using transport theory and will be reported on at the Annual Meeting of the American Physical Society in San Francisco in January 1978.

\section{REFERENCES}

1. L. B. Church (Spokesman), "A Comparison of $\left(\pi^{+}, \mathrm{xn}\right)$ and $\left(\pi^{-}, \mathrm{xn}\right)$ Reactions," LAMPF Exp. 320 .
2. H. E. Jackson (Spokesman), "A Survey of Pion Angle Charge Exchange Scattering Using Back Angle Gamma Spectroscopy." LAMPF Exp. 303.

3. R. E. Segal (Spokesman), "Interactions of Stopped Negative Pions with Complex Nuclei," LAMPF Exp. 121.

4. F. B. Binon, ?. Duteil, J. P. Garron, J. Gorres, L. Hugon, J. P. Peigneux, C. Schmit, M. Spighel, and. J. P. Stroot, Nuclear Physics B17, 168 (1970). 


\section{PRACTICAL APPLICATIONS OF LAMPF}

(Summarizes work being performed under the auspices of USDOE Division of Physical Research, LSDOE Division of Biomedical and Environmental Research, and the National Cancer Institute.)

\section{Pion Biomedical Program}

\section{Channel Control System Development}

Two treatment couches were installed, one in the treatment room and one in the simulator room. A PDP-11/03 microprocessor was purchased and interiaced to the treatment room couch. Extensive software was written to move the couch in four direcins (three linear and one rotstional), and to read the corresponding four components of position. Optical shaft encoders were installed on the couch to encode position data. A 16-bit multiplexer was designed and built so that up to eight encoders could be read independently by the microprocessor and the main computer. The software for moving and monitoring the couch represents the first phase of a completely new patient-treatment software package.

Another maajor accomplishment was the completion of a new channel control system software package. The single task for setting magnets, slits, etc., has been replaced by a separate task for each device. Each task is interrupt-driven from its respective hardware device, and hence can be in a suspended state while it waits for its device to reach the setpoint. This technique has greatly reduced the load on the main computer during channel setup.

Many improvements have been made to the hardware. The hydraulic-driven proton target mechanism has been replaced by a dc motor-driven mechanism. This target mechanism has been completely redesigned to eliminate many of the bugs in the old mechanism. A linear power amplifier was designed and built for the dc motor. The complicated gear-train/rotary-position pot has been replaced by a simple linear-pusition pot. An optical shaft encoder has been added so that the target position can be read more accurately by both the main computer and the microprocessor. A water-cooled graphite target has been designed to be put on the new target mechanism. A new wedge changer has been designed and built, as well as a new controller for it. The related software has iso been updated.

A newly designed range shifter has been installed that, because it is more compact, allows the physician/radiation therapist to better visualize the treatment area when the range shifter is in position. It can be operaied from the main computer or from the PDP-11/03 microprocessor. An optical shaft encoder was also added to the range shifter, and this, too, can be read by the main computer and the microprocessor simultaneously. A new monitoring panel was built for the range shifter so that all of the main operating characteristics can be monitored and recorded. An electronic package (servo controller) has been designed for the new range shifter. The new electronic package takes digital inputs from the computer or microprocessor rather than analog inputs, so now there is no problem with bias offsets from different inputs. A range-shifter function verification task has been written, so the computer knows whether or not the range shifter is behaving properly during treatment.

A dynamic collimator has been designed. This collimator will use a shaft encoder on each jaw to accurately determine the position of the jaw. Static collimators have been coded so the computer can read which collimator is being used with which patient. The pion beam cannot be turned on if the wrong collimator is in place.

\section{-Channel Tuning/Beam Development}

(Exp. 270)

(LASL)

The design of new beams has proceeded very rapidly. The original-design fan tune for the channel produces a beam without sufficient uniformity along the long dimension of the stopping region. Using ray-tracing methods and our improved TRANSPORT matrices, we traced the problem to beam loss in one of the output quadrupoles. A new fan tune was then calculated allowing for a smaller beam size in this magnet. The resulting measured beam is considerably more uniform. Other fan tunes have been calculated and subsequently measured. The position of the beam waist in the narrow dimension of 
the fan has been varied. On the first try, the waist position came to within $1 \mathrm{~cm}$ of the desired position.

A number of new static therapy beams have also been developed. Reams are available at several energies for $8 \mathrm{~cm}$ by $4-\mathrm{cm}$ treatment fiels.s. For exceptionally large fields, $\mathrm{cn} 8-\mathrm{cm}$ by $\mathrm{s}-\mathrm{cm}$ beam and a $15-\mathrm{cm}$ by $15-\mathrm{cm}$ beam are $y=d$ either singly or abutted. A low-momentum iung nodule tune was developed to have the sharpest possible lateral and distal dose falloff. Lowered pion momentum reduces multiple Coulomb scattering degradation of the edges and reduces the spread in pion range of the fixed-percentage momentum bite.

\section{- Therapy Beam Development - Microdosimetry (Exp. 272) (LASL)}

Broad-Beam Microdosimetry. Microdosimetry data have been obtained for one of the collimated broad beams. A depth-drse curve of this beam tune is shown in Fig. VIII-1. This beam, flat in physical dose throughout the stopping region, was chosen in order to eliminate a variable (absorbed dose) in the interpretation of the results. Data were taken in the upstream portion of the peak region, the center, the distal edge, and the downstream portion. These positions are indicated in Fig. VIII-1.

The spectra have been obtained using a Rossitype proportional counter with an equivalent

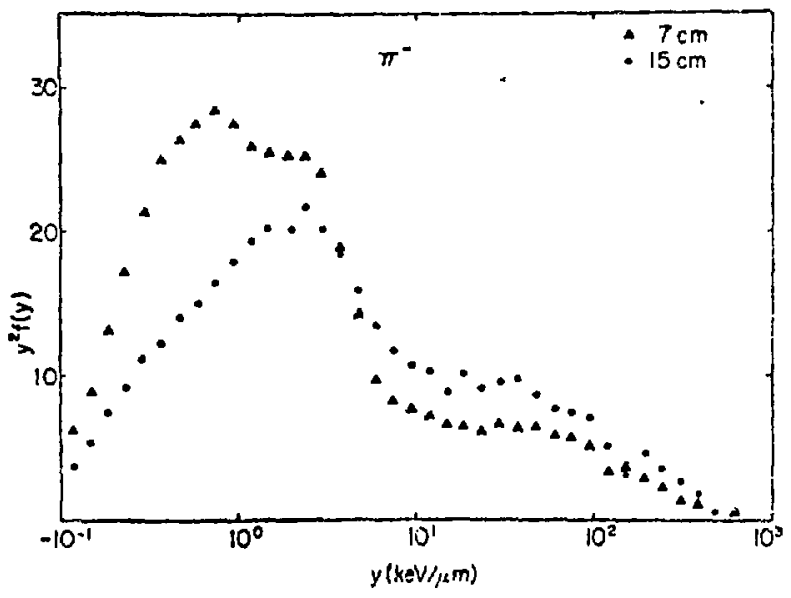

Fig. VIII-2.

Microdosimetry spectra at 7 and $15 \mathrm{~cm}$ (see also Fig. VIII-1).

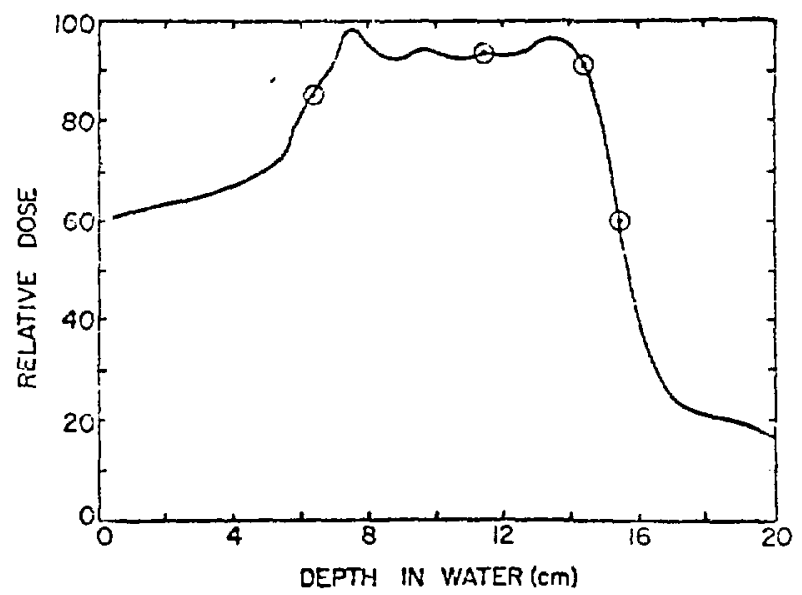

Fig. VIII-1.

Depth-dose curve for the $\delta$-cm beam. The positions where the microdosimetric spectra uere measured are indicated by circles.

diameter size of $2 \mu \mathrm{m}$. The counter was filled with propane-based tissue-equivalent gas. The measure ments were performed in a water phantom. The elliptical-shaped collimator had linear axial dimensions of 9 by $11 \mathrm{~cm}$.

The resulting spectra are displayed i Figs. VIII-2 and -3. The general structure of these spectra as weli as their possible break into contributions coming from various particle types has been discussed previously ir he case of a narrow beam.

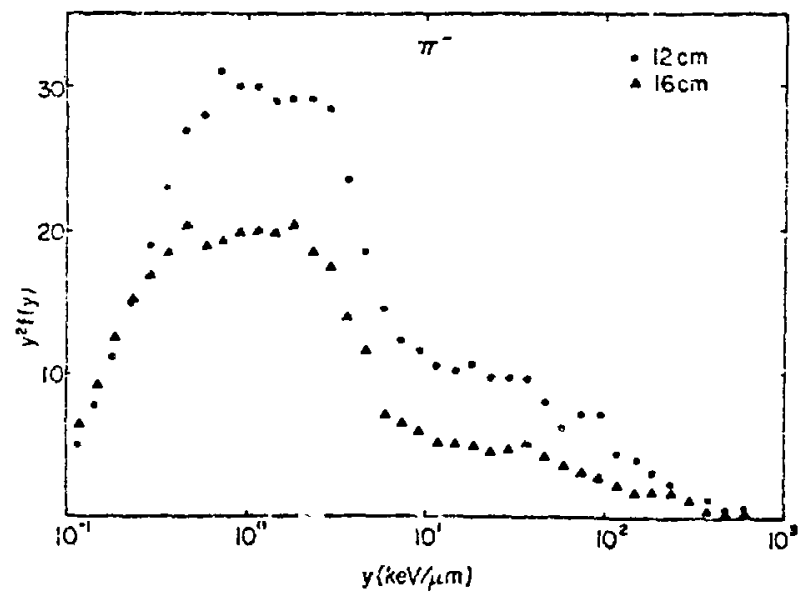

Fig. VIII-3.

Microdosimetry spectra at 12 and $16 \mathrm{~cm}$ (see also Fig. VIII-1). 
In Table VIII-I we present calculated values of the RBE and OER for these measurements. The dose mean lineal energies are listed also. The theoretical calculations used the Kellerer-Rossi model with a $y_{0}$ $=125 \mathrm{keV} / \mu \mathrm{m}$. The RBE and OER values correspond to a dose of $350 \mathrm{rad}$.

Figure VIII-4 shows the fraction of dose resulting from events having lineal energies above a given value vs the lineal energy. As can be seen from Fig. VIII-4, the high-LET component increases with increasing depth until the distal edge $(16 \mathrm{~cm})$. There we have at most $7 \%$ of the dose coming from events with lineal energy above $50 \mathrm{keV} / \mu \mathrm{m}$. Downstream from the peak, the high-LET component decreases. These results are compared with our ineasurements performed at Berkeley using a $557-\mathrm{MeV} / \mathrm{amu} \mathrm{Ne} \mathrm{N}^{10+}$ broad beam shown in Fig. VIII-5.

Neutron Microdosimetry. In these measurements we studied the contribution from star-produced neutrons and photons at the beam edges and beyond the volume of treatment. The data were taken under experimental conditions similar to those described above. The microdosimetry spectra obtained in the center of the peak, and in the same plane but $8 \mathrm{~cm}$ off axis, indicate that for this treatment volume $(\sim 1 \ell)$ the neutron dose at $\mathrm{y}=8 \mathrm{~cm}$ is $\sim 3 \%$ of the peak dose. The neutron dose in the treatment volume is estimated to be $\sim 15$ to $20 \%$ of the total dose. Calculations based on the present spectra show that the RBE should be between 1.1 and 1.4.

Neutron Biology. In order to further assess the biological effect of neutrons outside the treatment

\section{TABLE VIII-I}

\section{CALCULATED VALUES OF RBE-OER FOR V-79 CELLS AT 350-RAD OF PIONS}

\begin{tabular}{|c|c|c|c|}
\hline $\begin{array}{c}\text { Position } \\
\text { (cm) }\end{array}$ & $\begin{array}{c}y_{d} \\
(\mathrm{keV} / \mu \mathrm{m})\end{array}$ & RBE & OER \\
\hline 7 & 13.0 & 1.17 & 2.15 \\
\hline 12 & 13.9 & 1.19 & 2.19 \\
\hline 15 & 21.1 & 1.26 & 2.11 \\
\hline 16 & 10.5 & 1.14 & 2.19 \\
\hline
\end{tabular}

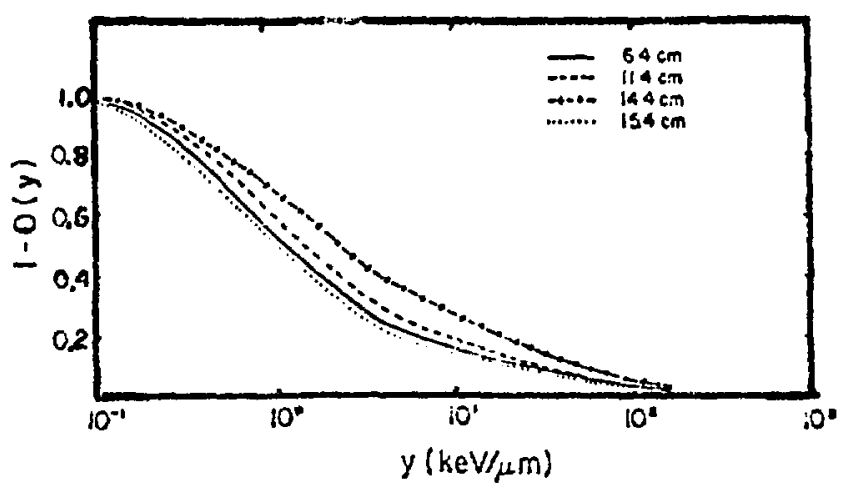

Fig. VIII-4.

Integral dose distributions as a function of $y$ at different depth in the water phantom (see also Fig. VIII-1).

volume, measurements of cell survival as a function of dose at four locations in and near the treatment field have been made. The cell line consisted of $V-79$ Chinese hamster cells. The biological data indicate an RBE of $1.3 \pm 0.2$ at both the peak center and beyond the lateral edges of the beam, and an RBE of 1.1 downstream of the distal edge of the treatment volume. It appears, therefore, as if the RBE outside the treatment volume is nearly the same as the $\mathrm{RBE}$ inside the treatment volume. Hence, the star-produced neutrons will not significantly reduce the dose-localization properties of negative pions.

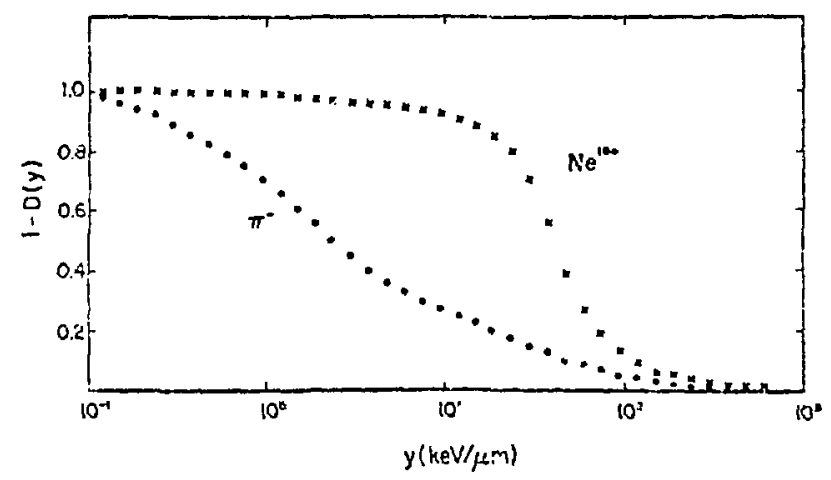

Fig. VIII-5.

Inte'gral dose distributions as a function of $y$ for 80-MeV $\pi^{-}$and 557-MeV/amu $\mathrm{Ne}^{10+}$ beams. The data are taken at $14.4 \mathrm{~cm}$ below the water level and after a 14.5-cm water column, respectively. 


\section{- Therapy Beam Development - LET Measurements (Exp. 273) (LASL)}

Making measurements in the patient at the time of treatment is the ultimate check of pion dose distrioutions and the treatment planning for that pratient. Patient probes have been built which, with the housing, are little cylinders $8-\mathrm{mm}$ wide and 6 $\mathrm{mm}$ high. The detectors proper have an area of about $2 \mathrm{~mm}^{2}$, and the thicknesses are $700,100,50$, and $7 \mu \mathrm{m}$. This enables one to use a thickness appropriate for examining different particles. The LET spectra are obtained in the detector, and these are computer-analyzed to determine doses. Preliminary data from the skin and mouths of several patients are being analyzed.

\section{Treatment Planning}

In conjunction with including tomography data in the dose calculation for patients, an empirical mocel was developed to relate the $\mathrm{CT} x$-ray data to pion stopping power and physical density ratios for human lung, fat, muscle, and soft and hard bone. The model is based upon the assumption that electron density ratios are equal to $\mathrm{x}$-ray linear attenuation coefficient ratios. This assumption tends to fail for low-energy $x$ rays, but the model does account for pion mass stopping power variation in human tissue which is not linear with electron density.

Data from EMI and Ohio-Nuclear CAT-scan machines have been decoded from magnetic tapes. Tomography data for patients are now being routinely processed with the Biomed computer so that a tomography file for each patient is set up as one subfile of a compiete case file for that patient. The CAT-scan data were also used in a new program (CTBILD) that calculates the required bolus for a given beam and patient anatomy. The life-size prints of the bolus design then serve as templates for the actual bolus construction.

The problems of dealing with a possible $4 \times 10^{6}$ words ut CAT-scan data for each patient (equivalent to two complete scans) necessitated the develop- ment of a complete file system in which all data pertaining to a given patient will comprise a single case file. The file system consists of a header containing pointer words to various subfiles where the patient's case information, CAT-scan data, treatmentvolume contours, data for various plans and dose distributions, and radiotherapist's comments are contained. The subfiles are organized by type reference, and version numbers, and allow for the comparison of several plans and beams within a single case file. The flexibility of the file system is such that it can be expanded at any time to include additional subfiles without the loss of any previous capability.

Using the RSX version of PIPLAN at the Biomed $11 / 45$, a case file is generated on magnetic tape that will contain the CAT-scan data and the treatment volume and surface contours. This tape is then read into the CCF version of PIPLAN (on a CDC-7600) through a bit-translator and unpacking program that loads the CAT-scan data directly into the three-dimensional density matrix in large-core memory. This array is then used by the calculational overlay in constructing the dose distributions for a given plan. The CAT-scan data is averaged and packed into the 60-bit CDC word such that four pixels are averaged per cell and six cells are packed per CDC word. The calculation then proceeds as before, utilizing the CAT data for the density at a point.

\section{-Techniques for Materials Identification and Analysis (Exp. 51) (LASL)}

Preparations for Exp. 51 are proceeding. In this experiment, our goal is to demonstrate that it is possible to do isotopic discrimination for high $-Z$ elements with muonic $x$ rays. The experiment will consist of measurements of the $\mathrm{x}$-ray yields from $\mathrm{PuO}_{2}$ samples having differing amounts of the various plutonium isotopes. We have started a series of calculations of the hyperfine splitting in isotopes of plutonium muonic $\mathrm{x}$ rays in order to determine which lines are the best candidates for identifying a given isotope. 


\section{- Muonic X-Ray Analysis Studies (Exp. 100)} (LASL)

Data analysis for Exp. 100 is essentially complete. There were three independent measurements analyzed during this quarter. In the first, we compared the total oxygen to carbon Lyman series ratios for two samples of normal human liver with the same ratios for two cirrhotic liver samples. The differences are shown in Table VIII-Il. These results show that the change of normal soft tissue to a pathological state can be manifested in measurable changes of the relative concentrations of the major eleinents.

Also of interest was the question as to whether muonic $x$-ray yields could provide a tool for monitoring the mineral composition of bone. Therefore, two plastic simulated bone samples were prepared, having roughly the same concentrations of phosphorous and calcium as normal bone. The second sample had $37 \%$ more phosphorous and $30 \%$ more calcium than the first sample. We measured the ratio of the relative yields of phosphorous and calcium Lyman series $x$ rays in sample \#2 to the vields from sample \#1 and found that the measured ratios were consistent with the values one would expect, based on the known concentration ratios between the samples. Muonic $\mathrm{x}$-ray analysis could, therefore, be used to detect relatively small changes in the mineral content of bones in the body.

The third measurement was to determine the relative strengths of the Lyman series lines from carbon in naphthalene and paraffin. Other measurements ${ }^{1}$ have shown that if hydrogen is bonded to carbon, there is a marked effect on the relative strengths of the lines. We asked the question as to whether there is a systematic trend in the line strengths as the number of hydrogen atoms in a

\section{TABLE VIII-II}

\section{TOTAL OXYGEN/CARBON LYMAN SERIES YIELD RATIOS}

\begin{tabular}{|c|c|c|}
\hline Sample & Normal Liver & Cirrhotic Liver \\
\hline$\# 1$ & $1.24 \pm 0.01$ & $1.16 \pm 0.01$ \\
\hline$\# 2$ & $1.24 \pm 0.01$ & $1.17 \pm 0.01$ \\
\hline
\end{tabular}

hydrocarbon molecule is increased. Our results are shown in Fig. VIII-6, along with the results of measurements on diamond. ${ }^{1}$ It is seen that as the $\mathrm{H} / \mathrm{C}$ ratio increases, the strengths of the higher nembers of the series increase. The $\mathrm{H} / \mathrm{C}$ ratio for paraffin is the same as for polyethylene, which was also measured earlier. I Our paraffin results are consistent with the polyethylene results. There are still gaps to fill in, for example, a $\mathrm{H} / \mathrm{C}=1$ system and a $\mathrm{H} / \mathrm{C}=4$ system. We are planning these measurements.

\section{Muon Spin-Rotation Program}

Work is proceeding rapidly on the design of a high field $(5-\mathrm{kG})$ uniformity ( 1 part in $10^{5}$ over a $3-\mathrm{cm}$ by $3-\mathrm{cm}$ cylinder) Helmholtz coil for use in solid-state magnetic studies. The basic coil design is complete, and a power supply from the LAMPF power supply pool has been set aside for use with the coil. Group MP-8 has sent out a request for bids for conductor, and the coil drawings and specifications are being prepared. We are doing the final calculations which will enable us to specify the tolerances for the coil radii. The coil has an inner diameter of $58 \mathrm{~cm}$, an outer diameter of $89 \mathrm{~cm}$, and a physical gap of $21 \mathrm{~cm}$.

\section{-Proton Axiai Tomography (Exp. 286) (LASL)}

Data have been taken for two complete computed tomographic (CT) scans using the experimental layout shown in Fig. VIII-7. A specially developed tune of the $\mathrm{P}^{3}$ channel provided protons in a momentum bite of $0.2 \%$ with a $1.5-\mathrm{mm}$-wide by 3 $\mathrm{mm}$-high beam spot. The energy lost by each proton in the waterbath and phantom was determined from the measurement of the residual proton energy with a hyperpure germanium (HPGe) detector. The HPGe detector had a diameter of $3.3 \mathrm{~cm}$ and a thickness of $1.25 \mathrm{~cm}$. The event trigger was obtained from the two scintillation counters, S1 and $\mathrm{S} 2$. The $\mathrm{S} 2$, with an active diameter of $2 \mathrm{~cm}$, restricted the events to the central region in the HPGe detector. A delay line readout multiwire proportional chamber (MWPC) was used to measure the transverse deflection of the proton at 


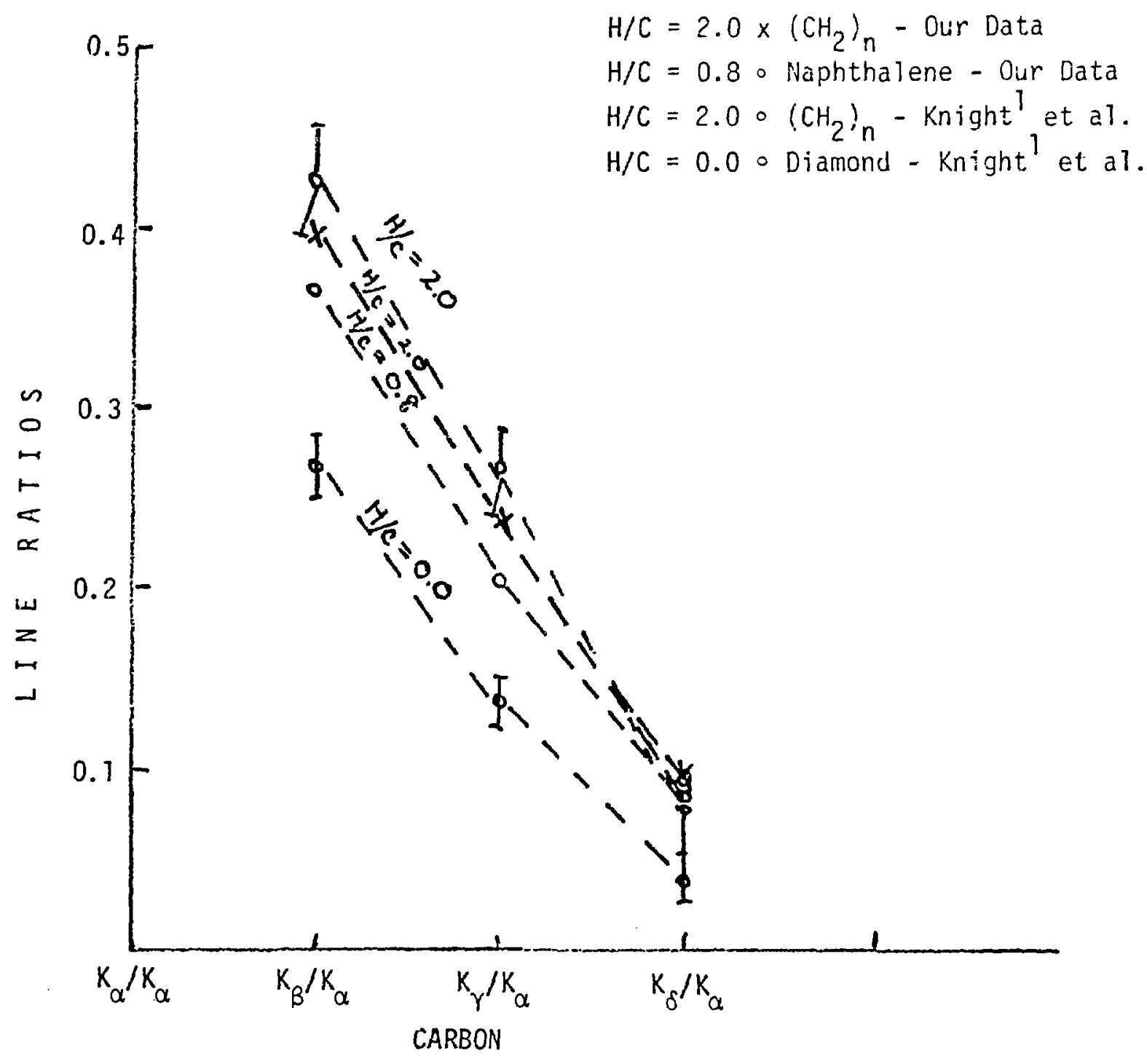

Fig. VIII-6.

Lyman series tine ratios in carbon compounds.

the exit of the waterbath. During the measurements, the phantom was moved through the waterbath across the beam by computer command before a new traverse was begun. The use of buffered CAMAC analog-to-digital converters allowed us to acquire data at an average rate of 750 events $/ \mathrm{s}$ with $50 \%$ deadtime.

For a $30-\mathrm{cm}$-diam phantom, $\sim 675$ events were taken at intervals of $1.25 \mathrm{~mm}$ and $1^{\circ}$. In all, 62.5 million events were recorded in a total running time of $45 \mathrm{~h}$. Measurements indicated that because of nuclear scattering and multiple Coulomb scattering in the phantom and water, only $28 \%$ of the incident protons produced a trigger. The average dose. calculated for a $1-\mathrm{cm}$-thick slice in order to compare it with typical $\mathrm{x}$-ray scanner geometries, is $0.6 \mathrm{rad}$. This cose is based on the number of incident protons needed to produce 62.5 million good events in our geometry, but does not include electronic. computer, and scanner deadtimes.

In the data armiysis, the events at each scan point were binned in eight $2-\mathrm{mm}$ intervals according to the proton position in the MWPC. The HPGe energy spectra in each of these bins were fit by a 


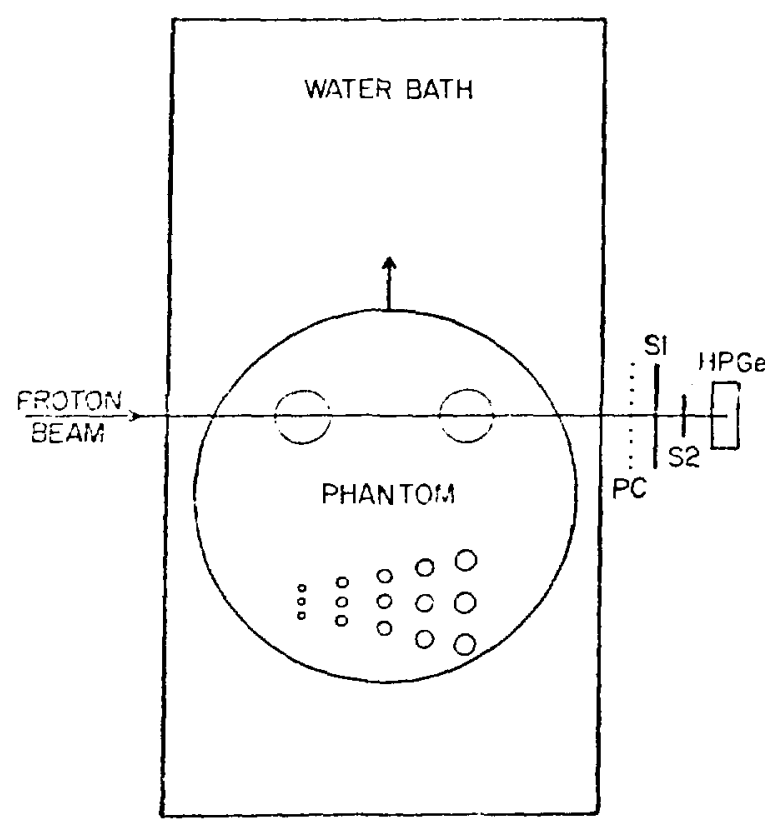

Fig. VIII-7.

Plan vieu of experimental setup. During the ('T scans the phantom us translated and rotated while the proton beam remained stationam:

Gaussian function. The mean residual energy thus determined was then expressed in terms o: the mean residual range. Calibration data were taken is the waterbath at each end of each transverse scan. The two-dimensional distribution of relative stopping power in the phantom was reconstructed from the proton projection data using a filtered backprojection algorithm. The three high-contrast resolution sections of the reconstruction, Fig. VIll-8, show that the proton $\mathrm{C} T$ scan has a resolving power of $2.25 \mathrm{~mm}$, i.e., it can resolve 2.25 - $\mathrm{mm}$ holes on 4.5 mm centers at a contrast of $\sim 15 \%$. The low-contrast section at the bottom of the figure consists of holes in the normal-density polyethylene filled with highdensity polyethyiene plugs. The proton reconstruction can resolve $4.8-\mathrm{mm}$-diam holes at a density difference of $1.8 \%$.

For comparison, the same phantom was scanned on an EMI5005 $x$-ray scanner at the normal 20-s

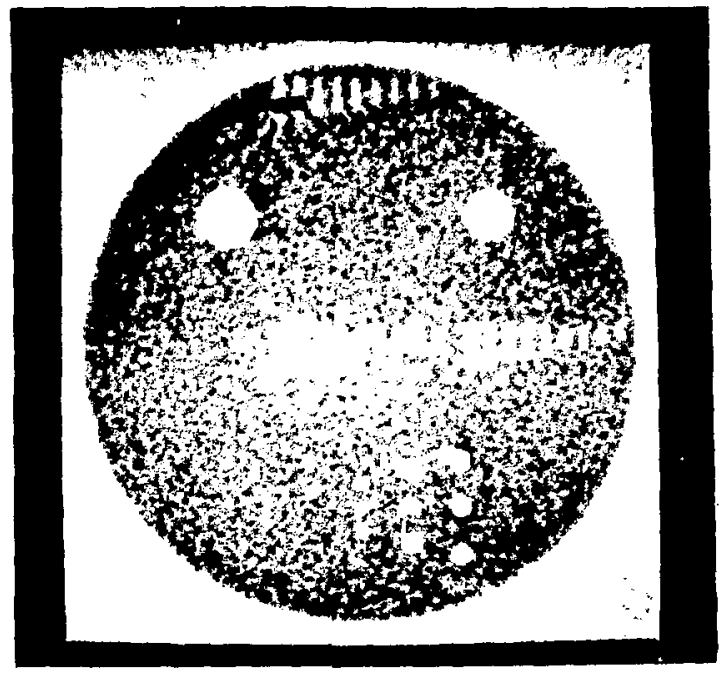

Fig. VIII-8.

Proton CT reconstruction of the 29-cm phantom obtaincd at an equivalent average dose of $0.6 \mathrm{rad}$ in a $1-\mathrm{cm}$-thick slice.

speed. The average dose for such a scan is $2.2 \mathrm{rad}$ and the peak skin dose is $5.5 \mathrm{rad}$. The F.MI highcontrast resolving power was found to be $1.5 \mathrm{~mm}$. somewhat better than the proton scan. However. the low-contrast resolving power is approximately the same as the proton scan. Thus, we conclude that our proton scan of the $30-\mathrm{cm}$ phantom has achieved about the same density resolution as the E.MI scan at a dose which is nearly four times less.

Work is continuing on the analysis of scan data taken for a $20-\mathrm{cm}$-diam phantom. Furthermore. data taken with two different types of detectors in place of the HPGe must be analyzed to evaluate their applicability to faster scans.

\section{REFERENCE}

1. J. D. Knight, C. J. Orth, M. E. Schillaci, R. A. Naumann, H. Daniel, K. Springer, and H. B. Knowles, Phys. Rev. A13, 43-53 (1976). 


\section{LINAC TECHNOLOGY}

Siummarizes work being performed under the auspices of the National Cancer Institute, Division of Cancer Research Resources and Centers of the Department of Health. Education, and Welfare. I

\section{PIGMI Prototype}

\section{Controls Hardwarc - Design}

The basic configuration of the data-acquisition and control system for PIGMI is shown schematically in Fig. IX-1. Its primary components include a central minicomputer, which is connected tor a radial array of microprocessor chassis (MPC) via fast parallel communication links. One MPC is connected by a serial fiberoptic link to a second

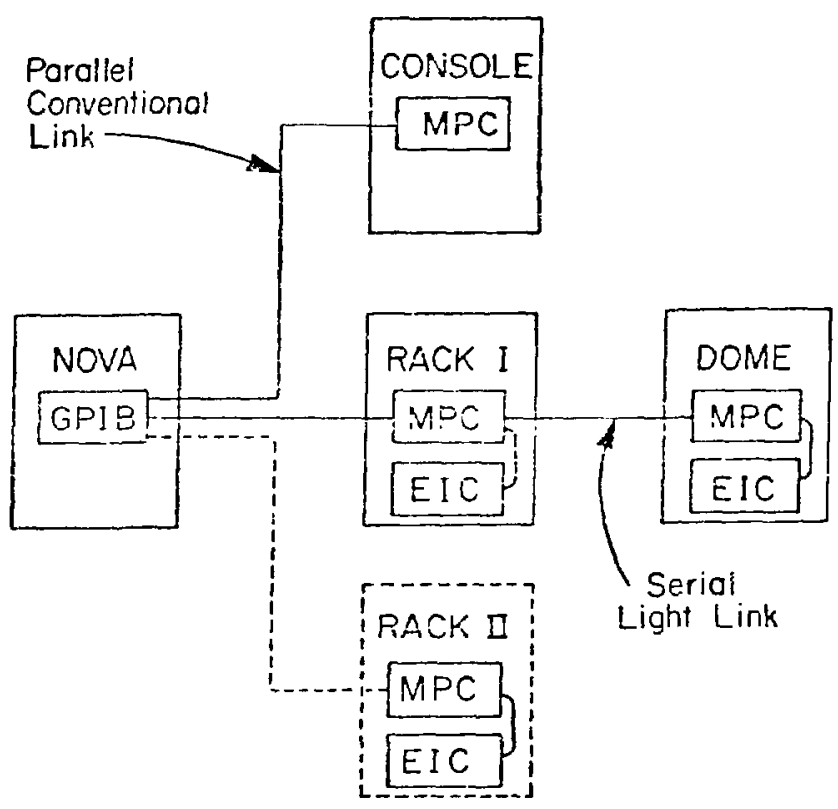

Fig. IX-1.

Schematic of PIGMI control system configuration $(G P I B=$ general-purpose interface board, $M P C=$ microprocessor cassis, $E I C=$ equipment interface chassis).
MPC !ocated in the high-roltage dome. The MPCs are in turn linked to an equipment interface chassis (FIC).

The Console MPC provides dedicated support for the cossole, just as the Dome MPC/ElC controls only the injectur. The Rack I MPC/EIC. in addition to serving as a communication link to the Dome. will provide general control support for the beam transport elements, the accelerator, and all diagnostic devices. As demands on this initial system increase. the Rack II MPC/EIC will be brought into service.

The ElC's perform the jobs of actually controlling acceleratur hardware and collecting data. They operate under the direct control of an MPC. which arts as a semiautonomous processor. The MPCs may perform control functions independently. but they will also receive requests from the central computer to perform certain control functions. The MPCs may also be requested to transmit information to the computer.

Fach FIC will contain some subset of the modules described helow, as required by the associated equipment.

1. Binary Command Modules (BCM) provide:

a. eight low-power eontact closures,

b. eight corresponding enable interlocks, and c. eight corresponding "latch-back" data bits.

2. Binary Data Modules (BDM) return:

a. thirty-two channels of binary data packed into four 8-bit bytes.

3. Analng Command Modules (ACM) provide tither:

a. iwo 12-bit DAC channels, or

b. four 8-bit DAC channels.

4. Analog Data Modules (ADM) may include any subset of the following features:

a. one 16-channel, 12-bit A/D converter.

b. one 16-channel multiplexer.

c. sixteen channels of sample-and-holds, and

d. sixteen preamplifiers with timed data capability and resistor selectable gaini.

5. Stepping Motor Modules (SMM) provide;

a. four channels of low-level drive pulses,

b. four 12-bit channels for position readout. and

c. four motor-enable interlocks. 
Each MPC will contain a minimum set of cards which provide the following functions: 1) crate controller (CC); 2) MC6800 microprocessor unit (MPU); 3) asynchronous interface (ACIA); 4) four channels for peripheral interface (PIA), two required; 5) MPC front-panel controllers, four cards; 6) $4 \mathrm{k}$-byte ROM/RAM memory; and 7) first-in/firstout communications buffer (FIFO).

In addition, an MPC may contain additional cards from the following list as required by the hardware to be controlled: 1) storage scope driver, 2) plotting software in ROM, 3) 8k-byte RAM memory, 4) up/down counter, and 5) period counter.

\section{Controls Hardware - Status}

Most of the basic components of the initial system as shown in Fig. IX-1 (excluding Rack II) are either installed or in hand awaiting final checkout. Each MPC contains at least the minimum set of cards, and each has been thoroughly exercised. Approximately $90 \%$ of the remaining MPC cards required for initial operation are in hand. Development work on EIC modules is $50 \%$ complete. Binary modules (BCMs and BDMs) are either in fabrication or on hand; only prototypes exist of the analog and stepping motor modules (ACMs, ADMs, and SMMs). The system console is nearing completion, with half of the interactive tools already operational and the rest awaiting completion of their interfaces.

\section{Controls Software}

The system described above requires sophisticated software support in both the crntral computer and MPCs. A real-time disk operating system (RDOS) was purchased, along with a NOVA 3 that serves as the rentral computer. By exploiting some of the special features of RDOS, a subsystem has been designed to run under RDOS and provide all of the necessary message-handling for communication with MPCs. Incoming messages are processed by the subsystem on interrupt, and the information is passed to the expectant task while outgoing messages are cued for high-priority transmission. The system allows a large number of application tasks to run asynchronously, each sending and receiving messages to MPCs.
The basic operating system for all MPCs is the same, and contains both a polling loop and an interrupt service sequence. Device drivers as written in modular form and can easily be added to either the polling loop or interrupt sequence, thus allowing a system to be easily individualized to service the required hardware.

To facilitate efficient communication between the computer and MPCs, a message protocol has been established that makes use of 25 unique message codes. These message codes identify to the receiver the data which comprises the rest of the messages. Incoming messages are handled on interrupt by the MPC system which puts the data into cues to be acted upon by software modules in either the polling loop or on software interrupt. Outgoing messages are cued for transmittal by modules in the polling loop.

All of the basic features of the software described above are operational in both the computer and the console MPC. These systems now support one-third of the message codes envisaged for communication throughout the system, and a modest library of FORTRAN callable routines has been written and tested which support all currently existing console hardware. Initial application programs indicate that the control system will be convenient to use for accelerator development, and that one may expect excellent response.

\section{PIGMI Prototype Quadrupole Magnets}

The linac portion of the PIGMI prototype consists of a single tank loaded with 64 drift tubes, which will accelerate a proton beam from 0.25 to $7.32 \mathrm{MeV}$ in a total length of $2.48 \mathrm{~m}$ at a frequency of $450 \mathrm{MHz}$. The first 28 cells will have no quadrupole lenses and will be entirely dependent on the alternating-phase-focusing (APF) principle for beam confinement. Permanent-magnet quadrupole (PMQ) lences will he 'acinduc ' 'ito every other drift tube in a $+0-0$ configuration in the remaining 36 cells. The quadrupole gradients will vary in strength from $0.95 \mathrm{kG} / \mathrm{cm}$ to $7.00 \mathrm{kG} / \mathrm{cm}$. The PMQ ideally would satisfy the field requirements, have hyperharmonic components $<0.5 \%$, and be inexpensive and amenable to easy fabrication and tuning. The application of circular pole PMQ using HICOREX-22 would appear to be most promising. 
This part of the program is headed by Mr. Norio Saito, a guest scient ist from Hitachi, Ltd. of Japan.

Ten new yoke geometries for the circular pole PMQ were fabricated to support the effort to minimize the higher order harmonic content. A prototype PMQ, using trapezoidal magnetic pole bases and PANDIRA-designed soft iron pole tips, was completed (see Fig. IX-2).

Beginning with circular poles having a bore field in excess of that required, the PMQ can be tuned to the required field by grinding a flat on the bore side edge of each of the poles perpendicular to the pole center-bore center line. The presence of the flat not only reduces the quadrupole field gradient but also reduces the hyperharmonic field components for a net improvement in the quality of the lens. PANDIRA calculations, confirmed by experiment, show the decrease in quadrupole field gradient is slow compared with the decrease in harmonic content. A representative plot of the variation of the quadrupole field gradient and the 6th and 10th harmonic content with flat depth is shown in Fig. IX-3. The pole radius-to-bore radius ratio $(\mathrm{P})$ is 1.00 . The bore radius is $6 \mathrm{~mm}$ and $N \equiv t / 0.279$ where $t$ is the height of the segment removed in millimeters.

The harmonic content is expressed in percentage of the quadrupole harmonic. A PANDIRA plot of the flux lines for this example is shown in Fig. IX-4.

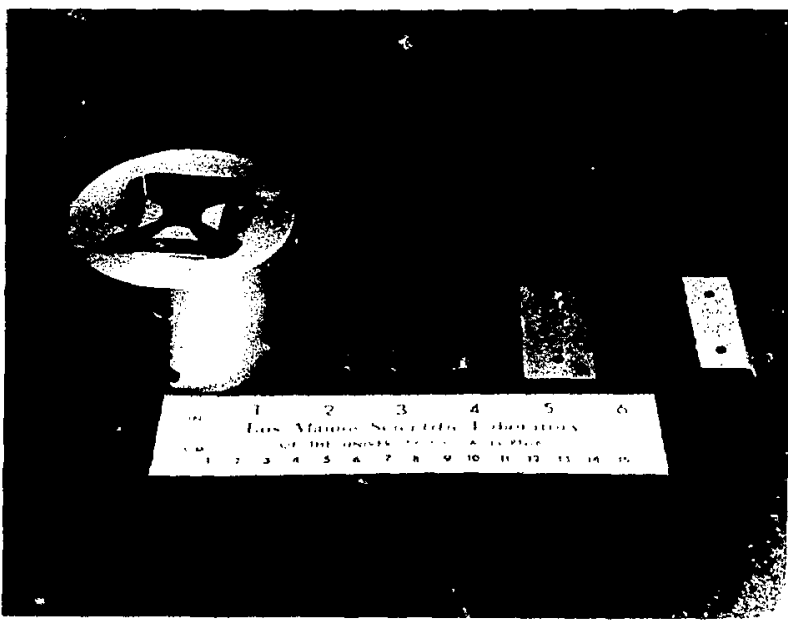

Fig. $I X-2$.

PIGMI permanent-magnet quadrupole.

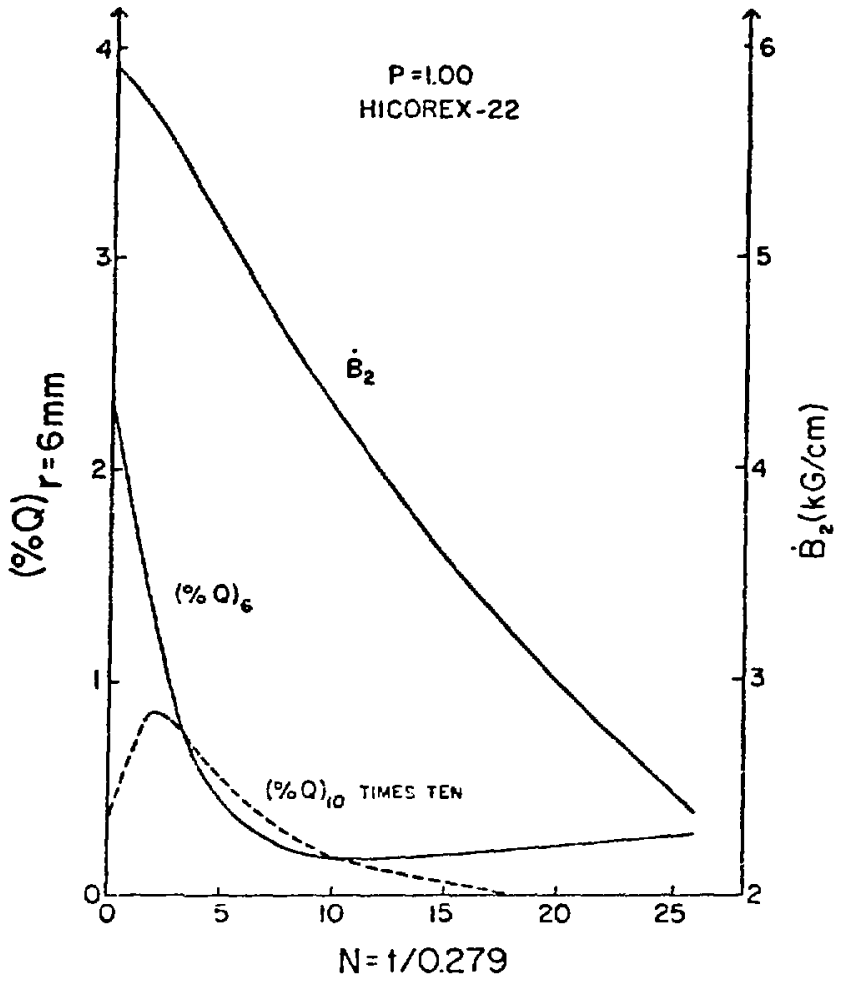

Fig. IX-3.

Plot of quadrupole field and harmonics (6th and 10th) as function of flat depth.

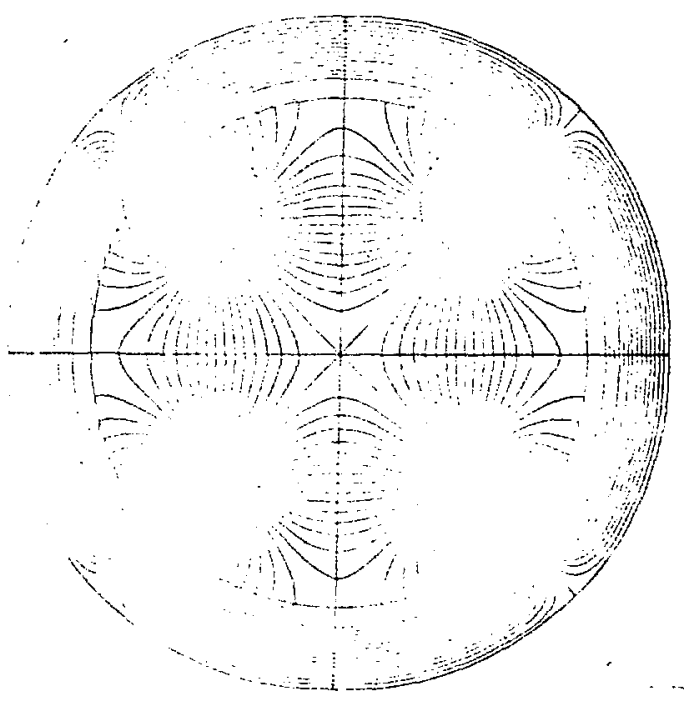

Fig. IX-4.

PANDiRA plot of shaped circular-pole quadrupole magnet. 


\section{PIGMI Prototype - Mechanical}

A component layout of the PIGMI prototype accelerator and experimental area has been prepared and is being used for planning and device placement in ETL. The injector area has been cleared and installation begun. The $250-\mathrm{kV}$ C-W source has been assembled and installed. The injector shield cage has been procured; following the completion of the cage installation, high-voltage testing will begin.

The injector vacuum manifold is being fabricated, with completion expected in mid-December. The ion source/accelerating column mounting stand has been designed and released for fabrication, with an expected delivery in late December. A LeyboldHereaus turbomolecular vacuum pump is being procured for use with the injector. Its high pumping capacity at the planned operating pressure, lack of contaminants, low vibration, and minimal cooling requirements make it especially attractive for a nonlaboratory environment.

The stem terminations for the initial drift tubes (up to $\sim 2.5 \mathrm{MeV}$ ) have been designed, and a prototype is expected to be available for testing early in the next quarter. The stem termination has an outside diameter of $4.44 \mathrm{~cm}$. Metex is used to provide the rf seal, and all-metal vacuum seals will permit bakeout in situ. Positioning in the $\mathrm{z}$-axis is accomplished by precision machining of the $\mathrm{rf}$ cavity. Its $2.40-\mathrm{cm}$ wall thickness and relatively small diameter $(\sim 45 \mathrm{~cm})$ should provide an accurate and stable platform. Radial positioning is accomplished via a system of jackscrews. The termination design is such that the metal vacuum seals can be replaced without disturbing the drift-tube alignment. An external Viton 0-Ring sealed cap will provide for leak detection and soft vacuum pumping. Three stems of $1.6-\mathrm{mm}$ diam will be used to support these initial drift tubes. Because of the compactness, a three-step, ten-degree offset sequence will be employed. Two of the stems will be used for the drift-tube water-cooling supply and the remaining for the return. Allowing a $5^{\circ} \mathrm{C}$ rise in water temperatures, calculations show the stem flow as still being laminar. The drift tubes subsequent to about the $2.5-\mathrm{MeV}$ point will be supported vertically by a single stem of a design similar to that employed in PIGLET.
The first APF test cavity with drift tubes installed is shown in Fig. IX-5 in preparation for beadperturbation studies.

A test cavity for the three-stem configuration is currently being fabricated. This cavity will permit vacuum testing of the drift tube and its stem support hardware before installation in the PIGMI prototype tank. It will also serve as a learning vehicle for mounting and aligning drift tubes in a noncritical environment.

\section{PIGLET}

All of the component parts for PIGLET, the sixcell, $45-\mathrm{MeV}$ drift-tube geometry, field-gradient test cavity, have been delivered and assembly has begun. The PIGLET vacuum tank has been brightcopperplated by an outside vendor (see Fig. IX-6) and is ready for rf measurements at the ETL Building. The drift tubes and heads will be brightcopperplated at LASL.

The completed PIGLET rf vacuum window assembly is shown in Fig. IX-7. This unit has been tested and will require some modification to cbtain proper rf characteristics.

Following the assembly of PIGLET, the cavitycoupling iris will be sized experimentally to provide

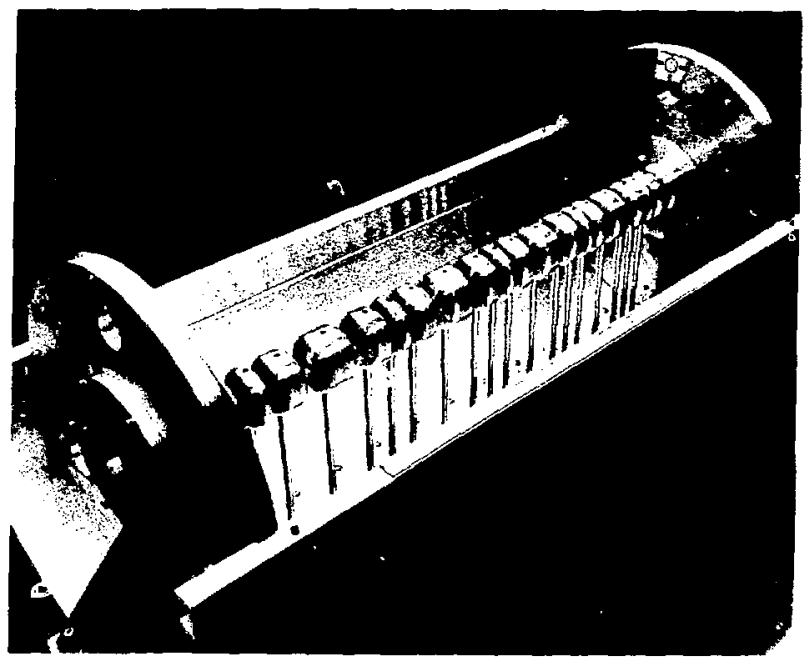

Fig. IX-5.

APF beadpull model. 


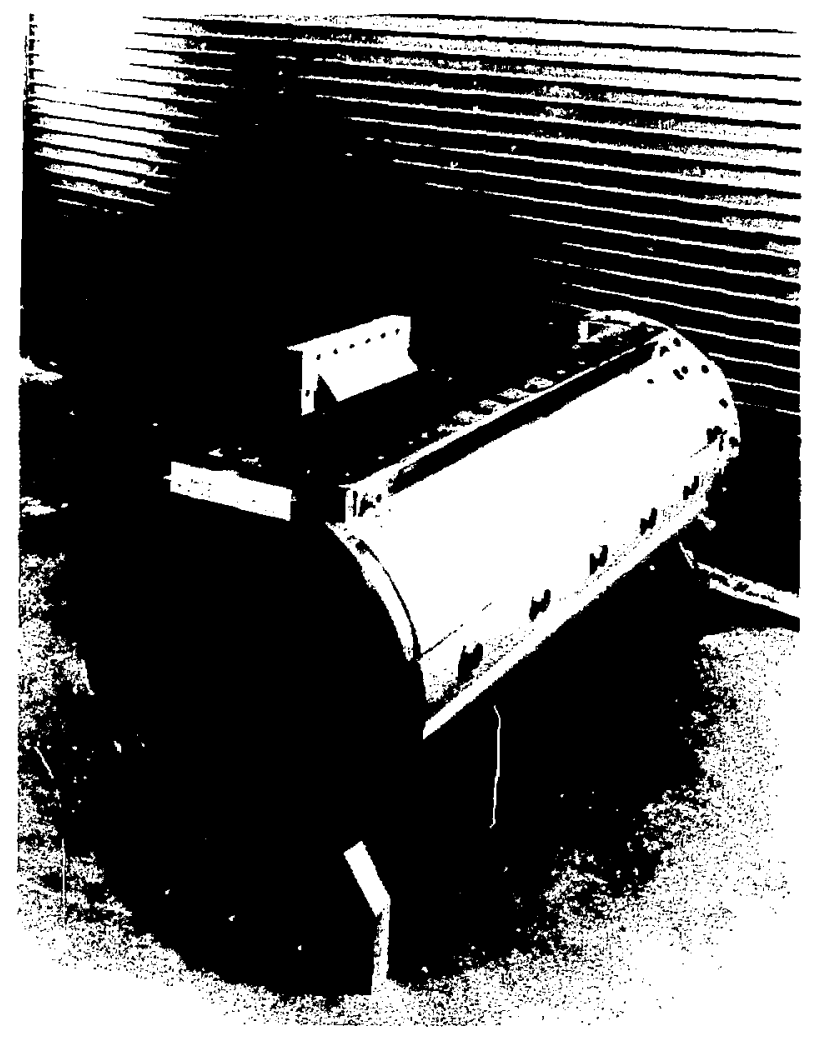

Fig. IX -6 .

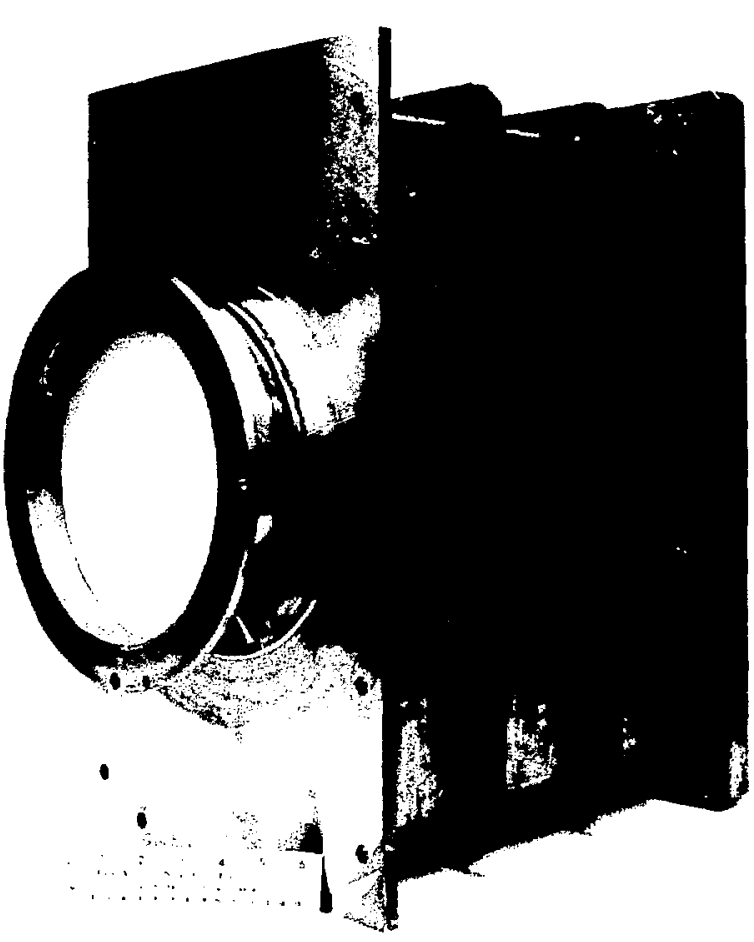

Fig. IX-7.

PIGLET rf window assembly.

PIGLET vacuum tank bright-copperplated.

a flat match to the WR-1800 waveguide preparatory to powering the cavity. The vacuum window originally designed, using an available ceramic disk to interface between the cavity and the klystron drive line, proved to have an intolerably narrow bandwidth when matched. A revised window design using Rexolite as the dielectric element is in the shop and will be used in the interim pending the location of a suitably large-diameter ceramic disk. We hope to bring PIGLET up to power during the next quarter.

\section{PIGMI Injector}

The ion beam trajectory code SNOW (see Sec. II) has been used to check calculations on the convergent beam geometry for the PIGMI injector. Figure IX-8 shows the equipotentials and trajec- tories calculated for the convergent electrode design when a zero-temperature, uniform-density ion beam is injected into the plasma. The irregularity of the equipotentials at the column exit is due to the grid size used in the calculation. The grounded drift tube beyond the exit aperture was used to simulate the buncher aperture. The results given by this calculation confirm the results of the numerical calculation ${ }^{1}$ used in the design of the electrodes. The $50-\mathrm{mA}, 8-\mathrm{mm}$-diam beam extracted from the plasma converges to a $4-\mathrm{mm}$-diam beam beyond the exit aperture when the acceleration voltage is $250 \mathrm{kV}$.

Work has continued on the PIGMI injector. The injector support stand and vacuum manifold are now being fabricated, and assembly is planned to start at the end of December. The ion source has been refurbished and preparations for final beam tests are now in progress. The spherical diode design will be experimentally verificd. 


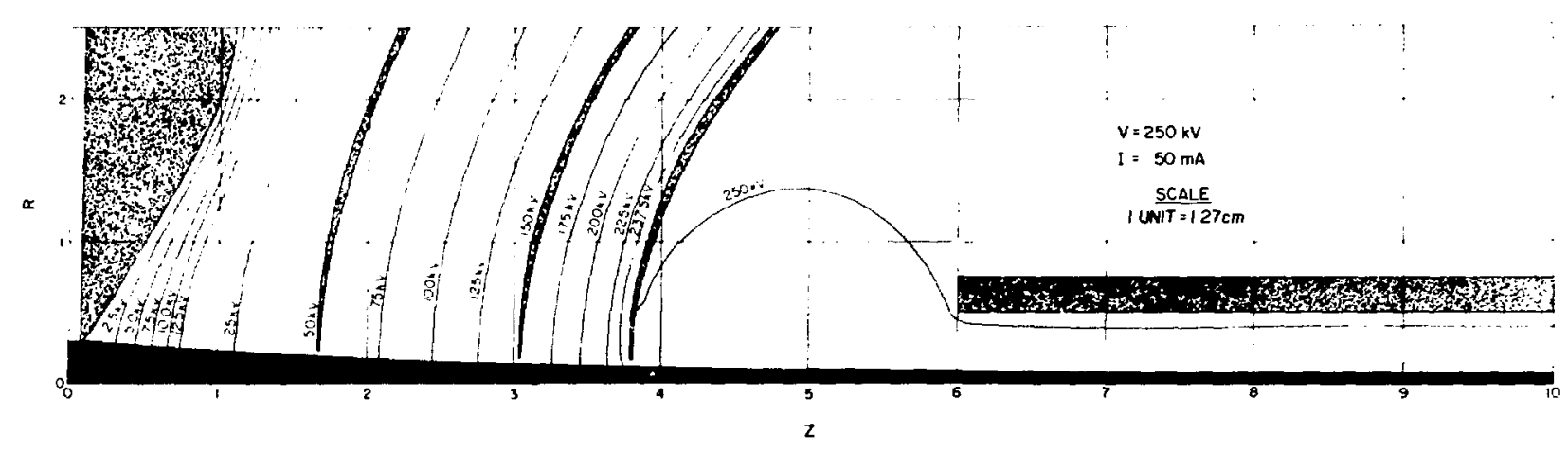

Fig. $I X-8$.

Ion trajectories in the $250-k \mathrm{~V}$, spherically convergent design for the PIGMI injector.

\section{The Gyrocon}

Studies have continued on a new microwave amplifier device, called the gyrocon by its inventors, which appears capable of extraordinarily high efficiency. A schematic diagram of the amplifier is shown in Fig. IX-9. The beam is deflected by a rotating magnetic of field in the $\mathrm{TM}_{110}$ deflection cavity. It is further deflected by the fringe fields of a conical bender solenoid, passes through a set of vertical focus coils, and enters the resonant-ring output cavity. The phase velocity of the fields in the resonant ring is designed to be equal to the deflection velocity of the beam, and, if the field amplitude and phase are proper, most of the beam's kinetic energy can be converted into rf power as the electrons are decelerated by the rf fields. The beam leaves the rf region and impinges on a collector. A mathematical model of the device was made and uevd to determine the overall dc-to-rf conversion efficiency. Some of the results are summarized in Table IX-I below for a $450-\mathrm{MHz}$ amplifier which has a $10-$ deg deflection cavity. In the table, $E_{0}$ is the field strength in the output waveguide, $n_{e}$ is the electronic efficiency, $P_{1}$. is the output waveguide loss, $P_{o}$ is the output power, $V_{0}$ is the voltage, $I_{0}$ is the beam current, $G$ is the gain, and $\eta$ is the overall dc-to-rf conversion effïciency.

The input beam power was set at 100,300 , and $1000 \mathrm{~kW}$. Only the cases with an overall positive conversion efficiency are listed. Three important trends shown are that the electronic efficiency can easily exceed $90 \%$, the overall efficiency can exceed $80 \%$, and the overall efficiency increases with the dc beam power. Since the power losses in thr output waveguide are tens of kilowatts, the beam power must be at least $100 \mathrm{~kW}$ for efficient operation. The device does promise to be a very efficient, but rather low gain, rf amplifier. A further advantage of the gyrocon is that very high output powers appear to be achievable, which would reduce the capital costs of a large rf system.

The bender system was not included in the calculations of Table IX-I, but the analysis program has been improved to calculate the threedimensional trajectories in a solenoidal bender magnet. An example of the bender results is shown

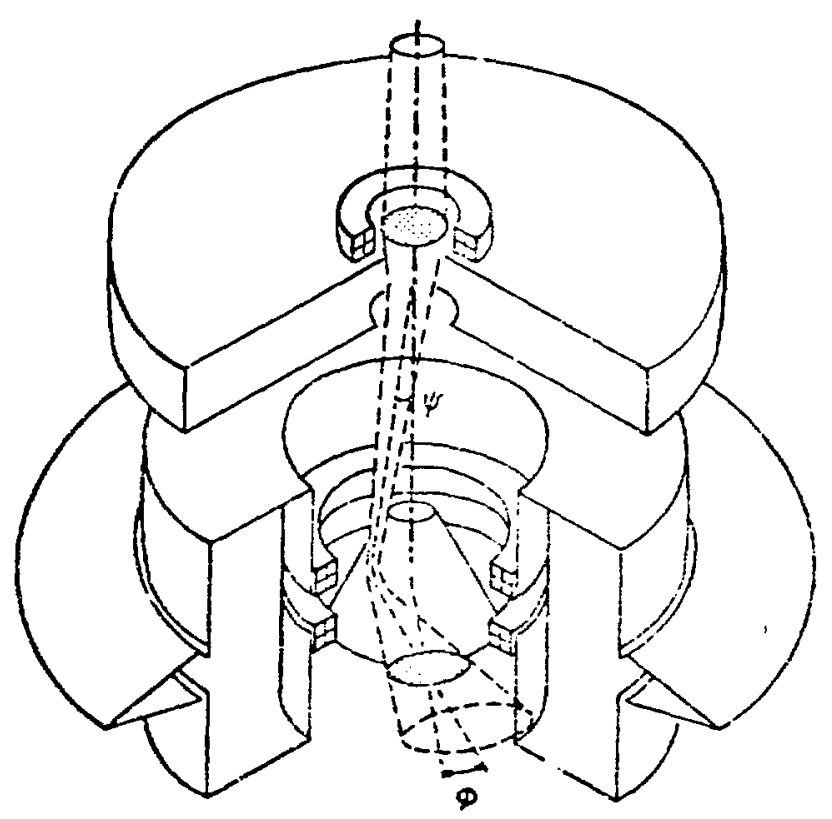

Fig. IX-9.

Schematic drawing of gyrocon. 
TABLE IX-I

10-DEGREE GYROCON RESULTS AT $450 \mathrm{MHz}$

\begin{tabular}{|c|c|c|c|c|c|c|c|}
\hline$V_{0}(k V)$ & $I_{0}(\mathbf{A})$ & $\mathrm{E}_{0}(\mathrm{MV} / \mathrm{m})$ & $\eta_{\mathrm{e}}$ & $\mathbf{P}_{\mathrm{L}}(\mathbf{k W})$ & $P_{0}(\mathbf{k W})$ & $G(\mathrm{~dB})$ & $\eta$ \\
\hline 50 & 2.0 & 1.20 & 0.940 & 15.8 & 78.2 & 26.9 & 0.7664 \\
\hline 100 & 1.0 & 1.65 & 0.956 & 31.7 & 63.8 & 13.9 & 0.6223 \\
\hline 200 & 0.5 & 2.30 & 0.935 & 68.5 & 25.0 & 8.2 & 0.2408 \\
\hline 50 & 6.0 & 1.50 & 0.796 & 24.0 & 214.7 & 31.3 & 0.7110 \\
\hline 100 & 3.0 & 1.85 & 0.946 & 38.8 & 245.6 & 19.8 & 0.810 \\
\hline 200 & 1.5 & 2.50 & 0.935 & 76.9 & 203.6 & 17.3 & 0.670 \\
\hline 100 & 10.0 & 1.75 & 0.890 & 35.7 & 854.2 & 25.2 & 0.852 \\
\hline 200 & 5.0 & 2.70 & 0.931 & 87.3 & 843.4 & 23.5 & 0.840 \\
\hline 500 & 2.0 & 4.10 & 0.877 & 234.1 & 642.8 & 18.9 & 0.638 \\
\hline 1000 & 1.0 & 6.80 & 0.815 & 658.9 & 155.9 & 9.1 & 0.153 \\
\hline
\end{tabular}

in Fig. IX-10. The contour lines have 50-G separations, and the uniform conical solenoid is approximated by eight separate coils. The geometry corresponds to the $181-\mathrm{MHz}$ Novosibirsk gyrocon operated at $210 \mathrm{kV}$. Five electrons, uniformly spaced in a circle at the start of the calculation, are followed through the magnetic fields of the conical solenoid and the two vertical focus coils.

The bender solenoid has large aberrations, but these are at least partially compensated by the vertical focus coils. The beam in Fig. IX-10 has an extent of $20^{\circ}$ in the azimuthal direction. Space charge is neglected in all the calculations so far, so the calculated beam sizes are optimistic estimates. The gyrocon analysis code is being modified to take the output results from the bender calculations and recalculate the dc-to-rf conversion efficiency with those output results as input quantities to the $\mathrm{rf}$ calculation.

Cold test models of the output resonant ring and the $\mathrm{TM}_{110}$ input cavity were designed, built, and tested. The shielding necessary to keep the output resonator from radiating power through the beam slots was empirically determined. The frequency dependence of the desired mode, and its two nearest neighbors on the slot width, was determined. The deflection cavity had an inadvertent ellipticity of $<1 \%$, and this caused the desired $\mathrm{TM}_{110}$ modes to have different frequencies. Several types of tuners were tried, but none with a sufficiently high tuning range was found. Thus, the deflection cavity will be rebuilt to higher tolerances and retested.

\section{Electronuclear Fuel-Producing Accelerator}

It has been estimated that somewhat over $75 \%$ of the cost of an Electronuclear Fuel-Producing Accelerator (ENFPA) will be associated with the rf systems. Since the cost is so rf-dominated. most of the effort to date has been directed toward obtaining realistic costing data for this portion of the system.

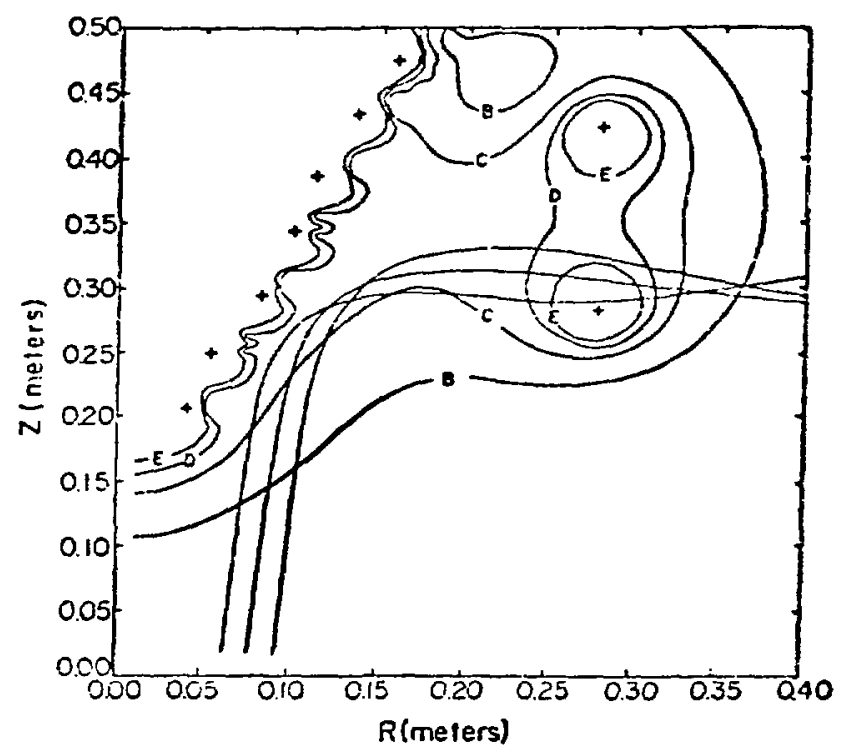

Fig. IX-10.

$R-Z$ projection of three electron trajectories in a gyrocon bender solenoid with 50-G magnetic field contours. 
A contract was released during the current quarter to solicit the background information necessary for a more detailed tube systems study. Matrices covering the cost of $\mathrm{rf}$ power generation as a function of $\mathrm{rf}$ module capacity and frequency for klystrons and gridded devices were sought. These studies have been completed. In the case of the klystron, the matrix can be approximated by the expression:

$$
\text { cost } / \text { module }=0.53 \mathrm{P}^{0.50} \mathrm{~F}^{-0.33} \quad \mathrm{M} \$ / \text { module }
$$

where $\mathrm{P}$ is the unit module power output in megawatts and $\mathrm{F}$ is the frequency in megahertz. The matrix covered $0.25 \leq \mathrm{P} \leq 5.0$ and $50 \leq \mathrm{F} \leq 600$. This yields a cost/watt of:

$$
53 P^{-0.5} F^{-0.33} d / W
$$

The maximum module power output capacity is related to the frequency by the expression:

$$
P_{\max }=612.5 \mathrm{~F}^{-1} \text { for } 50 \leq \mathrm{F} \leq 600 \text {. }
$$

Lastly, the dc-to-rf conversion efficiency is related to the microperveance by:

$$
\eta=0.70-0.06 \mathrm{k} \text {. }
$$

Both of these latter two expressions are somewhat more conservative than those generated in-house. They will, however, be used in subsequent parameter setting and costing inasmuch as they represent what a highly respected klystron manufacturer believes to be compatible with a high probability of developmental and manufacturing success $(>90 \%)$.

Two matrices were submitted by the gridded-tube contractor. The first covered "on-the-shelf" tubes with $0.25 \leq \mathrm{P} \leq 1.0$ and $50 \leq \mathrm{F} \leq 150$, and yielded $\mathrm{A}$ module cost of:

\section{$0.164 \mathrm{P}^{0.70} \mathrm{~F}^{0.04} \mathrm{M} \$ /$ module ,}

with a cost/watt of

$16.4 \mathrm{P}^{-0.30} \mathrm{~F}^{0.04} d / \mathrm{W}$

The maximum module power output capacity was related to frequency by

$$
P_{\max }=50 \mathrm{~F}^{-1} \quad 50 \leq \mathrm{F} \leq 150 .
$$

The overall dc-to-rf conversion efficiency was $\sim 72 \%$ within the given frequency and power limits.

The second matrix covered tubes yet to be developed with $0.25 \leq \mathrm{P} \leq 5.0$ and $100 \leq \mathrm{F} \leq 200$, and yielded a module cost of

$$
0.0326 \mathrm{P}^{0.33} \mathrm{~F}^{0.33} \quad \mathrm{M} \$ / \text { module }
$$

with a cost/watt of

$$
3.26 \mathrm{P}^{-0.67} \mathrm{~F}^{0.33} \mathrm{~d} / \mathrm{W}
$$

Overall efficiencies ranged between 82 and $89 \%$ within the given frequency and power limits! These efficiencies were obtained by utilizing a new tube concept - a harmonically enhanced drive, nonintercepting anode, depressed collector, linear beam gridded tube. This truly exciting concept would appear to be most promising and should be pursued. Its high gain $(\sim 25 \mathrm{~dB})$ would allow it to share the system simplicity of a klystron while its high efficiency makes it most attractive from an operational and ac-to-beam conversion standpoint.

It is hoped that a supplementary tube-study contract can be released during the next quarter to extend this background garnering to include crossedfield devices. It is also hoped that a similar study contract can be written to obtain that information germane to costing a unit power supply as a function 
of output power and voltage. Lastly, an attenipt will be made to generate the unit length costing of drifttube and coupled-cavity (disk and washer) structures as a function of frequency.

\section{REFERENCE}

1. D. W. Mueller, private communication, September 1977. 


\section{MANAGEMENT}

\section{Budget and Personnel Levels}

The FY-1978 budget is held up temporarily, pending action by Congress on the DOE money bill. We expect reso'ition to be forthcoming before the end of the calendar year. The FY-1977 capital equipment funds are obligated or costed, and a start has been made on FY-1978 items.

As a result of the planned shutdown in September, management decisions, and excellent cooperation by both LASL and outside user personnel, there was only a small overrun of operating costs for FY 1977. However, operating costs projected for FY 1978 indicate difficulties in meeting our commitments because of budgetary limitations.

The average number of full-time equivalent ( $F \mathrm{TE}$ ) employees chargeable to the medium-energy physics program is 309. This is a reduction of about 11 people since the end of FY 1977, and an indication of the impact of the tightness of FY-1978 funds.

\section{Safety}

\section{Safety Program}

An important addition has been made to the program this quarter. D.R.F. Cochran, User Liaison and Chairman of the LAMPF Padiological Safety Committee, has agreed to assume the uriect (group leader) responsibility for user safety. Although several methods are used to bring the safety message to users, Dr. Cochran will add others to make this part of the LAMPF Safety Program more effective.

\section{Radiological Safety}

Control of Personnel Exposures. During the two-month shutdown this quarter, major repair work in high radiation areas was accomplished with no overexposures of personnel. The Health Physics staff exercised close control of exposures by following guidelines established by the LAMPF administration prior to shutdown. The maximum weekly dose for personnel was limited to $300 \mathrm{mrem}$. The average dose received during the $e_{i_{r}}{ }^{2} h t$-week shutdown was $100 \mathrm{mrem}$. According to LAMPF policy, operational procedures known as "game plans" were written for 64 jobs. Each game plan was reviewed by the LAMPF Health and Safety Office (HSO) and the LAMPF Hsalth Physics Office. Porket dosimeters were issued in addition to regular film badges, and by careful planning and surveillance, personnel exposures were kept below the established guidelines.

Control of Radioactive Materials. Close control of the movement of radioactive materials at LAMPF continued as a major effort. The gateway monitor (discussed in the previous progress report) detected several unexpected radioactive shipments, and the shipments were directed to controlled areas. $A$ horn and light were added to warn a driver that radioactive material is being carried; the vehicle must be returned to the LAMPF Operations Building to be checked by a health physics surveyor.

The crystal-detector portal monitors placed at 11 critical points throughout the site have picked up radioactivity on personnel. The Radiological Safety Committee met to review the control prograin, and recommended that additional units be procured for other locations.

The disposal of cooling water from the target cells and beam stops has long been recingnized as a problem which must be solved because of the accumulation of tritium. Through a series of meetings among LAMPF, Health Division, and Engineering Department personnel, a practical solution was found, and installation work started this quarter. Basically, a small amount of the radicactive water is continuously withdrawn from the system, passed through ion-exchange columns which remove all but the tritium contamination, and stored in an auxiliary tank. It is then pumped through spray nozzles onto a filter bed in the main exhaust stack and blown out into the atrnosphere. Extensive studies have shown that this disposal rnethod presents no problems to the environment and the levels of tritium in uncontiolled areas will be well below permissible concentrations. Disposal will be controlled so as to select periods of acceptable meteorological conditions. 


\section{Industrial Safety}

In parallel with the major staff efforts to complete repairs and modifications to the accelerator and experimental areas during the shutdown, significant progress was made in clearing up numerous minor electrical deficiencies and OSHA-type industrial safety items.

Safety inspections of all areas at LAMPF were conducted. Daily general surveillar.ce also continued. All deficiencies were reported to the responsible group leader, although many were corrected on the spot with HSO assistance.

\section{LAMPF Visitors Center}

There were 288 research guests at LAMPF during this report period, with 89 checking in and 164 departing. The research guests at LAMPF are listed in the Appendix.

\section{LAMPF Users Group, Inc.}

\section{Liaison Office}

The annual election of officers for the Board of Directors of the LAMPF Users Group, Inc. was conducted by mail ballots sent to 982 menibers of the group on September 21, 1977. The Nominating Committee, consisting of Herbert L. Anderson, Chairman (University of Chicago/LASL), Robert $J$. Macek (LASL), W. Kenneth McFarlane (Temple University), Robert A. Naumann (Princeton University) and Robert T. Siegel (College of William and Mary), prepared the following slate of candidates. No additional candidates were placed on the ballot by petition.

\section{Chairman-elect:}

Robert A. Eisenstein (Carnegie-Mellon University)

\section{Members:}

Isaac Halpern (University of Washington)

Clifford K. Hargrove (National Research Council of Canada)

Virgil L. Highland (Temple University)
Richard E. Mischke (LASL)

Anthony L. Turkevich (University of Chicago)

The two candidates for members of the Board receiving the most votes will replace Barry $\mathbf{M}$. Preedom (University of South Carolina) and Paul W. Todd (Pennsylvania State University), who are completing two-year terms of office. The results of the balloting will be aunounced at the Eleventh LAMPF Users Meeting which wili be held in Los Alamos on November 13-15, 1977.

\section{Buard of Directors and Technical Advisory Panel}

The Board of Directors will meet at Los Alamos on Tuesday evening, November 15, 1977, and the newly elected officers for 1978 will be invited to attend. Agenda items are: recommendations to Louis Rosen for PAC members, report on graduate student survey, repori on changes to by-laws, follow-up on USSR exchange, follow-up on plans for summer schools, appointment of TAP representatives, report on long-range planning, report on plans for library, new attitudes on safety, and the general business session.

The Technical Advisory Panel will meet at Los Alamos on Wednesday, November 16, 197\%. Agenda items will include the following: status report on LAMPF, report on Summer Study on Computing Facilities, funding status of DOE for a group for compilatio: and evaluation of data for mediumenergy physics, summer workshops, report on longrange planning, new attitudes on safety, and a general discussion on the make-up of the TAP.

\section{Quarterly Report}

This Progress Report is in a new format with activities organized by major areas in the facility. Thus reports from different groups on related activities are brought together and edited, which should produce a clearer picture of facility status and progress, make it easier to find specific information, and reduce redundancy. The format is provisional, and will be modified or even discarded depending upon response from those concerned. 


\section{Statistics}

Listed below are statistics of the LAMPF Users Group, Inc. as of October 31, 1977:

\section{Membership}

Non-LASL

LASL

\section{Membership by Institute}

LASL

National or Government labs

U. S. Universities

Industry

Foreign

Hospitals
197

121

391

57

156

1002

TOTAL $\quad$ i002

TOTAL

\section{Number of Institutes}

National or Government Labs $\quad 30$

U. S. Universities 115

Industry 43

Foreign 74

Hospitals 49

TOTAL 311

\section{Regional Breakdown}

East (PA, NJ, DE, WASH DC, MA, NY, CT, VT, RI, NH, ME) 144

Midwest (OH, MO, KS, IN, WI, MI, II, ND, SD, NB, IA, MN)

South (MD, VA, TN, AR, WV, KY, NC, SC, AL, MS, LA, GA, FL) 82

SW Mountain (MT, ID, UT, WY, AZ, CO, NM, OK, TX) 163

West (AK, HI, NV, WA, OR, CA) 127

Foreign 156

$\begin{array}{lr}\text { LASL } & 197\end{array}$

$\begin{array}{ll}\text { TOTAL } & 1002\end{array}$

5. Fields of Interest

Nuclear and Particle Physics 947

Nuclear Chemistry 144

Solid State Physics \& Materials Science 252

$\begin{array}{ll}\text { Theory } & 163\end{array}$

Biomedical and Biological Applications 448

$\begin{array}{ll}\text { Isotope Production } & 101\end{array}$

Weapons Neutron Research $\quad 87$

$\begin{array}{lc}\text { Data Acquisition } & 156\end{array}$

Administration, Coordination, Facilities, Operations,

Practical Applications, etc.

166

(Note: These numbers do not add to total membership because of multiple interests.) 


\section{APPENDIX}

\section{RESEARCH GUESTS AT LAMPF DURING PERIOD AUGUST 1, 1977 - OCTOBER 31, 1977}

Gary S. Adams ............ UC, Los Angeles Kirk P. Allbright .............Univ. of Texas John C. Allied ............. Univ. of Houston Rosemary R. Altemus .........Univ. of Virginia James Amann ........ Carnegie-Mellon Univ. Herbert Anderson ........... Univ. of Chicago L. B. Auerbach .............. Temple Univ. Helmut W. Baer ... Case Western Reserve Univ. Howard 1. Balshem ............ Temple Univ. Thomas S. Bauer ........... UC, Los Angeles Philip R. Bevington Case Western Reserve Univ. Tarlochan S. Bhatia ........ Texas A\&M Univ C. W. Bjork ............ Univ. of Wyoming Gary S. Blanpied .............Univ. of Texas Carolyn A. Blie; ......... UNM Cancer Center Felix H. Boehm ....... California Inst. of Tech. Paul R. Bolton ................. Yale Univ. C. A. Bordner ............... Colorado College Jonathan S. Boswell .......... Univ. of Vircinia Richard L. Boudrie .......... Univ. of Colorado Kenneth Boyer............... Univ. of Texas W. J. Braithwaite .............Univ. of Texas Ivar G. Brissaud . Institut de Physique Nucleaire H. C. Bryant ............Univ. of New Mexico James A. Buchanan .............. Rice Univ. Tony J. Buchen ........... Univ. of New Mexico G. R. Burleson ........ New Mexico State Univ. Jeanne L. Burmester ...... UNM Cancer Center Elizabeth Cameron ........ UNM Cancer Center Roger D. Carlini ...........Univ. of New Mexic Rebecca Carrington........... Stanford Univ. David T. Chiang .......... Univ. of Washington Larry B. Church ............. Reed College John M. Clement ............... Rice Univ. Robert K. Cole ..... Univ. of Southern California William Cottingame ............Univ. of Texas C. Alton Coulter ............ Univ. of Alabama Frank H. Cverna ... Case Western Reserve Univ. Steven H. Dam ........ Univ. of South Carolina Dietrich Dehnhard ........ Univ. of Minnesota Judith M. Dembo ........ UNM Cancer Center William F. Denig ........... Utah State Univ. Arthur B. Denison ......... Univ. of Wyoming Virginia L. Desjardin ...... UNM Cancer Center
Michael J. Devereux... New Mexico State Univ. Satish Dhawan ................. Yale Univ. N. J. DiGiacomo ........... Univ. of Colorado Byron Dieterle ............Univ. of New Mexico Joey B. Donahue .......... Univ. of New Mexico Hermann J. Donnert ....... Kansas State Univ. Alexander Doron ............. Tel-Aviv Univ. Mohan Doss .......... Carnegie-Mellon Univ. Jean Duclos ............... C.E.N., Saclay Minh Duong-Van. Stanford Linear Accel. Center Patrick O. Egan ................. Yale Univ. Ralph A. Eichler ............ Stanford Univ. Diane E. Ewing ....... New Mexico State Univ. Donald R. Fenstermacher .. UNM Cancer Center Herman Feshbach . . Massachusetts Inst. of Tech. John C. Fong .............. UC, Los Angeles Dan R. Fortney .............. Purdue Univ. Sherman Frankel ........ Univ. of Pennsylvania John S. Fraser ...... Chalk River Nuclear Labs. William Frati .......... Univ. of Pennsylvania Hans Frauenfelder............ Univ. of Illinois M. Magdy Gazzaly ....... Univ. of Pennsylvania David R. Giebink .............Univ. of Texas Shalev Gilad ............... Tel-Aviv Univ. Charles Giashausser ........... Rutgers Univ. George Glass ............. Texas A\&M Univ. Roy J. Glauber ................ Harvard Univ. Robert L. Gluckstern ....... Univ. of Maryland Robert M. Gougelet ....... UNM Cancer Center Charles A. Goulding ........ Florida A\&M Univ. Charles M. Grassl .......... Univ, of Wyoming Steven J. Greene ................Univ. of Texas Manuel G. Gutierrez ......... Stanford Univ. Peter K. Haff . ............... Yale Univ. Klaus Halbach ........ Lawrence Berkeley Lab. Robert W. Hamm ......... Texas A\&M Univ. Arthur D. Hancock .......... Univ. of Houston William J. Hanna ............ Univ. of Colorado Herbert E. Henrikson . . . California Inst. of Tech. Constance C. Her .......... Northwestern Univ. John C. Hiebert ........... Texas A\&M Univ. Virgil L. Highland ............ Temple Univ. John C. Hill ................ Iowa State Univ. Nathaniel W. Hill ..... Oak Ridge National Lab. 
John F. Hills ... Univ. of Colorado Med. Center Norton M. Hintz ......... Univ. of Minnesota William .J. Hoffert ............. Indiana Univ. John H. Hoftiezer . .............. Rice Univ. Kenneth R. Hogstrom .... UNM Cancer Center Charles L. Hollas ..............Univ. of Texas Barrie E. Hughes ............ Stanford Univ. Vernon $W$. Hughes .............. Yale Univ. George J. Igo ............. UC, Los Angeles Hikaru Inoue .............. Hiroshima Univ. Harvey Israel ............ Private Consultant Steven G. Iversen ......... Northwestern Univ. Mark J. Jakobson ........... Univ, of Montana R. H. Jeppesen ............ Univ. of Montana K. F. Johnson ............. Temple Univ. William Johnson ........... Univ. of Rochester David M. Judd ................ Rice Univ. Mark O. Kaletka .......... Northwestern Univ. Heinz F. Kaspar .................. SIN Brad D. Keister ........ Carnegie-Mellon Univ. Charles A. Kelsey ......... UNM Cancer Center Charles R. Key .........UNM Sch. of Medicine W. Wayne Kinnison .......... Univ. of Chicago H. B. Knowles ........ Washington State Univ. Bonnie L. Kober ......... UNM Cancer Center Thomas Kozlowski ... Brookhaven National Lab. J. J. Krushaar ............. Univ. of Colorado Ray Kunselman ........... Univ. of Wyoming Gary Kyle .............. Univ. of Minnesota John A. Lake ........... UNM Cancer Center Richard G. Lane ......... UNM Cancer Center Chris P. Leavitt ........... Univ. of New Mexico Bonnie A. Leonard ........ UNM Cancer Center Albert P. Li ........... UNM Cancer Center Bernard J. Lieb .......... George Mason Univ. Rodger Liljestrand............Univ. of Texas V. Gordon Lind ............ Utah State Univ. Jerry E. Lisantti ............. Univ. of Idaho Stanley Livingston .......... Private Consultant Joseph W. Lo ............. Univ. of Houston Earl L. Lomon .... Massachusetts Inst. of Tech. Daniel C. $\mathrm{Lu}$................. Yale Univ. Steven C. Luckstead ... Washington State Univ. William G. Lynch ........ Univ. of Washington D. J. Malbrough ....... Univ. of South Carolina D. Mark Manley .......... Univ. of Wyoming Fesseha Mariam ................ Yale Univ. Howard S. Matis ............ Univ. of Chicago Robert E. McAdams ......... Utah State Univ. James S. McCarthy ...........Univ. of Virginia
W. K. McFarlane ............. Temple Univ. Dorothy H. McGrath ...... UNM Cancer Center M. McNaughton ... Case Western Reserve Univ. Thomas P. McPharlin ......... Stanford Liniv. Katharine Metropolis ...... UNM Cancer Center Ralph C. Minehart ...........Univ. of Virginia Murray Moinester ............ Tel-Aviv Univ. C. Fred Moore ................ Univ, of Texas Urs F. Moser .................... Yale Univ. Olive D. Moss .......... UNM Cancer Center Donald W. Mueller .......... Private Consultant Gordon S. Mutchler .............. Rice Univ. Hermann Nann .......... Northwestern Univ. Robert A. Naumann ............Princeton Univ. Charles Newsom ..............Univ. of Texas L. C. Northcliffe ........... Texas A\&M Univ. Andrew W. Obst .......... Northwestern Univ. Alana M. Olcott ......... UNM Cancer Center Michael A. Oothoudt ....... Univ, of Minnesota Lewis J. Orphanos ...........Univ, of Virginia Sivarajan Pandiar............. Purdue Univ. Jeff W. Parrish ............ Univ. of Oregon Gianni Pauletta ............. UC, Los Angeles Gregory P. Pepin ............... Rice Univ. R. J. Peterson ............ Univ. of Colorado Rohert F. Petry ............Univ. of Oklahoma Lawrence S. Pinsky ......... Univ. of Houston Hans S. Plendl ............ Florida State Univ. Norbert T. Porile ... . . . . . . . . . . Purdue Univ. Richard .J. Powers ...... California Inst. of Tech. Barry M. Preedom ..... Univ. of South Carolina Clifford Qualls ............Univ. of New Mexico R. D. Ransome ............... Univ. of Texas Glen A. Rebka ........... Univ. of Wyoming Peter J. Riley .................Univ. of Texas Robert A. Ristinen .......... Univ. of Colorado Janet K. Robinson ........ UNM Cancer Center Isaac I. Rosen ........... UNM Cancer Center Ted D. Rupp ............ Univ. of New Mexico Norio Saito ........ Central Res. Lab. of Hitachi Jose M. Sala ............ UNM Cancer Center Mark A. Schardt .... ....... Asínona State Univ. Gerhard Schmidt ............Princeton Univ. Paul J. Schmit ............ Northwestern Univ. Stan O. Schriber .... Chalk River Nuclear Labs. K. A. Schumacher.... Brookhaven National Lab. Kim I. Scott ............ UNM Cancer Center Ralph E. Segel ........... Northwestern Univ. Kamal K. Seth ......... Norrthwestern Univ. Hasan Sharifian .......... Univ. of New Mexico 
Joseph D. Sherman .... Carnegie-Mellon Univ. D. G. Shirk ................ Iowa State Univ. Frank T. Shively ....... Lawrence Berkeley Lab. Steven L. Simon .......... UNM Cancer Center Roy T. Slice ............ UNM Cancer Center Alfred R. Smith .......... UNM Cancer Center Gregory Smith ............ Univ. of Colorado Lester E. Smith ..............Univ. of Texas John W. Somers ......... UNM Cancer Center Larry B. Sorensen ............ Univ, of Illinois Paul Souder ................. Yale Univ. Jan Soukup .............. . UC, Los Angeles Robert L. Stearns ............ Vassar College Rolf M. Steffen ............... Purdue Univ. Charles Sternhagen ....... UNM Cancer Center L. Wayne Swenson ........ Oregon State Univ. M. Takai ................ Hamamatsu Univ. Fran L. Talley ........... UNM Cancer Center Yoshiaki Tanaka ................................ Univ. Robert L. Tanner ........... Private Consultant Charles A. Taylor ....... Norfolk State College Andrew M. Thomson ...... Univ. of Minnesota James R. Tinsley ............. Univ. of Oregon Jean A. Tolmas .......... UNM Cancer Center Hossein Tootoonchi ....... Univ. of New Mexico Gregg S. Tracton ........ Univ, of Pennsylvania Christopher Tschalar ................ SIN J. K. Valentine .............. Temple Univ. Ellen J. Vandergust ....... UNM Cancer Center Philip Varghese ............. Univ. of Oregon Stephen L. Verbeck ... New Mexico State Univ. Jurgen Vetter ......... Universitat Heidelberg
( . F. Von Essen ................ SIN Wolfram Von Witsch ............ Rice Univ. Jean-Luc Vuilleumier .. California Inst. of Tech. E. A. Wadlinger .............. Univ. of Virginia John B. Walter .......... Univ. of Wyoming Angel T. M. Wang ........ SUNY, Stony Brook Keh-Chung Wang ...... California Inst. of Tech. K. H. Wang ................ Baylor Univ. Linda .J. Warnes ......... UNM Cancer Center Mario Weiss ......................... NP Joseph Weneser ...... Brookhaven National Lab. W. R. Wharton ........ Carnegie-Mellon Univ. Charles A. Whitten .......... UC, Los Angeles Harvey B. Willard.. Case V'estern Reserve U'iniv. Thomas M. Williams ........ Univ. of Hoiston Stzanne Willis ................. Yale Univ. Henry Willmes ................ Univ. of Idaho Stephany Wilson ........ UNM Cancer Center Eric Winkelmann .. Case Western Reserve Univ. David Wolfe ............. Univ. of New Mexico Bernard E. Wood ........... Univ. of Oregon H. G. Worstell ............ Private Consultant Abdul-Halim A. Wriekat ....... UC, Los Angeles Courtenay S. Wright ......... Univ. of Chicago Deane Yang .......... Univ. of Pennsylvania Vincent Yuan ............... Univ. of Illinois .John M. Yuhas .......... UNM Cancer Center Benjamin Zeidman ...... Argonne National Lab. Hans J. Ziock ............... Univ of Virginia Klaus O. H. Ziock ...........Univ. of Virginia Klaus P. Ziock .............. Univ. of Virginia 